\title{
Distribution of East Eurasian Y-Chromosome and Mitochondrial DNA Haplogroups across Eurasia: Insights into the Genetic Ancestry of Bulgarians
}

\author{
Sena Karachanak-Yankova ${ }^{1^{*}}$, Desislava Nesheva ${ }^{1 *}$, Angel S. Galabov², Draga Toncheva ${ }^{1 \#}$ \\ ${ }^{1}$ Department of Medical Genetics, Medical University of Sofia, Sofia, Bulgaria \\ ${ }^{2}$ The Stephan Angeloff Institute of Microbiology, Bulgarian Academy of Sciences, Sofia, Bulgaria \\ Email: \#dragatoncheva@gmail.com
}

Received 18 August 2015; accepted 13 October 2015; published 16 October 2015

Copyright (C) 2015 by authors and Scientific Research Publishing Inc.

This work is licensed under the Creative Commons Attribution International License (CC BY). http://creativecommons.org/licenses/by/4.0/

(c) (7) Open Access

\section{Abstract}

The modern Bulgarian mitochondrial DNA (mtDNA) and Y-chromosome gene pools predominantly consist of Western Eurasian haplogroups. In contrast, the Eastern Eurasian lineages are found at very low frequencies in Bulgarians, being represented only by mtDNA haplogroups $C(0.2 \%), D$ (0.4\%) and Z (0.1\%) (Karachanak et al., 2012) and Y-chromosome haplogroups $\mathrm{C}, \mathrm{N}$ and $\mathrm{Q}$ (each 0.5\%) (Karachanak et al., 2013). A similar pattern is observed in ancient mtDNA samples of proto-Bulgarian human remains, which belong exclusively to Western Eurasian mtDNA haplogroups (Nesheva et al., 2015). In order to investigate Bulgarian ancestry from the perspective of Eastern Eurasian haplogroups, we have analyzed the distribution of $\mathrm{Y}$-chromosome haplogroups $\mathrm{C}, \mathrm{N}$ and $Q$ and mtDNA haplogroups $C, D$ and $Z$ across Eurasia. The survey was performed using literature data for more than 15,000 individuals from different Eurasian (sub-) populations for each of these haplogroups. The collected data were used to construct Eurasian frequency maps of the considered haplogroups and to test the significance of their incidence between Bulgarians and Europeans, European neighboring populations of Bulgaria and populations, which according to some historical conceptions could have common ancestry with proto-Bulgarians, namely: Altaian, Caucasus, Siberian and Central Asian populations. The spatial distribution of mtDNA haplogroups C, $D$ and $\mathrm{Z}$ and $\mathrm{Y}$-chromosome haplogroups $\mathrm{C}, \mathrm{N}$ and $\mathrm{Q}$ contrasts their high frequency among Altaic populations and their occasional appearance in Bulgarians. Furthermore, the comparison of the occurrence of these haplogroups shows no link between Bulgarians and Altaic and Caucasus populations. Based on the substantial genetic input of proto-Bulgarians to the modern Bulgarian gene

\footnotetext{
*These authors have equally contributed to this work.

${ }^{\#}$ Corresponding author.
}

How to cite this paper: Karachanak-Yankova, S., Nesheva, D., Galabov, A. S., \& Toncheva, D. (2015). Distribution of East Eurasian Y-Chromosome and Mitochondrial DNA Haplogroups across Eurasia: Insights into the Genetic Ancestry of Bulgarians. Advances in Anthropology, 5, 205-266. http://dx.doi.org/10.4236/aa.2015.54019 
pool, the present study confirms the nonexistence of a close Y-chromosomal or mtDNA link between proto-Bulgarians on the one hand and Altaic and Caucasus populations on the other.

\title{
Keywords
}

\author{
Bulgarians, Y-Chromosome, mtDNA, Haplogroup
}

\section{Introduction}

The territory of Bulgaria has been involved in different migration routes ever since the modern human settlement of Europe in the Upper Paleolithic. Later, it has served as a refugium and fuelled different expansion routes of postglacial re-colonization. The area of present-day Bulgaria also played a significant role in the Neolithic spread (Calafell et al., 1996; Nitecki \& Nitecki, 2013; Ray \& Adams, 2001). This prehistorical population background was overlaid by the inhabitance of the Thracians, and later by the invasions of Slavs and proto-Bulgarians, which were considered as populations playing important role in the modern Bulgarian ethnogenesis (Dobrev, 1994, 2005; Haefs, 2009).

The most disputable issue of the Bulgarian past is the origin of proto-Bulgarians. Their ancestry has been widely debated, as there are even theories describing them as Altaic and Turko-Mongolic people (Zlatarski, 1970). According to novel archeological, historical and linguistic evidence, the ancient (proto-) Bulgarians made a substantial contribution to the modern Bulgarian gene pool (Dimitrov, 2005; Rashev, 1993).

The previously performed phylogenetic analysis of 13 ancient mtDNA samples retrieved from proto-Bulgarian human remains and discovered in three necropolises in Bulgaria dating to VIII-X century AD identified mtDNA haplogroups, which were found in present-day European and Western Eurasian populations. These results pointed to a Western Eurasian matrilineal origin for proto-Bulgarians as well as to a genetic similarity between them and modern Bulgarians (Nesheva et al., 2015).

The high-resolution phylogenetic analyses of Y-chromosome and mitochondrial DNA (mtDNA) diversity of modern Bulgarians in samples comprising 808 and 855 subjects, respectively, showed that their gene pool was predominantly composed by Western Eurasian haplogroups. On the other hand, Eastern Eurasian haplogroups are represented at negligible frequencies. Thus, mtDNA haplogroups $\mathrm{C}, \mathrm{D}$ and $\mathrm{Z}$ account for $0.2 \%, 0.4 \%$ and $0.1 \%$ of the modern Bulgarian gene pool, respectively and Y-chromosome haplogroups $\mathrm{C}, \mathrm{N}$ and $\mathrm{Q}$ are each at frequency of $0.5 \%$. The interpopulational comparisons of Y-chromosome and mtDNA haplogroup frequencies locate modern Bulgarians among European populations in both cases (Karachanak et al., 2012, 2013).

In order to further test the reliability of the theories of Bulgarian, and in particular of proto-Bulgarian origin, we have depicted the frequency gradients of $\mathrm{Y}$-chromosome haplogroups $\mathrm{C}, \mathrm{N}$ and $\mathrm{Q}$ and mtDNA haplogroups $\mathrm{C}, \mathrm{D}$ and $\mathrm{Z}$ across Eurasia and we have tested the significance of their incidence between Bulgarians and Europeans, European neighboring populations of Bulgaria and populations, which according to some historical conceptions could have common ancestry with proto-Bulgarians, namely: Altaian, Caucasus, Siberian and Central Asian populations.

\section{Materials and Methods}

We have collected literature data for the frequencies of $\mathrm{Y}$-chromosome haplogroups $\mathrm{C}, \mathrm{N}$ and $\mathrm{Q}$ and $\mathrm{mtDNA}$ haplogroups $\mathrm{C}, \mathrm{D}$ and $\mathrm{Z}$ in Eurasian populations. The gathered data for the Y-chromosome haplogroups comprise 19856 samples from 186 (sub-) populations for $\mathrm{Hg} \mathrm{C}, 16418$ from 173 (sub-) populations for $\mathrm{Hg} \mathrm{N}$ and 15652 samples from 160 (sub-) populations for Hg Q (supplementary Tables S1(a)-(c), respectively). For the mtDNA haplogroups we have collected data for 35083 samples representing 226 (sub-) populations for $\mathrm{Hg} \mathrm{C}$, 38039 from 242 (sub-) populations for haplogroup D and 17716 samples from 160 (sub-) populations for $\mathrm{Hg} \mathrm{Z}$ (supplementary Tables S2(a)-(c), respectively). These data were used to construct frequency maps by using the Surfer Golden software following the Kriging procedure.

The occurrences of the aforementioned haplogroups were compared between modern Bulgarians and: Europeans, European neighboring populations of Bulgaria, Altaian, Caucasus, Siberian and Central Asian populations. The comparison was performed through a chi-square test using XLSTAT. 


\section{Results}

The frequency maps of Y-chromosome haplogroups $\mathrm{C}, \mathrm{N}$ and $\mathrm{Q}$ are given in Figure 1.

Haplogroup $\mathrm{C}$, one of the earliest Out-of-Africa founder types, has highest frequencies in the middle latitudes of Asia. It has overwhelming presence among: Oroqens (90.9\%), Evens (74.2\%), Evenks (68.4\%) (Hammer et al., 2005), Buryats (68.2\%), Mongols (65.2\%) and Kalmyks (62.6\%) (Malyarchuk et al., 2010). The frequency of haplogroup $\mathrm{C}$ decreases in South Asia and diminishes in Europe with only few representatives in the south of the continent.

The frequency map of $\mathrm{Hg} \mathrm{N}$, which is considered of Southern East Asia origin (Shi et al., 2013), depicts the prevalence of this haplogroup mainly in Northern Eurasia. Thus, it is most numerous in European Russia, in particular in: Kursk (92.5\%) (Mirabal et al., 2009), Tver (78.9\%), Mezen (53.7\%), Krasnoborsk (39.6\%), Pinega (39.5\%) and Vologda (38.8\%); in Siberia, among Yakuts (84.2\%) (Derenko et al., 2007a; Sengupta et al., 2006) and Khanty (81.5\%) (Mirabal et al., 2009); and among Swedish Saami (44.7\%) (Karlsson et al., 2006). Haplogroup $\mathrm{N}$ abruptly dissipates in lower latitudes, being virtually absent in southern Eurasia, with the exception of certain southwest Chinese ethnic groups such as Sichuan (13.2\%) and Yunnan (10.7\%) (Zhong et al., 2011). Unlike the other considered haplogroups, $\mathrm{Hg} \mathrm{N}$ has quite uneven distribution among European populations which is summarized in Table 1. It is apparent that $\mathrm{Hg} \mathrm{N}$ is most prominent in East Slavic populations (Russians, Belarusians and Ukrainians) (Battaglia et al., 2009; Kushniarevich et al., 2013; Mirabal et al., 2009) and Swedish Saami (Karlsson et al., 2006), but is almost lacking among other Europeans.

Despite its Asian origin (Sharma et al., 2007), Hg Q is a minor Y-chromosome haplogroup in Eurasia having two occasional frequency peaks in Northwest Siberia among Kets and Selkups (93.8\% and 66.4\%, respectively) (Karafet et al., 2002) and in Altai people (17.4\%) (Hammer et al., 2005), as its frequency steeply declines outside these regions.

The spatial frequency maps of mtDNA haplogroups C, D and Z are presented in Figure 2.

Haplogroups C and D, which have evolved in Eastern Asia and expanded after the Last Glacial Maximum, comprise almost half of the northern and eastern Asian mtDNA gene pool (Derenko et al., 2007b; Derenko et al., 2010).

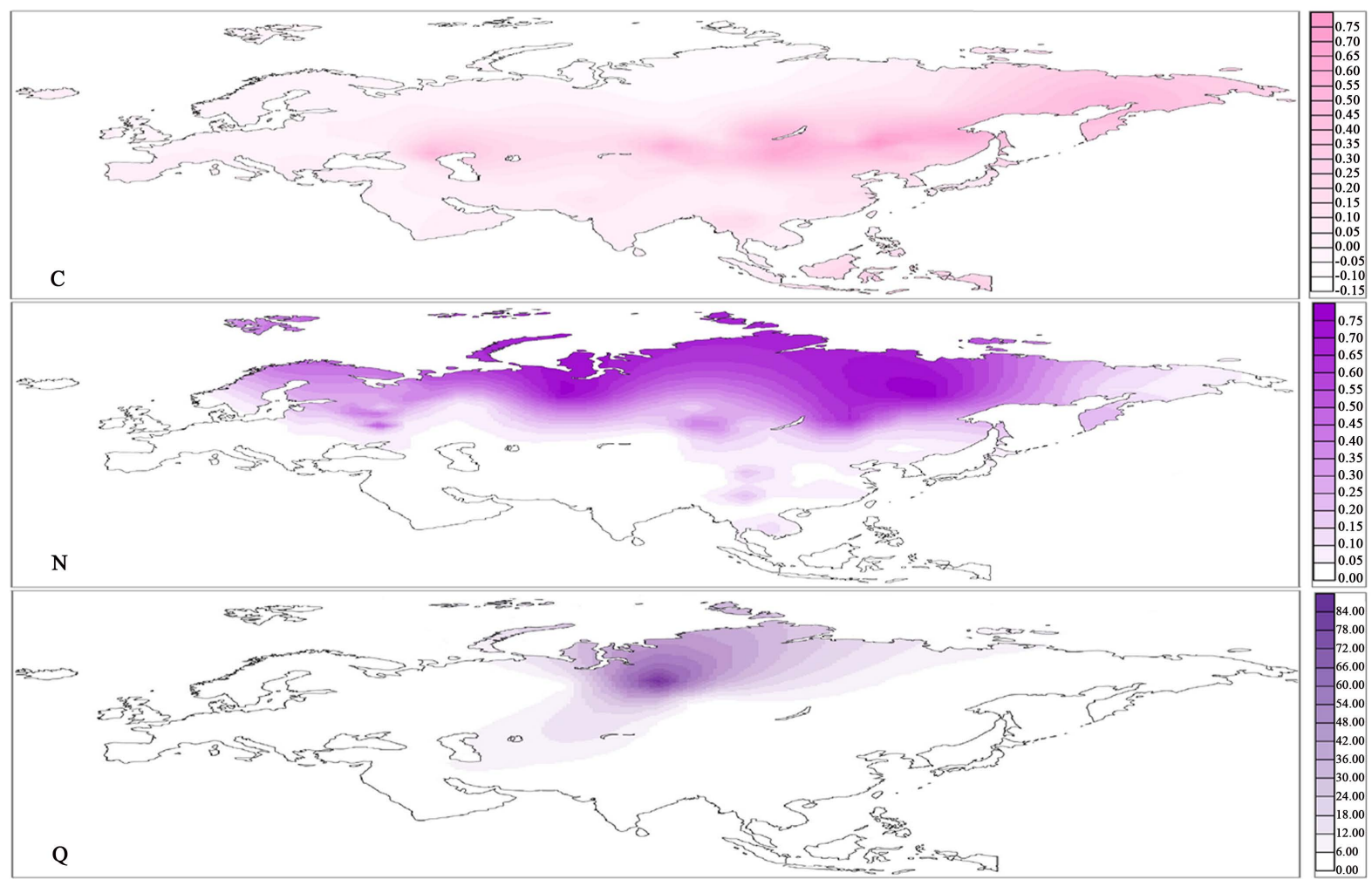

Figure 1. Eurasian frequency distributions of Y-chromosome haplogroups C, N and Q. 
Table 1. Percent frequency of Y-chromosome haplogroup N in European populations. N-number of analyzed individuals.

\begin{tabular}{|c|c|c|c|}
\hline Populations & \multirow[t]{2}{*}{$\mathrm{N}$} & \multirow[t]{2}{*}{$\mathrm{Hg} \mathrm{N} \%$} & \multirow[t]{2}{*}{ Reference } \\
\hline European Russia, Belorussia and Ukrain & & & \\
\hline - Belarussian-East Polesie & 96 & 8.3 & Kushniarevich et al., 2013 \\
\hline - Belarussian-West Polesie & 121 & 8.3 & Kushniarevich et al., 2013 \\
\hline - Belarussian-Centre & 88 & 8 & Kushniarevich et al., 2013 \\
\hline - Belarussian-East & 86 & 7 & Kushniarevich et al., 2013 \\
\hline - Belarussian-North & 101 & 15.8 & Kushniarevich et al., 2013 \\
\hline - Belarussian-West & 73 & 13.7 & Kushniarevich et al., 2013 \\
\hline - Russia-Arkhangelski & 28 & 28.6 & Mirabal et al., 2009 \\
\hline - Russia-Belgorod & 143 & 12.6 & Battaglia et al., 2009 \\
\hline - Russia-Belgorod & 143 & 12.6 & Battaglia et al., 2009 \\
\hline - Russia-Kashin & 73 & 11 & Battaglia et al., 2009 \\
\hline - Russia-Krasnoborsk & 91 & 39.6 & Battaglia et al., 2009 \\
\hline - Russia-Kuban Cossacs & 90 & 7.8 & Battaglia et al., 2009 \\
\hline - Russia-Kursk & 40 & 92.5 & Mirabal et al., 2009 \\
\hline - Russia-Livni & 110 & 5.5 & Battaglia et al., 2009 \\
\hline - Russia-Livni & 110 & 5.5 & Battaglia et al., 2009 \\
\hline - Russia-Mezen & 54 & 53.7 & Battaglia et al., 2009 \\
\hline - Russia-Ostrov & 75 & 28 & Battaglia et al., 2009 \\
\hline - Russia-Pinega & 114 & 39.5 & Battaglia et al., 2009 \\
\hline - Russia-Porhov & 57 & 19.3 & Battaglia et al., 2009 \\
\hline - Russia-Pristen & 45 & 13.3 & Battaglia et al., 2009 \\
\hline - Russia-Pskov & 132 & 24.2 & Battaglia et al., 2009 \\
\hline - Russia-Repievka & 96 & 6.3 & Battaglia et al., 2009 \\
\hline - Russia-Roslavl & 107 & 14 & Battaglia et al., 2009 \\
\hline - Russia-Tver & 38 & 78.9 & Mirabal et al., 2009 \\
\hline - Russia-Unzha & 52 & 13.5 & Battaglia et al., 2009 \\
\hline - Russia-Unzha & 52 & 13.5 & Battaglia et al., 2009 \\
\hline - Russia-Vologda & 121 & 38.8 & Battaglia et al., 2009 \\
\hline - Ukraine & 92 & 6.5 & Battaglia et al., 2009 \\
\hline \multicolumn{4}{|l|}{ Central Europe } \\
\hline - Czechs & 75 & 2.7 & Battaglia et al., 2009 \\
\hline - Germany & 16 & 0 & Battaglia et al., 2009 \\
\hline - Hungary & 53 & 0 & Battaglia et al., 2009 \\
\hline - Poland & 99 & 1 & Battaglia et al., 2009 \\
\hline \multicolumn{4}{|l|}{ Western Europe } \\
\hline - Ac,ores & 121 & 0 & Capelli et al., 2007 \\
\hline - Antwerpen & 72 & 0 & Capelli et al., 2007 \\
\hline - Corsica & 34 & 0 & Derenko et al., 2007a \\
\hline - France & 23 & 0 & Battaglia et al., 2009 \\
\hline - Gipuzkoa Basques & 74 & 0 & Kushniarevich et al., 2013 \\
\hline - Italy-Central Marche & 59 & 0 & Larmuseau et al., 2011 \\
\hline - Italy-Elba Island Tuscany & 95 & 0 & Larmuseau et al., 2011 \\
\hline
\end{tabular}


S. Karachanak-Yankova et al.

\section{Continued}

- Italy-West Calabria

- Kempen Belgium

- Madeira

- Mechelen Belgium

- Noord-Brabant Netherlands

- Norway

- Portugal-Central

- Portugal-North

- Portugal-South

- Sardinia

- Sicily

- Spain-Alto Urgel

- Spain-Cerdãna

- Spain-Cinco Villas

- Spain-Jacetania

- Spain-Vallede Ar'an

- Swedish Saami

- Vlaams and Waals Brabant Belgium

$\begin{array}{ccc}57 & 0 & \text { Larmuseau et al., 2011 } \\ 77 & 1.3 & \text { Capelli et al., 2003 } \\ 129 & 2.3 & \text { Gonçalves et al., 2005 } \\ 63 & 3.2 & \text { Capelli et al., 2003 } \\ 130 & 0 & \text { Capelli et al., 2003 } \\ 201 & 1 & \text { Semino et al., 2000 } \\ 102 & 0 & \text { Capelli et al., 2007 } \\ 101 & 0 & \text { Capelli et al., 2007 } \\ 100 & 0 & \text { Capelli et al., 2007 } \\ 78 & 0 & \text { Francalacci et al., 2003 } \\ 51 & 0 & \text { Francalacci et al., 2003 } \\ 34 & 0 & \text { López-Parra et al., 2009 } \\ 37 & 0 & \text { López-Parra et al., 2009 } \\ 42 & 0 & \text { López-Parra et al., 2009 } \\ 31 & 0 & \text { López-Parra et al., 2009 } \\ 25 & 0 & \text { López-Parra et al., 2009 } \\ 38 & 44.7 & \text { Karlsson et al., 2006 } \\ 135 & 0.7 & \text { Larmuseau et al., 2011 }\end{array}$

Southeastern Europe

- Albania

55

- Bosniacs

\begin{tabular}{|c|c|c|}
\hline & 0 & Battaglia et al., 2009 \\
\hline & 0 & Battaglia et al., 2009 \\
\hline & 0 & Battaglia et al., 2009 \\
\hline & 6.2 & Battaglia et al., 2009 \\
\hline & 0.5 & Karachanak et al., 2013 \\
\hline & 0 & Martinez et al., 2007 \\
\hline & 0 & Martinez et al., 2007 \\
\hline & 0 & Martinez et al., 2007 \\
\hline & 2.2 & Battaglia et al., 2009 \\
\hline & 0 & Battaglia et al., 2009 \\
\hline & 0.5 & Noveski et al., 2010 \\
\hline & 0 & Battaglia et al., 2009 \\
\hline & 0 & King et al., 2008 \\
\hline & 0 & King et al., 2008 \\
\hline & 0 & King et al., 2008 \\
\hline 7 & 0 & Battaglia et al., 2009 \\
\hline & 1.6 & Varzari et al., 2013 \\
\hline & 0.7 & Martinez-Cruz et al., 2012 \\
\hline & 1.9 & Regueiro et al., 2012 \\
\hline & 0 & Battaglia et al., 2009 \\
\hline
\end{tabular}

- Bosnian Croats

- Bosnian Serbs

- Bulgaria

- Crete-Heraklion Prefecture

- Crete-Lasithi Plateau

- Crete-Lasithi Prefecture

- Croats

- FYROM

- Greece-Crete

\section{7}

- Macedonian Greeks

- Moldavia

- Serbia

- Slovenia 


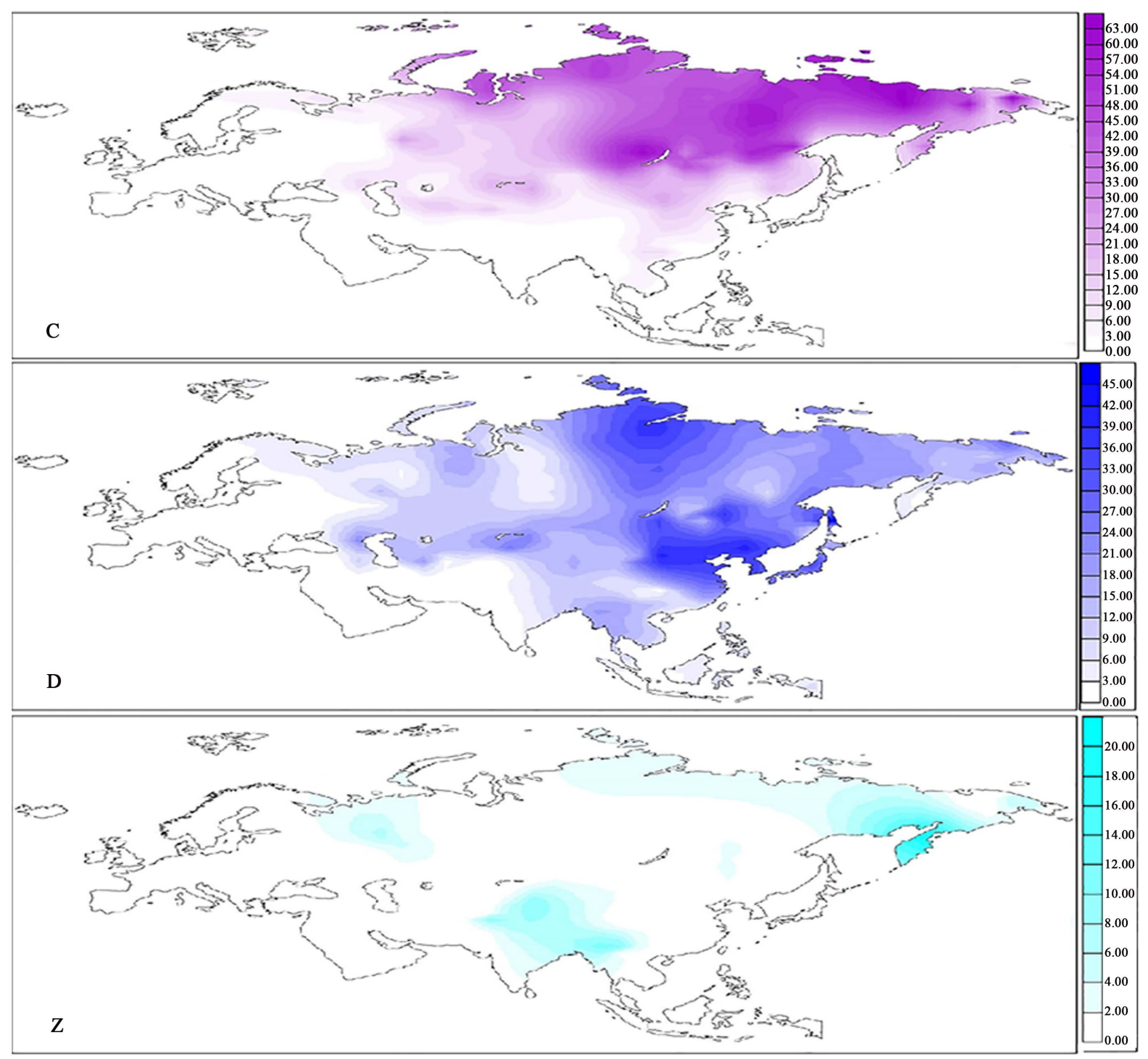

Figure 2. Eurasian frequency distributions of mtDNA haplogroups C, D and Z.

Haplogroup $\mathrm{C}$ is prevalent in Siberia. It has highest frequencies in regions inhabited by Yukaghirs (up to 72.2\%) (Volodko et al., 2008), Evenks (up to 71.8\%) and Tofalars (up to 62.1\%) (Derenko et al., 2010). The occurrence of haplogroup C decreases to $5 \%$ towards the Volga-Ural region, Central Asia and the Indian subcontinent; which together delineate the diminishing border of the haplogroup.

The distributions of haplogroups $\mathrm{C}$ and $\mathrm{D}$ are almost overlapping. They slightly differ in haplogroup $\mathrm{D}$ frequency maximums which are found in: Oroks of Sakhalin (68.9\%), Sojots from the Baikal region (46.7\%), Orochens (43.2\%), Manchus (42.6\%) (Zhao et al., 2011) and Dolgans in North Siberia (39.5\%) (Derenko et al., 2010). Furthermore, the frequency of $\mathrm{Hg} \mathrm{D}$ drops below 5\% more westward and southward than $\mathrm{Hg} \mathrm{C}$, but it is also nearly inexistent in Europe and West Asia.

The minor mtDNA haplogroup Z, which is of Central Asian origin, has only a few frequency foci in Eurasia. They are scattered in: Northeastern Asia in Koryaks (21.7\%); in the Volga-Ural region in Udmurts (16.7\%) (Grosheva et al., 2014) and in parts of Central South Asia among Nu (16.7\%) (Liu et al., 2011), Hazara (13\%) (Quintana-Murci et al., 2004), and Kazakhs (11.3\%) (Yao et al., 2004).

The results of the chi-square test of the occurrence of Y-chromosome haplogroups $\mathrm{C}, \mathrm{N}$ and Q (supplementary Table S3) and mtDNA haplogroups C and D (supplementary Table S4) are presented in Table 2 and Table 3, respectively. 
Table 2. Comparison of the incidence of Y-chromosome haplogroups $\mathrm{C}, \mathrm{N}$ and $\mathrm{Q}$ between Bulgarians and different population groups.

\begin{tabular}{ccccccc}
\hline Population group & N & Hg C & Hg N & Hg Q & $p^{* *}$ \\
\hline Altaian populations & 465 & 122 & 45 & 68 & $<0.0001$ \\
Siberian populations & 842 & 257 & 478 & 9 & $<0.00001$ \\
Central Asian populations & 700 & 67 & 111 & 49 & $<0.00001$ \\
European populations & 2617 & 4 & 77 & 3 & 0.000035 \\
Bulgarians' European neighbouring populations & 520 & 0 & 4 & 1 & 0.289988 \\
Bulgarians & 808 & 4 & 4 & 4 & \\
\hline
\end{tabular}

N-number of samples analyzed; "The populations consisting the population groups and their references are given in supplementary Table S3; p-value population group $v s$ Bulgarians.

Table 3. Comparison of the incidence of mtDNA haplogroups $\mathrm{C}$ and D between Bulgarians and different population groups.

\begin{tabular}{ccccc}
\hline Population group $^{*}$ & $\mathrm{~N}$ & $\mathrm{Hg} \mathrm{C}$ & $\mathrm{Hg} \mathrm{D}$ & $p^{* *}$ \\
\hline Altaian populations & 1058 & 252 & 223 & $<0.0001$ \\
Siberian populations & 2892 & 911 & 570 & $<0.0001$ \\
Central Asian populations & 3078 & 314 & 444 & $<0.0001$ \\
Caucasus populations & 829 & 92 & 54 & 0.00001 \\
European populations & 8229 & 20 & 21 & 0.873002 \\
Bulgarians' European neighbouring populations & 658 & 1 & 1 & 3 \\
Bulgarians & 855 & 2 & 3
\end{tabular}

$\mathrm{N}$-number of samples analyzed; "The populations consisting the population groups and their references are given in supplementary Table S4; ${ }^{* *} p$-value population group $v s$ Bulgarians.

The occurrence of Y-chromosome haplogroups $\mathrm{C}, \mathrm{N}$ and $\mathrm{Q}$ shows that there is statistically significant difference between Bulgarians and: Altaian, Siberian, Central Asian and European populations $(p<0.0001, p<$ $0.00001, p<0.00001$ and $p=0.000035$, respectively). Furthermore, the chi-square test shows a Y-chromosome relation between Bulgarians and their European neighboring populations ( $p=0.289988)$.

The absolute frequencies of mtDNA haplogroups $\mathrm{C}$ and $\mathrm{D}(\mathrm{Hg} \mathrm{Z}$ was excluded due to lack of data for part of the populations) point that there is significant difference between Bulgarians and: Altaian, Siberian, Central Asian and Caucasus populations ( $p<0.0001, p<0.0001, p<0.0001$ and $p<0.00001$, respectively) and that Bulgarians do not statistically differ from Europeans and their European neighboring populations ( $p=0.896$ and $p=0.873002$, respectively).

\section{Discussion}

The modern Bulgarian gene pool has been gradually formed from a succession of populations with different genetic impacts. According to novel historical, linguistic and archeological data, one of the strongest genetic signals in the modern Bulgarian gene pool should derive from proto-Bulgarians (Dimitrov, 2005; Rashev, 1993).

It has been established that the modern Bulgarian mtDNA and Y-chromosome gene pools are overwhelmingly represented by Western Eurasian haplogroups. On the other hand, the Eastern Eurasian lineages among Bulgarians are represented by very low frequencies of mtDNA haplogroups $\mathrm{C}(0.2 \%), \mathrm{D}(0.4 \%)$ and $\mathrm{Z}(0.1 \%)$ and Y-chromosome haplogroups C, N and Q (each 0.5\%) (Karachanak et al., 2012, 2013). Similar to these findings, ancient mtDNA analysis of proto-Bulgarian human remains has revealed the presence of only Western Eurasian haplogroups (Nesheva et al., 2015). 
In order to further genetically test the hypotheses about the origin of modern-and proto-Bulgarians, in the present study we have analyzed the distribution of Y-chromosome haplogroups $\mathrm{C}, \mathrm{N}$ and Q and mtDNA haplogroups $\mathrm{C}, \mathrm{D}$ and $\mathrm{Z}$ across Eurasia and between modern Bulgarians and different population groups.

The frequency distributions of mtDNA haplogroups $\mathrm{C}, \mathrm{D}$ and $\mathrm{Z}$ and $\mathrm{Y}$-chromosome haplogroups $\mathrm{C}, \mathrm{N}$ and $\mathrm{Q}$ clearly depict their predominance in territories including those inhabited by Altaic (Turkic and Mongolic) populations. All of these haplogroups are underrepresented in Europe, with the exception of Y-chromosome haplogroup N, which is frequent only among East Slavic (Battaglia et al., 2009; Kushniarevich et al., 2013; Mirabal et al., 2009) populations and Swedish Saami (Karlsson et al., 2006) and is one of the features which genetically distinguish them from the remainder of the European populations.

The comparison of the incidence of $\mathrm{Y}$-chromosome haplogroups $\mathrm{C}, \mathrm{N}$ and $\mathrm{Q}$ points to a statistically significant difference between Bulgarians and Altaian, Siberian and Central Asian populations. Conversely, the chisquare test reveals a Y-chromosome link between Bulgarians and their European neighboring populations. The occurrence of mtDNA haplogroups $\mathrm{C}$ and D shows a similar pattern, with no association between Bulgarians and Altaian, Siberian, Central Asian and Caucasus populations and with similarity between Bulgarians and: their European neighboring populations and European populations as a whole. The discrepancy in the statistical significance of the distribution of Y-chromosome and mtDNA haplogroups in Bulgarians and Europeans is probably due to the mtDNA uniformity of Europe.

The Eurasian distribution of mtDNA haplogroups $\mathrm{C}, \mathrm{D}$ and $\mathrm{Z}$ and $\mathrm{Y}$-chromosome haplogroups $\mathrm{C}, \mathrm{N}$ and $\mathrm{Q}$ contrasts their high frequency among Altaic populations and their occasional appearance in Bulgarians. Furthermore, the comparison of the occurrence of these haplogroups shows no link between Altaic populations and Bulgarians. Based on the substantial genetic input of proto-Bulgarians to the modern Bulgarian gene pool, our findings confirm the nonexistence of a close Y-chromosomal or mtDNA link between proto-Bulgarians on one hand and Altaic and Caucasus populations on the other.

\section{Acknowledgements}

The study was supported by the National Science Fund of Bulgaria, project: "Characterization of the anthropo-genetic identity of Bulgarians", contract number DOO 2-110/22.05.2009.

\section{References}

Battaglia, V., Fornarino, S., Al-Zahery, N., Olivieri, A., Pala, M., Myres, N. M. et al. (2009). Y-Chromosomal Evidence of the Cultural Diffusion of Agriculture in Southeast Europe. European Journal of Human Genetics, 17, 820-830. http://dx.doi.org/10.1038/ejhg.2008.249

Calafell, F., Underhill, P., Tolun, A., Angelicheva, D., \& Kalaydjieva, L. (1996). From Asia to Europe: Mitochondrial DNA Sequence Variability in Bulgarians and Turks. Annals of Human Genetics, 60, 35-49. http://dx.doi.org/10.1111/j.1469-1809.1996.tb01170.x

Capelli, C., Brisighelli, F., Scarnicci, F., Arredi, B., Caglia, A., Vetrugno, G. et al. (2007). Y Chromosome Genetic Variation in the Italian Peninsula Is Clinal and Supports an Admixture Model for the Mesolithic-Neolithic Encounter. Molecular Phylogenetics and Evolution, 44, 228-239. http://dx.doi.org/10.1016/j.ympev.2006.11.030

Capelli, C., Redhead, N., Abernethy, J. K., Gratrix, F., Wilson, J. F., Moen, T. et al. (2003). A Y Chromosome Census of the British Isles. Current Biology, 13, 979-984. http://dx.doi.org/10.1016/S0960-9822(03)00373-7

Derenko, M., Malyarchuk, B., Denisova, G., Wozniak, M., Grzybowski, T., Dambueva, I., \& Zakharov, I. (2007a). Y-Chromosome Haplogroup N Dispersals from South Siberia to Europe. Journal of Human Genetics, 52, 763-770. http://dx.doi.org/10.1007/s10038-007-0179-5

Derenko, M., Malyarchuk, B., Grzybowski, T., Denisova, G., Dambueva, I., Perkova, M. et al. (2007b). Phylogeographic Analysis of Mitochondrial DNA in Northern Asian Populations. The American Journal of Human Genetics, 81, $1025-1041$. http://dx.doi.org/10.1086/522933

Derenko, M., Malyarchuk, B., Grzybowski, T., Denisova, G., Rogalla, U., Perkova, M. et al. (2010). Origin and Post-Glacial Dispersal of Mitochondrial DNA Haplogroups C and D in Northern Asia. PLoS ONE, 5, e15214.

http://dx.doi.org/10.1371/journal.pone.0015214

Dimitrov, B. (2005). 12 Myths in Bulgarian History. Sofia: KOM Foundation.

Dobrev, P. D. (1994). The World of the Proto-Bulgarians: Truths, Delusions. Sofia: Slavika-RM.

Dobrev, P. D. (2005). The Golden Core of the Bulgarian Antiquity. Sofia: Tangra TanNakRa IK. 
Francalacci, P., Morelli, L., Underhill, P. A., Lillie, A. S., Passarino, G., Useli, A. et al. (2003). Peopling of Three Mediterranean Islands (Corsica, Sardinia, and Sicily) Inferred by Y-Chromosome Biallelic Variability. American Journal of Physical Anthropology, 121, 270-279. http://dx.doi.org/10.1002/ajpa.10265

Gonçalves, R., Freitas, A., Branco, M., Rosa, A., Fernandes, A. T., Zhivotovsky, L. A. et al. (2005). Y-Chromosome Lineages from Portugal, Madeira and Acores Record Elements of Sephardim and Berber Ancestry. Annals of Human Genetics, 69, 443-454. http://dx.doi.org/10.1111/j.1529-8817.2005.00161.x

Grosheva, A., Shneider, Y. V., Zhukova, O., Morozova, I. Y., \& Rychkov, S. Y. (2014). Features of the Udmurt Mitochondrial Gene Pool in Relation to Tribal Structure. Russian Journal of Genetics, 50, 975-986. http://dx.doi.org/10.1134/S1022795414090063

Haefs, H. (2009). Das goldene Reich der Pamir-Bulgaren an Donau und Wardar. Nordtsedt: Books on Demand.

Hammer, M. F., Karafet, T. M., Park, H., Omoto, K., Harihara, S., Stoneking, M., \& Horai, S. (2005). Dual Origins of the Japanese: Common Ground for Hunter-Gatherer and Farmer Y Chromosomes. Journal of Human Genetics, 51, 47-58. http://dx.doi.org/10.1007/s10038-005-0322-0

Karachanak, S., Carossa, V., Nesheva, D., Olivieri, A., Pala, M., Hooshiar Kashani, B. et al. (2012). Bulgarians vs the Other European Populations: A Mitochondrial DNA Perspective. International Journal of Legal Medicine, 126, 497-503.

http://dx.doi.org/10.1007/s00414-011-0589-y

Karachanak, S., Grugni, V., Fornarino, S., Nesheva, D., Al-Zahery, N., Battaglia, V. et al. (2013). Y-Chromosome Diversity in Modern Bulgarians: New Clues about Their Ancestry. PLoS ONE, 8, e56779. http://dx.doi.org/10.1371/journal.pone.0056779

Karafet, T. M., Osipova, L. P., Gubina, M. A., Posukh, O. L., Zegura, S. L., \& Hammer, M. F. (2002). High Levels of YChromosome Differentiation among Native Siberian Populations and the Genetic Signature of a Boreal Hunter-Gatherer Way of Life. Human Biology, 74, 761-789. http://dx.doi.org/10.1353/hub.2003.0006

Karlsson, A. O., Wallerström, T., Götherström, A., \& Holmlund, G. (2006). Y-Chromosome Diversity in Sweden-A Long-Time Perspective. European Journal of Human Genetics, 14, 963-970. http://dx.doi.org/10.1038/sj.ejhg.5201651

King, R., Ozcan, S., Carter, T., Kalfoglu, E., Atasoy, S., Triantaphyllidis, C. et al. (2008). Differential Y-Chromosome Anatolian Influences on the Greek and Cretan Neolithic. Annals of Human Genetics, 72, 205-214. http://dx.doi.org/10.1111/j.1469-1809.2007.00414.x

Kushniarevich, A., Sivitskaya, L., Danilenko, N., Novogrodskii, T., Tsybovsky, I., Kiseleva, A. et al. (2013). Uniparental Genetic Heritage of Belarusians: Encounter of Rare Middle Eastern Matrilineages with a Central European Mitochondrial DNA Pool. PLoS ONE, 8, e66499. http://dx.doi.org/10.1371/journal.pone.0066499

Larmuseau, M. H., Vanderheyden, N., Jacobs, M., Coomans, M., Larno, L., \& Decorte, R. (2011). Micro-Geographic Distribution of Y-Chromosomal Variation in the Central-Western European Region Brabant. Forensic Science International: Genetics, 5, 95-99. http://dx.doi.org/10.1016/j.fsigen.2010.08.020

Liu, C., Wang, S.-Y., Zhao, M., Xu, Z.-Y., Hu, Y.-H., Chen, F. et al. (2011). Mitochondrial DNA Polymorphisms in Gelao Ethnic Group Residing in Southwest China. Forensic Science International: Genetics, 5, e4-e10. http://dx.doi.org/10.1016/j.fsigen.2010.04.007

López-Parra, A., Gusmao, L., Tavares, L., Baeza, C., Amorim, A., Mesa, M. et al. (2009). In Search of the Pre- and PostNeolithic Genetic Substrates in Iberia: Evidence from Y-Chromosome in Pyrenean Populations. Annals of Human Genetics, 73, 42-53. http://dx.doi.org/10.1111/j.1469-1809.2008.00478.x

Malyarchuk, B., Derenko, M., Denisova, G., Wozniak, M., Grzybowski, T., Dambueva, I., \& Zakharov, I. (2010). Phylogeography of the Y-Chromosome Haplogroup C in Northern Eurasia. Annals of Human Genetics, 74, 539-546. http://dx.doi.org/10.1111/j.1469-1809.2010.00601.x

Martinez, L., Underhill, P. A., Zhivotovsky, L. A., Gayden, T., Moschonas, N. K., Chow, C.-E. T. et al. (2007). Paleolithic Y-Haplogroup Heritage Predominates in a Cretan Highland Plateau. European Journal of Human Genetics, 15, 485-493. http://www.nature.com/ejhg/journal/v15/n4/suppinfo/5201769s1.html http://dx.doi.org/10.1038/sj.ejhg.5201769

Martinez-Cruz, B., Ioana, M., Calafell, F., Arauna, L. R., Sanz, P., Ionescu, R. et al. (2012). Y-Chromosome Analysis in Individuals Bearing the Basarab Name of the First Dynasty of Wallachian Kings. PLoS ONE, 7, e41803. http://dx.doi.org/10.1371/journal.pone.0041803

Mirabal, S., Regueiro, M., Cadenas, A. M., Cavalli-Sforza, L. L., Underhill, P. A., Verbenko, D. A. et al. (2009). Y-Chromosome Distribution within the Geo-Linguistic Landscape of Northwestern Russia. European Journal of Human Genetics, 17, 1260-1273. http://www.nature.com/ejhg/journal/v17/n10/suppinfo/ejhg20096s1.html http://dx.doi.org/10.1038/ejhg.2009.6 
Nesheva, D. V., Karachanak-Yankova, S., Lari, M., Yordanov, Y., Galabov, A., Caramelli, D., \& Toncheva, D. (2015). Mitochondrial DNA Suggests a Western Eurasian origin for Ancient (Proto-) Bulgarians. Human Biology Open Access Pre-Prints.

Nitecki, D. V., \& Nitecki, M. H. (2013). Origins of Anatomically Modern Humans. New York: Springer Science \& Business Media.

Noveski, P., Trivodalieva, S., Efremov, G., \& Plaseska-Karanfilska, D. (2010). Y Chromosome Single Nucleotide Polymorphisms Typing by SNaPshot Minisequencing. Balkan Journal of Medical Genetics, 13, 9-16. http://dx.doi.org/10.2478/v10034-010-0013-9

Quintana-Murci, L., Chaix, R., Wells, R. S., Behar, D. M., Sayar, H., Scozzari, R. et al. (2004). Where West Meets East: The Complex mtDNA Landscape of the Southwest and Central Asian Corridor. American Journal of Human Genetics, 74, 827-845. http://dx.doi.org/10.1086/383236

Rashev, R. (1993). On the Origin of the Proto-Bulgarians. In Studia protobulgarica et medievalia europensia: In Honor of Prof. V. Beshevliev. Veliko Turnovo: St. Cyril and St. Methodius University.

Ray, N., \& Adams, J. (2001). A GIS-Based Vegetation Map of the World at the Last Glacial Maximum (25,000-15,000 BP). Internet Archaeology, 11. http://dx.doi.org/10.11141/ia.11.2

Regueiro, M., Rivera, L., Damnjanovic, T., Lukovic, L., Milasin, J., \& Herrera, R. J. (2012). High Levels of Paleolithic Y-Chromosome Lineages Characterize Serbia. Gene, 498, 59-67. http://dx.doi.org/10.1016/j.gene.2012.01.030

Semino, O., Passarino, G., Oefner, P. J., Lin, A. A., Arbuzova, S., Beckman, L. E. et al. (2000). The Genetic Legacy of Paleolithic Homo Sapiens Sapiens in Extant Europeans: A Y Chromosome Perspective. Science, 290, 1155-1159. http://dx.doi.org/10.1126/science.290.5494.1155

Sengupta, S., Zhivotovsky, L. A., King, R., Mehdi, S., Edmonds, C. A., Chow, C.-E. T. et al. (2006). Polarity and Temporality of High-Resolution Y-Chromosome Distributions in India Identify Both Indigenous and Exogenous Expansions and Reveal Minor Genetic Influence of Central Asian Pastoralists. The American Journal of Human Genetics, 78, $202-221$. http://dx.doi.org/10.1086/499411

Sharma, S., Rai, E., Bhat, A. K., Bhanwer, A. S., \& Bamezai, R. N. (2007). A Novel Subgroup Q5 of Human Y-Chromosomal Haplogroup Q in India. BMC Evolutionary Biology, 7, 232. http://dx.doi.org/10.1186/1471-2148-7-232

Shi, H., Qi, X., Zhong, H., Peng, Y., Zhang, X., Ma, R. Z., \& Su, B. (2013). Genetic Evidence of an East Asian Origin and Paleolithic Northward Migration of Y-Chromosome Haplogroup N. PLoS ONE, 8, e66102. http://dx.doi.org/10.1371/journal.pone.0066102

Varzari, A., Kharkov, V., Nikitin, A. G., Raicu, F., Simonova, K., Stephan, W. et al. (2013). Paleo-Balkan and Slavic Contributions to the Genetic Pool of Moldavians: Insights from the Y Chromosome. PLoS ONE, 8, e53731. http://dx.doi.org/10.1371/journal.pone.0053731

Volodko, N. V., Starikovskaya, E. B., Mazunin, I. O., Eltsov, N. P., Naidenko, P. V., Wallace, D. C., \& Sukernik, R. I. (2008). Mitochondrial Genome Diversity in Arctic Siberians, with Particular Reference to the Evolutionary History of Beringia and Pleistocenic Peopling of the Americas. The American Journal of Human Genetics, 82, 1084-1100. http://dx.doi.org/10.1016/j.ajhg.2008.03.019

Yao, Y. G., Kong, Q. P., Wang, C. Y., Zhu, C. L., \& Zhang, Y. P. (2004). Different Matrilineal Contributions to Genetic Structure of Ethnic Groups in the Silk Road Region in China. Molecular Biology and Evolution, 21, 2265-2280. http://dx.doi.org/10.1093/molbev/msh238

Zhao, Y.-B., Sun, W.-Y., Zhan, Y., Wang, D., \& Yu, C.-C. (2011). Mitochondrial DNA Evidence of Southward Migration of Manchus in China. Molecular Biology, 45, 759-765. http://dx.doi.org/10.1093/molbev/msh238

Zhong, H., Shi, H., Qi, X.-B., Duan, Z.-Y., Tan, P.-P., Jin, L. et al. (2011). Extended Y Chromosome Investigation Suggests Postglacial Migrations of Modern Humans into East Asia via the Northern Route. Molecular Biology and Evolution, 28, 717-727. http://dx.doi.org/10.1093/molbev/msq247

Zlatarski, V. (1970). History of the Bulgarian State in the Middle Ages (Vol. 1). First Bulgarian Kingdom Part 1: Age of Hun-Bulgarian Supremacy. Sofia: Marin Drinov Academic Publishing House. 


\section{Supplementary}

Table S1. Absolute and percent frequencies of Y-chromosome haplogroups C (a), N (b) and Q (c) used to construct spatial frequency maps. N-number of analyzed individuals.

(a)

\begin{tabular}{|c|c|c|c|c|}
\hline Country & Reference & $\mathrm{N}$ & $\mathrm{Hg} \mathrm{C}$ & $\mathrm{Hg} \mathrm{C} \%$ \\
\hline Afghanistan-North & Lacau et al., 2012 & 44 & 0 & 0 \\
\hline Afghanistan-South & Lacau et al., 2012 & 146 & 5 & 3.42 \\
\hline Albania & Battaglia et al., 2009 & 55 & 0 & 0 \\
\hline Altai & Hammer et al., 2005 & 98 & 22 & 22.45 \\
\hline Altaian Kazakhs & Malyarchuk et al., 2010 & 36 & 21 & 58.33 \\
\hline Altaian Kazakhs (Southeastern) & Dulik et al., 2011 & 89 & 54 & 60.67 \\
\hline Altaian Kazakhs (Southwestern) & Dulik et al., 2011 & 30 & 17 & 56.67 \\
\hline Altaians & Malyarchuk et al., 2010 & 89 & 14 & 15.73 \\
\hline Anhui & Zhong et al., 2011 & 52 & 7 & 13.46 \\
\hline Antwerpen & Larmuseau et al., 2011 & 72 & 0 & 0 \\
\hline Balkarians & Battaglia et al., 2009 & 38 & 0 & 0 \\
\hline Belarussian-East Polesie & Kushniarevich et al., 2013 & 96 & 0 & 0 \\
\hline Belarussian-West Polesie & Kushniarevich et al., 2013 & 121 & 0 & 0 \\
\hline Belarussian-Centre & Kushniarevich et al., 2013 & 88 & 0 & 0 \\
\hline Belarussian-East & Kushniarevich et al., 2013 & 86 & 0 & 0 \\
\hline Belarussian-North & Kushniarevich et al., 2013 & 101 & 0 & 0 \\
\hline Belarussian-West & Kushniarevich et al., 2013 & 73 & 0 & 0 \\
\hline Bosniacs & Battaglia et al., 2009 & 84 & 0 & 0 \\
\hline Bosnian Croats & Battaglia et al., 2009 & 90 & 0 & 0 \\
\hline Bosnian Serbs & Battaglia et al., 2009 & 81 & 0 & 0 \\
\hline British Isles & Capelli et al., 2003 & 1772 & 0 & 0 \\
\hline Bulgaria & Karachanak et al., 2013 & 808 & 4 & 0.50 \\
\hline Buryat & Hammer et al., 2005 & 81 & 49 & 60.49 \\
\hline Buryats & Malyarchuk et al., 2010 & 217 & 148 & 68.20 \\
\hline Cambodia & Sengupta et al., 2006 & 6 & 0 & 0 \\
\hline China-Dai & Sengupta et al., 2006 & 7 & 2 & 28.57 \\
\hline China-Daur & Sengupta et al., 2006 & 7 & 2 & 28.57 \\
\hline China-Hezhen & Sengupta et al., 2006 & 6 & 3 & 50.00 \\
\hline China-Lahu & Sengupta et al., 2006 & 7 & 0 & 0 \\
\hline China-Naxi & Sengupta et al., 2006 & 8 & 0 & 0 \\
\hline China-Oroqen & Sengupta et al., 2006 & 7 & 5 & 71.43 \\
\hline
\end{tabular}




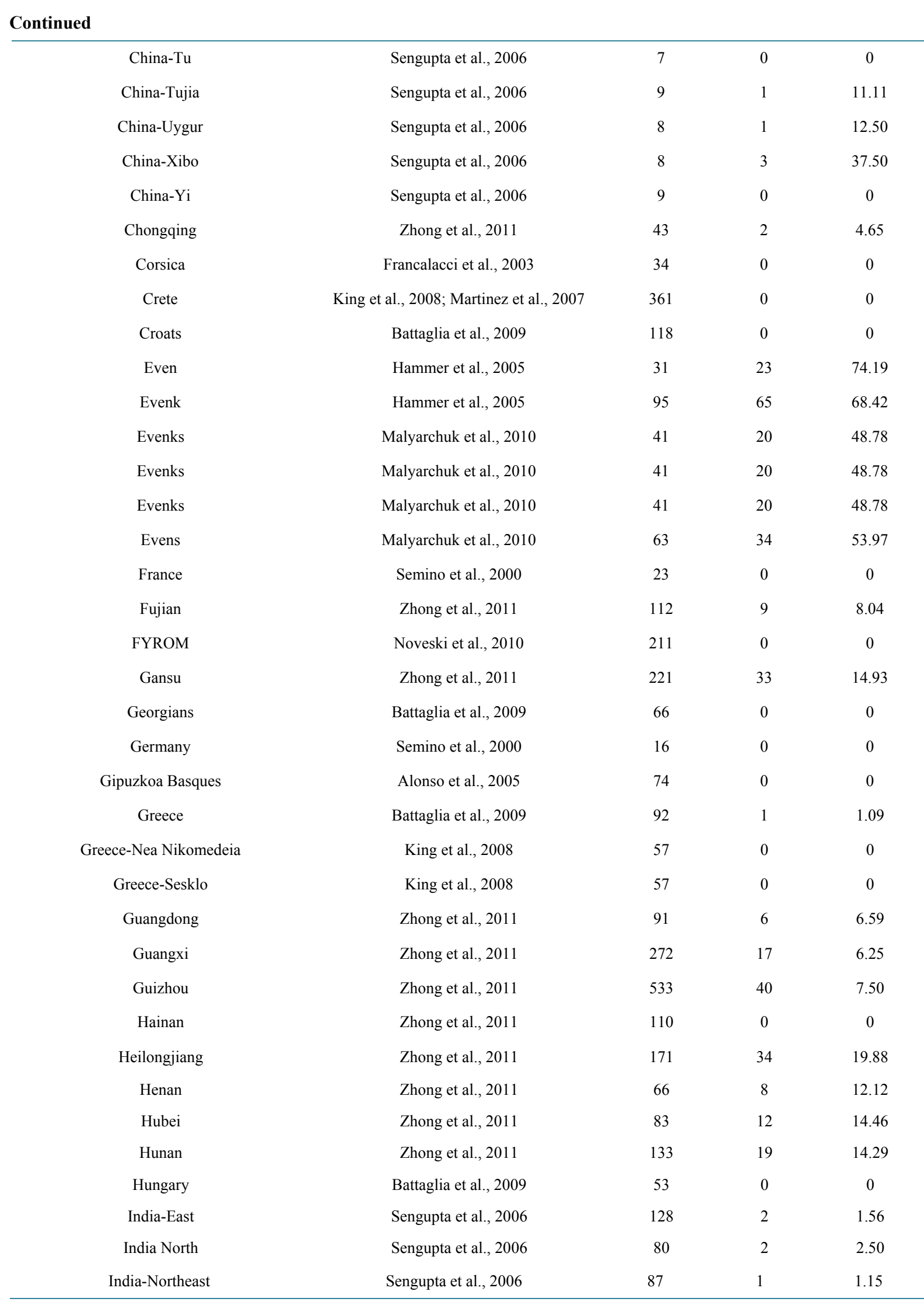




\section{Continued}

\begin{tabular}{|c|c|c|c|c|}
\hline India-Central & Sengupta et al., 2006 & 71 & 0 & 0 \\
\hline India-South & Sengupta et al., 2006 & 303 & 6 & 1.98 \\
\hline India-West & Sengupta et al., 2006 & 59 & 2 & 3.39 \\
\hline Indonesia-East & Hammer et al., 2005 & 55 & 18 & 32.73 \\
\hline Indonesia-West & Hammer et al., 2005 & 25 & 1 & 4.00 \\
\hline Inner Mongolia & Zhong et al., 2011 & 53 & 16 & 30.19 \\
\hline Iran & Grugni et al., 2012 & 827 & 9 & 1.09 \\
\hline Iran North & Regueiro et al., 2006 & 33 & 1 & 3.03 \\
\hline Iran South & Regueiro et al., 2006 & 117 & 0 & 0 \\
\hline Iraq (Marsh Arab) & Al-Zahery et al., 2011 & 143 & 0 & 0 \\
\hline Ireland & Moore et al., 2006 & 796 & 0 & 0 \\
\hline Italy-Apennine Marche & Capelli et al., 2007 & 27 & 0 & 0 \\
\hline Italy-Central Marche & Capelli et al., 2007 & 59 & 0 & 0 \\
\hline Italy-Central Tuscany & Capelli et al., 2007 & 41 & 0 & 0 \\
\hline Italy-Elba Island (Tuscany) & Capelli et al., 2007 & 95 & 0 & 0 \\
\hline Italy-North-East Latium & Capelli et al., 2007 & 55 & 0 & 0 \\
\hline Italy-North-West Apulia & Capelli et al., 2007 & 46 & 0 & 0 \\
\hline Italy-South Apulia & Capelli et al., 2007 & 71 & 0 & 0 \\
\hline Italy-South Latium & Capelli et al., 2007 & 51 & 0 & 0 \\
\hline Italy-Tuscany-Latium border & Capelli et al., 2007 & 79 & 0 & 0 \\
\hline Italy-Val Badia (Alto Adige) & Capelli et al., 2007 & 34 & 0 & 0 \\
\hline Italy-West Calabria & Capelli et al., 2007 & 57 & 0 & 0 \\
\hline Italy-West Campania & Capelli et al., 2007 & 84 & 0 & 0 \\
\hline Japan & Hammer et al., 2005 & 255 & 21 & 8.24 \\
\hline Jiangsu & Zhong et al., 2011 & 39 & 6 & 15.38 \\
\hline Jiangxi & Zhong et al., 2011 & 26 & 0 & 0 \\
\hline Jilin & Zhong et al., 2011 & 118 & 16 & 13.56 \\
\hline Jordan/Amman & Flores et al., 2005 & 101 & 0 & 0 \\
\hline Jordan/Dead Sea & Flores et al., 2005 & 45 & 0 & 0 \\
\hline Kalmyks & Malyarchuk et al., 2010 & 91 & 57 & 62.64 \\
\hline Kempen (Belgium) & Larmuseau et al., 2011 & 77 & 0 & 0 \\
\hline Khakassians & Malyarchuk et al., 2010 & 64 & 1 & 1.56 \\
\hline Khamnigans & Malyarchuk et al., 2010 & 51 & 28 & 54.90 \\
\hline Koreans & Malyarchuk et al., 2010 & 52 & 5 & 9.62 \\
\hline Koryaks & Malyarchuk et al., 2010 & 39 & 15 & 38.46 \\
\hline Liaoning & Zhong et al., 2011 & 149 & 34 & 22.82 \\
\hline Macedonian Greeks & Battaglia et al., 2009 & 57 & 0 & 0 \\
\hline
\end{tabular}




\begin{tabular}{|c|c|c|c|c|}
\hline \multicolumn{5}{|l|}{ Continued } \\
\hline Madeira & Gonc, alves et al., 2005 & 129 & 0 & 0 \\
\hline Malay & Hammer et al., 2005 & 32 & 1 & 3.13 \\
\hline Manchurian & Hammer et al., 2005 & 41 & 18 & 43.90 \\
\hline Mechelen (Belgium) & Larmuseau et al., 2011 & 63 & 0 & 0 \\
\hline Moldavia & Varzari et al., 2013 & 125 & 0 & 0 \\
\hline Mongolia-Outer & Hammer et al., 2005 & 149 & 78 & 52.35 \\
\hline Mongols & Malyarchuk et al., 2010 & 46 & 30 & 65.22 \\
\hline Ningxia & Zhong et al., 2011 & 62 & 11 & 17.74 \\
\hline Noord-Brabant (Netherlands) & Larmuseau et al., 2011 & 130 & 0 & 0 \\
\hline North Korea & Zhong et al., 2011 & 19 & 5 & 26.32 \\
\hline Norway & Capelli et al., 2003 & 201 & 0 & 0 \\
\hline Oman & Luis et al., 2004 & 121 & 4 & 3.31 \\
\hline Oroqen & Hammer et al., 2005 & 22 & 20 & 90.91 \\
\hline Pakistan-North & Sengupta et al., 2006 & 86 & 12 & 13.95 \\
\hline Pakistan-South & Sengupta et al., 2006 & 91 & 1 & 1.10 \\
\hline Philippines & Hammer et al., 2005 & 48 & 1 & 2.08 \\
\hline Poland & Battaglia et al., 2009 & 99 & 0 & 0 \\
\hline Portugal-Central & Gonc,alves et al., 2005 & 102 & 0 & 0 \\
\hline Portugal-North & Gonc, alves et al., 2005 & 101 & 1 & 0.99 \\
\hline Portugal-South & Gonc, alves et al., 2005 & 100 & 1 & 1.00 \\
\hline Qinghai & Zhong et al., 2011 & 40 & 3 & 7.50 \\
\hline Romania & Martinez-Cruz et al., 2012 & 149 & 0 & 0 \\
\hline Russia-Arkhangelski & Mirabal et al., 2009 & 28 & 0 & 0 \\
\hline Russia-Belgorod & Balanovsky et al., 2008 & 143 & 1 & 0.70 \\
\hline Russia-Izhemski Komi & Mirabal et al., 2009 & 54 & 0 & 0 \\
\hline Russia-Kashin & Balanovsky et al., 2008 & 73 & 0 & 0 \\
\hline Russia-Krasnoborsk & Balanovsky et al., 2008 & 91 & 0 & 0 \\
\hline Russia-Kuban Cossacs & Balanovsky et al., 2008 & 90 & 0 & 0 \\
\hline Russia-Kursk & Mirabal et al., 2009 & 40 & 0 & 0 \\
\hline Russia-Livni & Balanovsky et al., 2008 & 110 & 0 & 0 \\
\hline Russia-Mezen & Balanovsky et al., 2008 & 54 & 0 & 0 \\
\hline Russians & Malyarchuk et al., 2010 & 406 & 3 & 0.74 \\
\hline Russia-Ostrov & Balanovsky et al., 2008 & 75 & 0 & 0 \\
\hline Russia-Pinega & Balanovsky et al., 2008 & 114 & 0 & 0 \\
\hline Russia-Porhov & Balanovsky et al., 2008 & 57 & 0 & 0 \\
\hline Russia-Priluzski Komi & Mirabal et al., 2009 & 49 & 0 & 0 \\
\hline Russia-Pristen & Balanovsky et al., 2008 & 45 & 0 & 0 \\
\hline
\end{tabular}




\section{Continued}

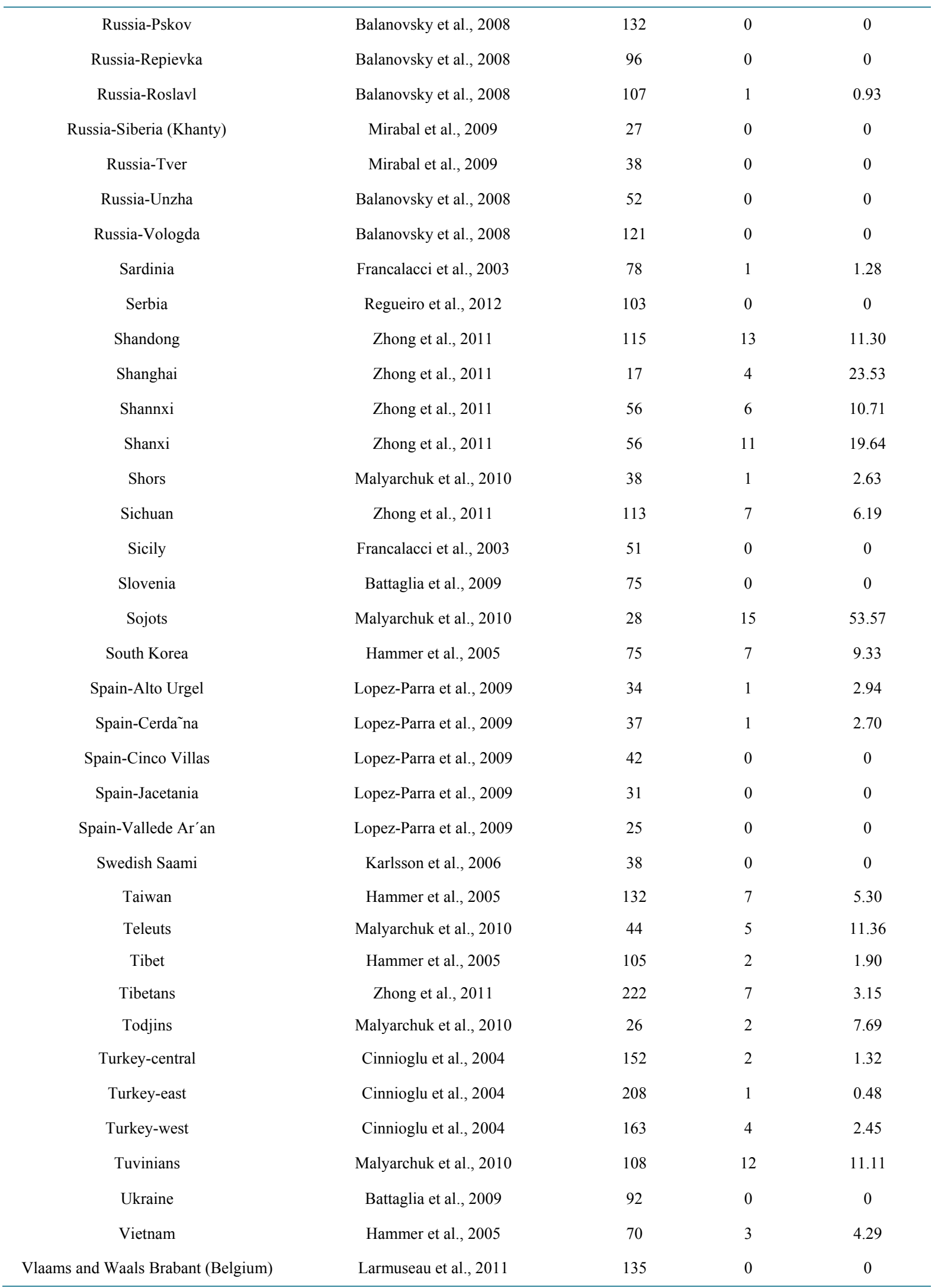




\section{Continued}

\begin{tabular}{|c|c|c|c|c|}
\hline Xingjiang & Zhong et al., 2011 & 244 & 54 & 22.13 \\
\hline Xinjiang & Zhong et al., 2011 & 61 & 22 & 36.07 \\
\hline Yakut & Sengupta et al., 2006 & 18 & 2 & 11.11 \\
\hline Yakuts & Malyarchuk et al., 2010 & 10 & 2 & 20.00 \\
\hline Yemen & Cadenas et al., 2008 & 62 & 0 & 0 \\
\hline Yunnan & Zhong et al., 2011 & 447 & 41 & 9.17 \\
\hline Zhejiang & Zhong et al., 2011 & 102 & 5 & 4.90 \\
\hline
\end{tabular}

\section{References S1(a)}

Alonso, S., Flores, C., Cabrera, V., Alonso, A., Martin, P., Albarran, C. et al. (2005). The Place of the Basques in the European Y-Chromosome Diversity Landscape. European Journal of Human Genetics, 13, 1293-1302.

http://dx.doi.org/10.1038/sj.ejhg.5201482

Al-Zahery, N., Pala, M., Battaglia, V., Grugni, V., Hamod, M. A., Kashani, B. H. et al. (2011). In Search of the Genetic Footprints of Sumerians: A Survey of Y-Chromosome and mtDNA Variation in the Marsh Arabs of Iraq. BMC Evolutionary Biology, 11, 288. http://dx.doi.org/10.1186/1471-2148-11-288

Balanovsky, O., Rootsi, S., Pshenichnov, A., Kivisild, T., Churnosov, M., Evseeva, I. et al. (2008). Two Sources of the Russian Patrilineal Heritage in Their Eurasian Context. American Journal of Human Genetics, 82, 236-250. http://dx.doi.org/10.1016/j.ajhg.2007.09.019

Battaglia, V., Fornarino, S., Al-Zahery, N., Olivieri, A., Pala, M., Myres, N. M. et al. (2009). Y-Chromosomal Evidence of the Cultural Diffusion of Agriculture in Southeast Europe. European Journal of Human Genetics, 17, 820-830. http://dx.doi.org/10.1038/ejhg.2008.249

Cadenas, A. M., Zhivotovsky, L. A., Cavalli-Sforza, L. L., Underhill, P. A., \& Herrera, R. J. (2008). Y-Chromosome Diversity Characterizes the Gulf of Oman. European Journal of Human Genetics, 16, 374-386. http://dx.doi.org/10.1038/sj.ejhg.5201934

Capelli, C., Brisighelli, F., Scarnicci, F., Arredi, B., Caglia, A., Vetrugno, G. et al. (2007). Y Chromosome Genetic Variation in the Italian Peninsula Is Clinal and Supports an Admixture Model for the Mesolithic-Neolithic Encounter. Molecular Phylogenetics and Evolution, 44, 228-239. http://dx.doi.org/10.1016/j.ympev.2006.11.030

Capelli, C., Redhead, N., Abernethy, J. K., Gratrix, F., Wilson, J. F., Moen, T. et al. (2003). A Y Chromosome Census of the British Isles. Current Biology, 13, 979-984. http://dx.doi.org/10.1016/s0960-9822(03)00373-7

Cinnioğlu, C., King, R., Kivisild, T., Kalfoğlu, E., Atasoy, S., Cavalleri, G. L. et al. (2004). Excavating Y-Chromosome Haplotype Strata in Anatolia. Human Genetics, 114, 127-148. http://dx.doi.org/10.1007/s00439-003-1031-4

Dulik, M. C., Osipova, L. P., \& Schurr, T. G. (2011). Y-Chromosome Variation in Altaian Kazakhs Reveals a Common Paternal Gene Pool for Kazakhs and the Influence of Mongolian Expansions. PLoS ONE, 6, e17548. http://dx.doi.org/10.1371/journal.pone.0017548

Flores, C., Maca-Meyer, N., Larruga, J. M., Cabrera, V. M., Karadsheh, N., \& Gonzalez, A. M. (2005). Isolates in a Corridor of Migrations: A High-Resolution Analysis of Y-Chromosome Variation in Jordan. Journal of Human Genetics, 50, 435441. http://dx.doi.org/10.1007/s10038-005-0274-4

Francalacci, P., Morelli, L., Underhill, P. A., Lillie, A. S., Passarino, G., Useli, A. et al. (2003). Peopling of Three Mediterranean Islands (Corsica, Sardinia, and Sicily) Inferred by Y-Chromosome Biallelic Variability. American Journal of Physical Anthropology, 121, 270-279. http://dx.doi.org/10.1002/ajpa.10265

Gonçalves, R., Freitas, A., Branco, M., Rosa, A., Fernandes, A. T., Zhivotovsky, L. A. et al. (2005). Y-Chromosome Lineages from Portugal, Madeira and Açores Record Elements of Sephardim and Berber Ancestry. Annals of Human Genetics, 69, 443-454. http://dx.doi.org/10.1111/j.1529-8817.2005.00161.x

Grugni, V., Battaglia, V., Hooshiar Kashani, B., Parolo, S., Al-Zahery, N., Achilli, A. et al. (2012). Ancient Migratory Events in the Middle East: New Clues from the Y-Chromosome Variation of Modern Iranians. PLoS ONE, 7, e41252. http://dx.doi.org/10.1371/journal.pone.0041252

Hammer, M. F., Karafet, T. M., Park, H., Omoto, K., Harihara, S., Stoneking, M., \& Horai, S. (2005). Dual Origins of the Japanese: Common Ground for Hunter-Gatherer and Farmer Y Chromosomes. Journal of Human Genetics, 51, 47-58. http://dx.doi.org/10.1007/s10038-005-0322-0

Karachanak, S., Grugni, V., Fornarino, S., Nesheva, D., Al-Zahery, N., Battaglia, V. et al. (2013). Y-Chromosome Diversity in Modern Bulgarians: New Clues about Their Ancestry. PLoS ONE, 8, e56779. 
http://dx.doi.org/10.1371/journal.pone.0056779

Karlsson, A. O., Wallerström, T., Götherström, A., \& Holmlund, G. (2006). Y-Chromosome Diversity in Sweden-A LongTime Perspective. European Journal of Human Genetics, 14, 963-970. http://dx.doi.org/10.1038/sj.ejhg.5201651

King, R., Özcan, S., Carter, T., Kalfoğlu, E., Atasoy, S., Triantaphyllidis, C. et al. (2008). Differential Y-Chromosome Anatolian Influences on the Greek and Cretan Neolithic. Annals of Human Genetics, 72, 205-214. http://dx.doi.org/10.1111/j.1469-1809.2007.00414.x

Kushniarevich, A., Sivitskaya, L., Danilenko, N., Novogrodskii, T., Tsybovsky, I., Kiseleva, A. et al. (2013). Uniparental Genetic Heritage of Belarusians: Encounter of Rare Middle Eastern Matrilineages with a Central European Mitochondrial DNA Pool. PLoS ONE, 8, e66499. http://dx.doi.org/10.1371/journal.pone.0066499

Lacau, H., Gayden, T., Regueiro, M., Chennakrishnaiah, S., Bukhari, A., Underhill, P. A. et al. (2012). Afghanistan from a Y-Chromosome Perspective. European Journal of Human Genetics, 20, 1063-1070. http://dx.doi.org/10.1038/ejhg.2012.59

Larmuseau, M. H., Vanderheyden, N., Jacobs, M., Coomans, M., Larno, L., \& Decorte, R. (2011). Micro-Geographic Distribution of Y-Chromosomal Variation in the Central-Western European Region Brabant. Forensic Science International: Genetics, 5, 95-99. http://dx.doi.org/10.1016/j.fsigen.2010.08.020

López-Parra, A., Gusmao, L., Tavares, L., Baeza, C., Amorim, A., Mesa, M. et al. (2009). In Search of the Pre- and Post-Neolithic Genetic Substrates in Iberia: Evidence from Y-Chromosome in Pyrenean Populations. Annals of Human Genetics, 73, 42-53. http://dx.doi.org/10.1111/j.1469-1809.2008.00478.x

Luis, J. R., Rowold, D. J., Regueiro, M., Caeiro, B., Cinnioğlu, C., Roseman, C. et al. (2004). The Levant versus the Horn of Africa: Evidence for Bidirectional Corridors of Human Migrations. The American Journal of Human Genetics, 74, 532544. http://dx.doi.org/10.1086/382286

Malyarchuk, B., Derenko, M., Denisova, G., Wozniak, M., Grzybowski, T., Dambueva, I., \& Zakharov, I. (2010). Phylogeography of the Y-Chromosome Haplogroup C in Northern Eurasia. Annals of Human Genetics, 74, 539-546. http://dx.doi.org/10.1111/j.1469-1809.2010.00601.x

Martinez, L., Underhill, P. A., Zhivotovsky, L. A., Gayden, T., Moschonas, N. K., Chow, C.-E. T. et al. (2007). Paleolithic Y-Haplogroup Heritage Predominates in a Cretan Highland Plateau. European Journal of Human Genetics, 15, 485-493. http://www.nature.com/ejhg/journal/v15/n4/suppinfo/5201769s1.html http://dx.doi.org/10.1038/sj.ejhg.5201769

Martinez-Cruz, B., Ioana, M., Calafell, F., Arauna, L. R., Sanz, P., Ionescu, R. et al. (2012). Y-Chromosome Analysis in Individuals Bearing the Basarab Name of the First Dynasty of Wallachian Kings. PLoS ONE, 7, e41803. http://dx.doi.org/10.1371/journal.pone.0041803

Mirabal, S., Regueiro, M., Cadenas, A. M., Cavalli-Sforza, L. L., Underhill, P. A., Verbenko, D. A. et al. (2009). YChromosome Distribution within the Geo-Linguistic Landscape of Northwestern Russia. European Journal of Human Genetics, 17, 1260-1273. http://www.nature.com/ejhg/journal/v17/n10/suppinfo/ejhg20096s1.html http://dx.doi.org/10.1038/ejhg.2009.6

Noveski, P., Trivodalieva, S., Efremov, G., \& Plaseska-Karanfilska, D. (2010). Y Chromosome Single Nucleotide Polymorphisms Typing by SNaPshot Minisequencing. Balkan Journal of Medical Genetics, 13, 9-16. http://dx.doi.org/10.2478/v10034-010-0013-9

Regueiro, M., Cadenas, A., Gayden, T., Underhill, P., \& Herrera, R. (2006). Iran: Tricontinental Nexus for Y-Chromosome Driven Migration. Human Heredity, 61, 132-143. http://dx.doi.org/10.1159/000093774

Regueiro, M., Rivera, L., Damnjanovic, T., Lukovic, L., Milasin, J., \& Herrera, R. J. (2012). High Levels of Paleolithic Y-Chromosome Lineages Characterize Serbia. Gene, 498, 59-67. http://dx.doi.org/10.1016/j.gene.2012.01.030

Semino, O., Passarino, G., Oefner, P. J., Lin, A. A., Arbuzova, S., Beckman, L. E. et al. (2000). The Genetic Legacy of Paleolithic Homo Sapiens Sapiens in Extant Europeans: A Y Chromosome Perspective. Science, 290, 1155-1159. http://dx.doi.org/10.1126/science.290.5494.1155

Sengupta, S., Zhivotovsky, L. A., King, R., Mehdi, S., Edmonds, C. A., Chow, C.-E. T. et al. (2006). Polarity and Temporality of High-Resolution Y-Chromosome Distributions in India Identify Both Indigenous and Exogenous Expansions and Reveal Minor Genetic Influence of Central Asian Pastoralists. The American Journal of Human Genetics, 78, 202-221. http://dx.doi.org/10.1086/499411

Varzari, A., Kharkov, V., Nikitin, A. G., Raicu, F., Simonova, K., Stephan, W. et al. (2013). Paleo-Balkan and Slavic Contributions to the Genetic Pool of Moldavians: Insights from the Y Chromosome. PLoS ONE, 8, e53731. http://dx.doi.org/10.1371/journal.pone.0053731

Zhong, H., Shi, H., Qi, X.-B., Duan, Z.-Y., Tan, P.-P., Jin, L. et al. (2011). Extended Y Chromosome Investigation Suggests Postglacial Migrations of Modern Humans into East Asia via the Northern Route. Molecular Biology and Evolution, 28, 717-727. http://dx.doi.org/10.1093/molbev/msq247 
(b)

\begin{tabular}{|c|c|c|c|c|}
\hline Country & Reference & $\mathrm{N}$ & $\mathrm{Hg} \mathrm{N}$ & $\mathrm{Hg} \mathrm{N} \%$ \\
\hline Afghanistan-North & Lacau et al., 2012 & 44 & 0 & 0 \\
\hline Afghanistan-South & Lacau et al., 2012 & 146 & 0 & 0 \\
\hline Albania & Battaglia et al., 2009 & 55 & 0 & 0 \\
\hline Altai & Hammer et al., 2005 & 98 & 4 & 4.08 \\
\hline Altaian Kazakhs (Southeastern) & Dulik et al., 2011 & 89 & 0 & 0 \\
\hline Altaian Kazakhs (Southwestern) & Dulik et al., 2011 & 30 & 0 & 0 \\
\hline Altaians & Derenko et al., 2007 & 142 & 13 & 9.15 \\
\hline Anhui & Zhong et al., 2011 & 52 & 4 & 7.69 \\
\hline Antwerpen & Larmuseau et al., 2011 & 72 & 0 & 0 \\
\hline Balkarians & Battaglia et al., 2009 & 38 & 0 & 0 \\
\hline Belarussian-East Polesie & Kushniarevich et al., 2013 & 96 & 8 & 8.33 \\
\hline Belarussian-West Polesie & Kushniarevich et al., 2013 & 121 & 10 & 8.26 \\
\hline Belarussian-Centre & Kushniarevich et al., 2013 & 88 & 7 & 7.95 \\
\hline Belarussian-East & Kushniarevich et al., 2013 & 86 & 6 & 6.98 \\
\hline Belarussian-North & Kushniarevich et al., 2013 & 101 & 16 & 15.84 \\
\hline Belarussian-West & Kushniarevich et al., 2013 & 73 & 10 & 13.70 \\
\hline Bosniacs & Battaglia et al., 2009 & 84 & 0 & 0 \\
\hline Bosnian Croats & Battaglia et al., 2009 & 90 & 0 & 0 \\
\hline Bosnian Serbs & Battaglia et al., 2009 & 81 & 5 & 6.17 \\
\hline Bulgaria & Karachanak et al., 2013 & 808 & 4 & 0.50 \\
\hline Buryat & Hammer et al., 2005 & 81 & 25 & 30.86 \\
\hline Buryats & Derenko et al., 2007 & 217 & 41 & 18.89 \\
\hline Cambodia & Sengupta et al., 2006 & 6 & 1 & 16.67 \\
\hline China-Dai & Sengupta et al., 2006 & 7 & 0 & 0 \\
\hline China-Daur & Sengupta et al., 2006 & 7 & 1 & 14.29 \\
\hline China-Hezhen & Sengupta et al., 2006 & 6 & 0 & 0 \\
\hline China-Lahu & Sengupta et al., 2006 & 7 & 0 & 0 \\
\hline China-Naxi & Sengupta et al., 2006 & 8 & 2 & 25.00 \\
\hline China-Oroqen & Sengupta et al., 2006 & 7 & 2 & 28.57 \\
\hline China-Tu & Sengupta et al., 2006 & 7 & 2 & 28.57 \\
\hline China-Tujia & Sengupta et al., 2006 & 9 & 1 & 11.11 \\
\hline China-Uygur & Sengupta et al., 2006 & 8 & 0 & 0 \\
\hline China-Xibo & Sengupta et al., 2006 & 8 & 1 & 12.50 \\
\hline China-Yi & Sengupta et al., 2006 & 9 & 1 & 11.11 \\
\hline Chongqing & Zhong et al., 2011 & 43 & 1 & 2.33 \\
\hline Corsica & Francalacci et al., 2003 & 34 & 0 & 0 \\
\hline Crete & Martinez et al., 2007; King et al., 2008 & 361 & 0 & 0 \\
\hline Croats & Battaglia et al., 2009 & 118 & 2 & 1.69 \\
\hline
\end{tabular}




\section{Continued}

\begin{tabular}{|c|c|c|c|c|}
\hline Czechs & Battaglia et al., 2009 & 75 & 2 & 2.67 \\
\hline Even & Hammer et al., 2005 & 31 & 4 & 12.90 \\
\hline Evenk & Hammer et al., 2005 & 95 & 18 & 18.95 \\
\hline Evenks & Derenko et al., 2007 & 41 & 12 & 29.27 \\
\hline Evenks & Derenko et al., 2007 & 41 & 12 & 29.27 \\
\hline Evenks & Derenko et al., 2007 & 41 & 12 & 29.27 \\
\hline Evens & Derenko et al., 2007 & 11 & 1 & 9.09 \\
\hline France & Semino et al., 2000 & 23 & 0 & 0 \\
\hline Fujian & Zhong et al., 2011 & 112 & 6 & 5.36 \\
\hline FYROM & Noveski et al., 2010 & 211 & 1 & 0.47 \\
\hline Gansu & Zhong et al., 2011 & 221 & 15 & 6.79 \\
\hline Georgians & Battaglia et al., 2009 & 66 & 0 & 0 \\
\hline Germany & Semino et al., 2000 & 16 & 0 & 0 \\
\hline Gipuzkoa Basques & Alonso et al., 2004 & 74 & 0 & 0 \\
\hline Greece & Battaglia et al., 2009 & 92 & 0 & 0 \\
\hline Greece-Nea Nikomedeia & King et al., 2008 & 57 & 0 & 0 \\
\hline Greece-Sesklo & King et al., 2008 & 57 & 0 & 0 \\
\hline Guangdong & Zhong et al., 2011 & 91 & 4 & 4.40 \\
\hline Guangxi & Zhong et al., 2011 & 272 & 6 & 2.21 \\
\hline Guizhou & Zhong et al., 2011 & 533 & 27 & 5.07 \\
\hline Hainan & Zhong et al., 2011 & 110 & 2 & 1.82 \\
\hline Heilongjiang & Zhong et al., 2011 & 171 & 10 & 5.85 \\
\hline Henan & Zhong et al., 2011 & 66 & 3 & 4.55 \\
\hline Hubei & Zhong et al., 2011 & 83 & 7 & 8.43 \\
\hline Hunan & Zhong et al., 2011 & 133 & 12 & 9.02 \\
\hline Hungary & Battaglia et al., 2009 & 53 & 0 & 0 \\
\hline India-East & Sengupta et al., 2006 & 128 & 0 & 0 \\
\hline India North & Sengupta et al., 2006 & 80 & 0 & 0 \\
\hline India-Northeast & Sengupta et al., 2006 & 87 & 0 & 0 \\
\hline India-Central & Sengupta et al., 2006 & 71 & 0 & 0 \\
\hline India-South & Sengupta et al., 2006 & 303 & 0 & 0 \\
\hline India-West & Sengupta et al., 2006 & 59 & 0 & 0 \\
\hline Indonesia-East & Hammer et al., 2005 & 55 & 0 & 0 \\
\hline Indonesia-West & Hammer et al., 2005 & 25 & 0 & 0 \\
\hline Inner Mongolia & Zhong et al., 2011 & 53 & 5 & 9.43 \\
\hline Iran & Grugni et al., 2012 & 827 & 3 & 0.36 \\
\hline Iran North & Regueiro et al., 2006 & 33 & 2 & 6.06 \\
\hline
\end{tabular}




\section{Continued}

\begin{tabular}{|c|c|c|c|c|}
\hline Iran South & Regueiro et al., 2006 & 117 & 1 & 0.85 \\
\hline Iraq (Marsh Arab) & Al-Zahery et al., 2011 & 143 & 0 & 0 \\
\hline Italy-Central Marche & Capelli et al., 2007 & 59 & 0 & 0 \\
\hline Italy-Elba Island (Tuscany) & Capelli et al., 2007 & 95 & 0 & 0 \\
\hline Italy-West Calabria & Capelli et al., 2007 & 57 & 0 & 0 \\
\hline Jiangsu & Zhong et al., 2011 & 39 & 0 & 0 \\
\hline Jiangxi & Zhong et al., 2011 & 26 & 0 & 0 \\
\hline Jilin & Zhong et al., 2011 & 118 & 9 & 7.63 \\
\hline Jordan/Amman & Flores et al., 2005 & 101 & 0 & 0 \\
\hline Kempen (Belgium) & Larmuseau et al., 2011 & 77 & 1 & 1.30 \\
\hline Khakassians & Derenko et al., 2007 & 64 & 32 & 50.00 \\
\hline Koreans & Derenko et al., 2007 & 52 & 0 & 0 \\
\hline Koryaks & Derenko et al., 2007 & 16 & 4 & 25.00 \\
\hline Liaoning & Zhong et al., 2011 & 149 & 13 & 8.72 \\
\hline Macedonian Greeks & Battaglia et al., 2009 & 57 & 0 & 0 \\
\hline Madeira & Gonc, alves et al., 2005 & 129 & 3 & 2.33 \\
\hline Mongolia-Outer & Hammer et al., 2005 & 149 & 13 & 8.72 \\
\hline Ningxia & Zhong et al., 2011 & 62 & 5 & 8.06 \\
\hline Noord-Brabant (Netherlands) & Larmuseau et al., 2011 & 130 & 0 & 0 \\
\hline North Korea & Zhong et al., 2011 & 19 & 1 & 5.26 \\
\hline Norway & Capelli et al., 2003 & 201 & 2 & 1.00 \\
\hline Oman & Luis et al., 2004 & 121 & 0 & 0 \\
\hline Oroqen & Hammer et al., 2005 & 22 & 1 & 4.55 \\
\hline Pakistan-North & Sengupta et al., 2006 & 86 & 0 & 0 \\
\hline Pakistan-South & Sengupta et al., 2006 & 91 & 0 & 0 \\
\hline Philippines & Hammer et al., 2005 & 48 & 0 & 0 \\
\hline Poland & Battaglia et al., 2009 & 99 & 1 & 1.01 \\
\hline Portugal-Central & Gonc,alves et al., 2005 & 102 & 0 & 0 \\
\hline Portugal-North & Gonc,alves et al., 2005 & 101 & 0 & 0 \\
\hline Portugal-South & Gonc, alves et al., 2005 & 100 & 0 & 0 \\
\hline
\end{tabular}




\section{Continued}

\begin{tabular}{|c|c|c|c|c|}
\hline Russia-Kashin & Balanovsky et al., 2008 & 73 & 8 & 10.96 \\
\hline Russia-Krasnoborsk & Balanovsky et al., 2008 & 91 & 36 & 39.56 \\
\hline Russia-Kuban Cossacs & Balanovsky et al., 2008 & 90 & 7 & 7.78 \\
\hline Russia-Kursk & Mirabal et al., 2009 & 40 & 37 & 92.50 \\
\hline Russia-Livni & Balanovsky et al., 2008 & 110 & 6 & 5.45 \\
\hline Russia-Mezen & Balanovsky et al., 2008 & 54 & 29 & 53.70 \\
\hline Russia-Ostrov & Balanovsky et al., 2008 & 75 & 21 & 28.00 \\
\hline Russia-Pinega & Balanovsky et al., 2008 & 114 & 45 & 39.47 \\
\hline Russia-Porhov & Balanovsky et al., 2008 & 57 & 11 & 19.30 \\
\hline Russia-Priluzski Komi & Mirabal et al., 2009 & 49 & 5 & 10.20 \\
\hline Russia-Pristen & Balanovsky et al., 2008 & 45 & 6 & 13.33 \\
\hline Russia-Pskov & Balanovsky et al., 2008 & 132 & 32 & 24.24 \\
\hline Russia-Repievka & Balanovsky et al., 2008 & 96 & 6 & 6.25 \\
\hline Russia-Roslavl & Balanovsky et al., 2008 & 107 & 15 & 14.02 \\
\hline Russia-Siberia (Khanty) & Mirabal et al., 2009 & 27 & 22 & 81.48 \\
\hline Russia-Tver & Mirabal et al., 2009 & 38 & 30 & 78.95 \\
\hline Russia-Unzha & Balanovsky et al., 2008 & 52 & 7 & 13.46 \\
\hline Russia-Vologda & Balanovsky et al., 2008 & 121 & 47 & 38.84 \\
\hline Sardinia & Francalacci et al., 2003 & 78 & 0 & 0 \\
\hline Serbia & Regueiro et al., 2012 & 103 & 2 & 1.94 \\
\hline Shandong & Zhong et al., 2011 & 115 & 10 & 8.70 \\
\hline Shanghai & Zhong et al., 2011 & 17 & 0 & 0 \\
\hline Shannxi & Zhong et al., 2011 & 56 & 0 & 0 \\
\hline Shanxi & Zhong et al., 2011 & 56 & 12 & 21.43 \\
\hline Shors & Derenko et al., 2007 & 38 & 7 & 18.42 \\
\hline Sichuan & Zhong et al., 2011 & 113 & 15 & 13.27 \\
\hline Sicily & Francalacci et al., 2003 & 51 & 0 & 0 \\
\hline Slovenia & Battaglia et al., 2009 & 75 & 0 & 0 \\
\hline Sojots & Derenko et al., 2007 & 28 & 3 & 10.71 \\
\hline South Korea & Hammer et al., 2005 & 75 & 2 & 2.67 \\
\hline Spain-Alto Urgel & Lopez-Parra et al., 2009 & 34 & 0 & 0 \\
\hline Spain-Cerda na & Lopez-Parra et al., 2009 & 37 & 0 & 0 \\
\hline Spain-Cinco Villas & Lopez-Parra et al., 2009 & 42 & 0 & 0 \\
\hline Spain-Jacetania & Lopez-Parra et al., 2009 & 31 & 0 & 0 \\
\hline Spain-Vallede Ar'an & Lopez-Parra et al., 2009 & 25 & 0 & 0 \\
\hline Swedish Saami & Karlsson et al., 2006 & 38 & 17 & 44.74 \\
\hline Taiwan & Hammer et al., 2005 & 132 & 5 & 3.79 \\
\hline Tajiks & Derenko et al., 2007 & 103 & 0 & 0 \\
\hline Tibet & Hammer et al., 2005 & 105 & 3 & 2.86 \\
\hline Tibetans & Zhong et al., 2011 & 222 & 4 & 1.80 \\
\hline Tofalars & Derenko et al., 2007 & 30 & 21 & 70.00 \\
\hline Turkey-central & Cinnioglu et al., 2004 & 152 & 7 & 4.61 \\
\hline Turkey-east & Cinnioglu et al., 2004 & 208 & 8 & 3.85 \\
\hline
\end{tabular}




\begin{tabular}{|c|c|c|c|c|}
\hline \multicolumn{5}{|l|}{ Continued } \\
\hline Turkey-west & Cinnioglu et al., 2004 & 163 & 5 & 3.07 \\
\hline Tuvinians & Derenko et al., 2007 & 134 & 39 & 29.10 \\
\hline Ukraine & Battaglia et al., 2009 & 92 & 6 & 6.52 \\
\hline Vietnam & Hammer et al., 2005 & 70 & 2 & 2.86 \\
\hline Vlaams and Waals Brabant (Belgium) & Larmuseau et al., 2011 & 135 & 1 & 0.74 \\
\hline Xingjiang & Zhong et al., 2011 & 244 & 9 & 3.69 \\
\hline Xinjiang & Zhong et al., 2011 & 61 & 11 & 18.03 \\
\hline Yakuts & Derenko et al., 2007; Sengupta et al., 2006 & 28 & 24 & 84.21 \\
\hline Yemen & Cadenas et al., 2008 & 62 & 0 & 0 \\
\hline Yunnan & Zhong et al., 2011 & 447 & 48 & 10.74 \\
\hline Zhejiang & Zhong et al., 2011 & 102 & 9 & 8.82 \\
\hline
\end{tabular}

\section{References S1(b)}

Alonso, S., Flores, C., Cabrera, V., Alonso, A., Martin, P., Albarran, C. et al. (2005). The Place of the Basques in the European Y-Chromosome Diversity Landscape. European Journal of Human Genetics, 13, 1293-1302. http://dx.doi.org/10.1038/sj.ejhg.5201482

Al-Zahery, N., Pala, M., Battaglia, V., Grugni, V., Hamod, M. A., Kashani, B. H. et al. (2011). In Search of the Genetic Footprints of Sumerians: A Survey of Y-Chromosome and mtDNA Variation in the Marsh Arabs of Iraq. BMC Evolutionary Biology, 11, 288. http://dx.doi.org/10.1186/1471-2148-11-288

Balanovsky, O., Rootsi, S., Pshenichnov, A., Kivisild, T., Churnosov, M., Evseeva, I. et al. (2008). Two Sources of the Russian Patrilineal Heritage in Their Eurasian Context. American Journal of Human Genetics, 82, 236-250. http://dx.doi.org/10.1016/j.ajhg.2007.09.019

Battaglia, V., Fornarino, S., Al-Zahery, N., Olivieri, A., Pala, M., Myres, N. M. et al.. (2009). Y-Chromosomal Evidence of the Cultural Diffusion of Agriculture in Southeast Europe. European Journal of Human Genetics, 17, 820-830. http://dx.doi.org/10.1038/ejhg.2008.249

Cadenas, A. M., Zhivotovsky, L. A., Cavalli-Sforza, L. L., Underhill, P. A., \& Herrera, R. J. (2008). Y-Chromosome Diversity Characterizes the Gulf of Oman. European Journal of Human Genetics, 16, 374-386. http://dx.doi.org/10.1038/sj.ejhg.5201934

Capelli, C., Brisighelli, F., Scarnicci, F., Arredi, B., Caglia, A., Vetrugno, G. et al. (2007). Y chromosome Genetic Variation in the Italian Peninsula Is Clinal and Supports an Admixture Model for the Mesolithic-Neolithic Encounter. Molecular Phylogenetics and Evolution, 44, 228-239. http://dx.doi.org/10.1016/j.ympev.2006.11.030

Capelli, C., Redhead, N., Abernethy, J. K., Gratrix, F., Wilson, J. F., Moen, T. et al. (2003). A Y Chromosome Census of the British Isles. Current Biology, 13, 979-984. http://dx.doi.org/10.1016/s0960-9822(03)00373-7

Cinnioğlu, C., King, R., Kivisild, T., Kalfoğlu, E., Atasoy, S., Cavalleri, G. L. Et al. (2004). Excavating Y-Chromosome Haplotype Strata in Anatolia. Human genetics, 114, 127-148. http://dx.doi.org/10.1007/s00439-003-1031-4

Derenko, M., Malyarchuk, B., Denisova, G., Wozniak, M., Grzybowski, T., Dambueva, I., \& Zakharov, I. (2007). Y-Chromosome Haplogroup N Dispersals from South Siberia to Europe. Journal of Human Genetics, 52, 763-770. http://dx.doi.org/10.1007/s10038-007-0179-5

Dulik, M. C., Osipova, L. P., \& Schurr, T. G. (2011). Y-Chromosome Variation in Altaian Kazakhs Reveals a Common Paternal Gene Pool for Kazakhs and the Influence of Mongolian Expansions. PLoS ONE, 6, e17548. http://dx.doi.org/10.1371/journal.pone.0017548

Flores, C., Maca-Meyer, N., Larruga, J. M., Cabrera, V. M., Karadsheh, N., \& Gonzalez, A. M. (2005). Isolates in a Corridor of Migrations: A High-Resolution Analysis of Y-Chromosome Variation in Jordan. Journal of Human Genetics, 50, 435441. http://dx.doi.org/10.1007/s10038-005-0274-4

Francalacci, P., Morelli, L., Underhill, P. A., Lillie, A. S., Passarino, G., Useli, A. et al. (2003). Peopling of Three Mediterranean Islands (Corsica, Sardinia, and Sicily) Inferred by Y-Chromosome Biallelic Variability. American Journal of Physical Anthropology, 121, 270-279. http://dx.doi.org/10.1002/ajpa.10265

Hammer, M. F., Karafet, T. M., Park, H., Omoto, K., Harihara, S., Stoneking, M., \& Horai, S. (2005). Dual Origins of the 
Japanese: Common Ground for Hunter-Gatherer and Farmer Y Chromosomes. Journal of Human Genetics, 51, 47-58. http://dx.doi.org/10.1007/s10038-005-0322-0

Karachanak, S., Grugni, V., Fornarino, S., Nesheva, D., Al-Zahery, N., Battaglia, V. et al. (2013). Y-Chromosome Diversity in Modern Bulgarians: New Clues about Their Ancestry. PLoS ONE, 8, e56779.

http://dx.doi.org/10.1371/journal.pone.0056779

Karlsson, A. O., Wallerström, T., Götherström, A., \& Holmlund, G. (2006). Y-Chromosome Diversity in Sweden-A Long-Time Perspective. European Journal of Human Genetics, 14, 963-970. http://dx.doi.org/10.1038/sj.ejhg.5201651

King, R., Özcan, S., Carter, T., Kalfoğlu, E., Atasoy, S., Triantaphyllidis, C. et al. (2008). Differential Y-Chromosome Anatolian Influences on the Greek and Cretan Neolithic. Annals of Human Genetics, 72, 205-214. http://dx.doi.org/10.1111/j.1469-1809.2007.00414.x

Kushniarevich, A., Sivitskaya, L., Danilenko, N., Novogrodskii, T., Tsybovsky, I., Kiseleva, A. et al. (2013). Uniparental Genetic Heritage of Belarusians: Encounter of Rare Middle Eastern Matrilineages with a Central European Mitochondrial DNA Pool. PLoS ONE, 8, e66499. http://dx.doi.org/10.1371/journal.pone.0066499

Lacau, H., Gayden, T., Regueiro, M., Chennakrishnaiah, S., Bukhari, A., Underhill, P. A. et al. (2012). Afghanistan from a Y-Chromosome Perspective. European Journal of Human Genetics, 20, 1063-1070. http://dx.doi.org/10.1038/ejhg.2012.59

Larmuseau, M. H., Vanderheyden, N., Jacobs, M., Coomans, M., Larno, L., \& Decorte, R. (2011). Micro-Geographic Distribution of Y-Chromosomal Variation in the Central-Western European Region Brabant. Forensic Science International: Genetics, 5, 95-99. http://dx.doi.org/10.1016/j.fsigen.2010.08.020

López-Parra, A., Gusmao, L., Tavares, L., Baeza, C., Amorim, A., Mesa, M. et al. (2009). In Search of the Pre- and Post-Neolithic Genetic Substrates in Iberia: Evidence from Y-Chromosome in Pyrenean Populations. Annals of Human Genetics, 73, 42-53. http://dx.doi.org/10.1111/j.1469-1809.2008.00478.x

Luis, J. R., Rowold, D. J., Regueiro, M., Caeiro, B., Cinnioğlu, C., Roseman, C. et al. (2004). The Levant versus the Horn of Africa: Evidence for Bidirectional Corridors of Human Migrations. The American Journal of Human Genetics, 74, 532544. http://dx.doi.org/10.1086/382286

Martinez, L., Underhill, P. A., Zhivotovsky, L. A., Gayden, T., Moschonas, N. K., Chow, C.-E. T. et al. (2007). Paleolithic Y-Haplogroup Heritage Predominates in a Cretan Highland Plateau. European Journal of Human Genetics, 15, 485-493. http://www.nature.com/ejhg/journal/v15/n4/suppinfo/5201769s1.html http://dx.doi.org/10.1038/sj.ejhg.5201769

Martinez-Cruz, B., Ioana, M., Calafell, F., Arauna, L. R., Sanz, P., Ionescu, R. et al. (2012). Y-Chromosome Analysis in Individuals Bearing the Basarab Name of the First Dynasty of Wallachian Kings. PLoS ONE, 7, e41803. http://dx.doi.org/10.1371/journal.pone.0041803

Mirabal, S., Regueiro, M., Cadenas, A. M., Cavalli-Sforza, L. L., Underhill, P. A., Verbenko, D. A. et al. (2009). YChromosome Distribution within the Geo-Linguistic landscape of Northwestern Russia. European Journal of Human Genetics, 17, 1260-1273. http://www.nature.com/ejhg/journal/v17/n10/suppinfo/ejhg20096s1.html http://dx.doi.org/10.1038/ejhg.2009.6

Noveski, P., Trivodalieva, S., Efremov, G., \& Plaseska-Karanfilska, D. (2010). Y Chromosome Single Nucleotide Polymorphisms Typing by SNaPshot Minisequencing. Balkan Journal of Medical Genetics, 13, 9-16. http://dx.doi.org/10.2478/v10034-010-0013-9

Regueiro, M., Cadenas, A., Gayden, T., Underhill, P., \& Herrera, R. (2006). Iran: Tricontinental Nexus for Y-Chromosome Driven Migration. Human Heredity, 61, 132-143. http://dx.doi.org/10.1159/000093774

Regueiro, M., Rivera, L., Damnjanovic, T., Lukovic, L., Milasin, J., \& Herrera, R. J. (2012). High Levels of Paleolithic Y-Chromosome Lineages Characterize Serbia. Gene, 498, 59-67. http://dx.doi.org/10.1016/j.gene.2012.01.030

Semino, O., Passarino, G., Oefner, P. J., Lin, A. A., Arbuzova, S., Beckman, L. E. et al. (2000). The Genetic Legacy of Paleolithic Homo Sapiens Sapiens in Extant Europeans: A Y Chromosome Perspective. Science, 290, 1155-1159. http://dx.doi.org/10.1126/science.290.5494.1155

Sengupta, S., Zhivotovsky, L. A., King, R., Mehdi, S., Edmonds, C. A., Chow, C.-E. T. et al. (2006). Polarity and Temporality of High-Resolution Y-Chromosome Distributions in India Identify Both Indigenous and Exogenous Expansions and Reveal Minor Genetic Influence of Central Asian Pastoralists. The American Journal of Human Genetics, 78, 202221. http://dx.doi.org/10.1086/499411

Varzari, A., Kharkov, V., Nikitin, A. G., Raicu, F., Simonova, K., Stephan, W. et al. (2013). Paleo-Balkan and Slavic Contributions to the Genetic Pool of Moldavians: Insights from the Y Chromosome. PLoS ONE, 8, e53731. http://dx.doi.org/10.1371/journal.pone.0053731

Zhong, H., Shi, H., Qi, X.-B., Duan, Z.-Y., Tan, P.-P., Jin, L. et al. (2011). Extended Y Chromosome Investigation Suggests Postglacial Migrations of Modern Humans into East Asia via the Northern Route. Molecular Biology and Evolution, 28, 717-727. http://dx.doi.org/10.1093/molbev/msq247 
(c)

\begin{tabular}{|c|c|c|c|c|}
\hline Country & Reference & $\mathrm{N}$ & $\mathrm{Hg} \mathrm{Q}$ & Hg Q \% \\
\hline Afghanistan-North & Lacau et al., 2012 & 44 & 2 & 4.55 \\
\hline Afghanistan-South & Lacau et al., 2012 & 146 & 4 & 2.74 \\
\hline Albania & Battaglia et al., 2009 & 55 & 0 & 0 \\
\hline Altai & Hammer et al, 2005 & 98 & 17 & 17.35 \\
\hline Altaian Kazakhs (Southeastern) & Dulik et al., 2011 & 89 & 0 & 0 \\
\hline Altaian Kazakhs (Southwestern) & Dulik et al., 2011 & 30 & 1 & 3.33 \\
\hline Anhui & Zhong et al., 2011 & 52 & 4 & 7.69 \\
\hline Antwerpen & Larmuseau et al., 2011 & 72 & 0 & 0 \\
\hline Balkarians & Battaglia et al., 2009 & 38 & 0 & 0 \\
\hline Belarussian-East Polesie & Kushniarevich et al., 2013 & 96 & 0 & 0 \\
\hline Belarussian-West Polesie & Kushniarevich et al., 2013 & 121 & 1 & 0.83 \\
\hline Belarussian-Centre & Kushniarevich et al., 2013 & 88 & 0 & 0 \\
\hline Belarussian-East & Kushniarevich et al., 2013 & 86 & 0 & 0 \\
\hline Belarussian-North & Kushniarevich et al., 2013 & 101 & 0 & 0 \\
\hline Belarussian-West & Kushniarevich et al., 2013 & 73 & 0 & 0 \\
\hline Bosniacs & Battaglia et al., 2009 & 84 & 0 & 0 \\
\hline Bosnian Croats & Battaglia et al., 2009 & 90 & 0 & 0 \\
\hline Bosnian Serbs & Battaglia et al., 2009 & 81 & 0 & 0 \\
\hline Bulgaria & Karachanak et al., 2013 & 808 & 4 & 0.50 \\
\hline Buryat & Hammer et al, 2005 & 81 & 0 & 0 \\
\hline Cambodia & Sengupta et al., 2006 & 6 & 0 & 0 \\
\hline Chechen (Chechnya) & Balanovsky et al., 2011 & 112 & 4 & 3.57 \\
\hline Chechen (Dagestan) & Balanovsky et al., 2011 & 118 & 0 & 0 \\
\hline Chechen (Ingushetia) & Balanovsky et al., 2011 & 100 & 7 & 7.00 \\
\hline China-Dai & Sengupta et al., 2006 & 7 & 0 & 0 \\
\hline China-Daur & Sengupta et al., 2006 & 7 & 0 & 0 \\
\hline China-Hezhen & Sengupta et al., 2006 & 6 & 0 & 0 \\
\hline China-Lahu & Sengupta et al., 2006 & 7 & 0 & 0 \\
\hline China-Naxi & Sengupta et al., 2006 & 8 & 0 & 0 \\
\hline China-Oroqen & Sengupta et al., 2006 & 7 & 0 & 0 \\
\hline China-Tu & Sengupta et al., 2006 & 7 & 0 & 0 \\
\hline China-Tujia & Sengupta et al., 2006 & 9 & 0 & 0 \\
\hline China-Uygur & Sengupta et al., 2006 & 8 & 0 & 0 \\
\hline China-Xibo & Sengupta et al., 2006 & 8 & 0 & 0 \\
\hline China-Yi & Sengupta et al., 2006 & 9 & 0 & 0 \\
\hline Chongqing & Zhong et al., 2011 & 43 & 3 & 6.98 \\
\hline
\end{tabular}




\section{Continued}

\begin{tabular}{|c|c|c|c|c|}
\hline Corsica & Francalacci et al., 2003 & 34 & 0 & 0 \\
\hline Crete & Martinez et al., 2007; King et al., 2008 & 361 & 2 & 0.55 \\
\hline Croats & Battaglia et al., 2009 & 118 & 0 & 0 \\
\hline Czechs & Battaglia et al., 2009 & 75 & 0 & 0 \\
\hline Even & Hammer et al., 2005 & 31 & 1 & 3.23 \\
\hline Evenk & Hammer et al., 2005 & 95 & 4 & 4.21 \\
\hline France & Semino et al., 2000 & 23 & 0 & 0 \\
\hline Fujian & Zhong et al., 2011 & 112 & 0 & 0 \\
\hline FYROM & Noveski et al., 2010 & 211 & 0 & 0 \\
\hline Gansu & Zhong et al., 2011 & 221 & 12 & 5.43 \\
\hline Georgians & Battaglia et al., 2009 & 66 & 0 & 0 \\
\hline Germany & Semino et al., 2000 & 16 & 0 & 0 \\
\hline Gipuzkoa Basques & Alonso et al., 2005 & 74 & 0 & 0 \\
\hline Greece & Battaglia et al., 2009 & 92 & 0 & 0 \\
\hline Greece-Nea Nikomedeia & King et al., 2008 & 57 & 0 & 0 \\
\hline Greece-Sesklo & King et al., 2008 & 57 & 0 & 0 \\
\hline Guangdong & Zhong et al., 2011 & 91 & 0 & 0 \\
\hline Guangxi & Zhong et al., 2011 & 272 & 2 & 0.74 \\
\hline Guizhou & Zhong et al., 2011 & 533 & 5 & 0.94 \\
\hline Hainan & Zhong et al., 2011 & 110 & 0 & 0 \\
\hline Heilongjiang & Zhong et al., 2011 & 171 & 3 & 1.75 \\
\hline Henan & Zhong et al., 2011 & 66 & 3 & 4.55 \\
\hline Hubei & Zhong et al., 2011 & 83 & 0 & 0 \\
\hline Hunan & Zhong et al., 2011 & 133 & 4 & 3.01 \\
\hline Hungary & Battaglia et al., 2009 & 53 & 0 & 0 \\
\hline India North & Sengupta et al., 2006 & 80 & 1 & 1.25 \\
\hline India-Northeast & Sengupta et al., 2006 & 87 & 0 & 0 \\
\hline India-Central & Sengupta et al., 2006 & 71 & 1 & 1.41 \\
\hline India-East & Sengupta et al., 2006 & 128 & 0 & 0 \\
\hline India-South & Sengupta et al., 2006 & 303 & 1 & 0.33 \\
\hline India-West & Sengupta et al., 2006 & 59 & 0 & 0 \\
\hline Indonesia-East & Hammer et al, 2005 & 55 & 0 & 0 \\
\hline Indonesia-West & Hammer et al, 2005 & 25 & 0 & 0 \\
\hline Inner Mongolia & Zhong et al., 2011 & 53 & 1 & 1.89 \\
\hline Iran & Grugni et al., 2012 & 827 & 47 & 5.68 \\
\hline Iran North & Regueiro et al., 2006 & 33 & 3 & 9.09 \\
\hline Iran South & Regueiro et al., 2006 & 117 & 3 & 2.56 \\
\hline
\end{tabular}




\section{Continued}

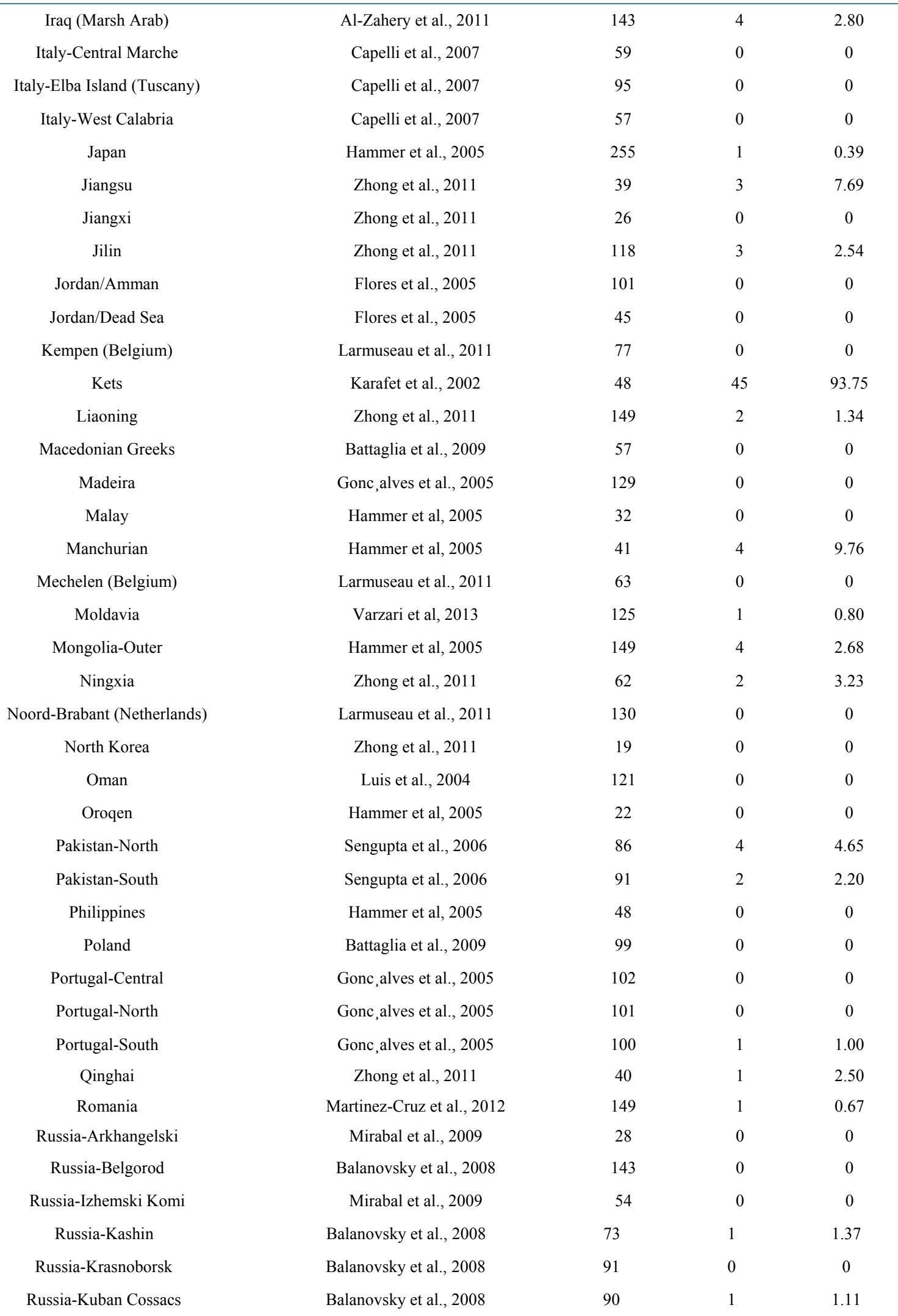




\section{Continued}

Russia-Kursk

Russia-Livni

Russia-Mezen

Russia-Ostrov

Russia-Pinega

Russia-Porhov

Russia-Priluzski Komi

Russia-Pristen

Russia-Pskov

Russia-Repievka

Russia-Roslavl

Russia-Siberia (Khanty)

Russia-Tver

Russia-Unzha

Russia-Vologda

Sardinia

Selkups

Serbia

Shandong

Shanghai

Shannxi

Shanxi

Sichuan

Sicily

Slovenia

South Korea

Spain-Alto Urgel

Spain-Cerda na

Spain-Cinco Villas

Spain-Jacetania

Spain-Vallede Ar'an

Swedish Saami

Taiwan

Tibet

Tibetans

Turkey-central

Turkey-east

Turkey-west

Ukraine

Vietnam
Mirabal et al., 2009

Balanovsky et al., 2008

Balanovsky et al., 2008

Balanovsky et al., 2008

Balanovsky et al., 2008

Balanovsky et al., 2008

Mirabal et al., 2009

Balanovsky et al., 2008

Balanovsky et al., 2008

Balanovsky et al., 2008

Balanovsky et al., 2008

Mirabal et al., 2009

Mirabal et al., 2009

Balanovsky et al., 2008

Balanovsky et al., 2008

Francalacci et al., 2003

Karafet et al., 2002

Regueiro et al., 2012

Zhong et al., 2011

Zhong et al., 2011

Zhong et al., 2011

Zhong et al., 2011

Zhong et al., 2011

Francalacci et al., 2003

Battaglia et al., 2009

Hammer et al, 2005

Lopez-Parra et al., 2009

Lopez-Parra et al., 2009

Lopez-Parra et al., 2009

Lopez-Parra et al., 2009

Lopez-Parra et al., 2009

Karlsson et al., 2006

Hammer et al., 2005

Hammer et al., 2005

Zhong et al., 2011

Cinnioglu et al., 2004

Cinnioglu et al., 2004

Cinnioglu et al., 2004

Battaglia et al., 2009

Hammer et al., 2005

\begin{tabular}{|c|c|c|}
\hline 40 & 0 & 0 \\
\hline 110 & 1 & 0.91 \\
\hline 54 & 0 & 0 \\
\hline 75 & 0 & 0 \\
\hline 114 & 1 & 0.88 \\
\hline 57 & 0 & 0 \\
\hline 49 & 0 & 0 \\
\hline 45 & 0 & 0 \\
\hline 132 & 0 & 0 \\
\hline 96 & 0 & 0 \\
\hline 107 & 0 & 0 \\
\hline 27 & 1 & 3.70 \\
\hline 38 & 0 & 0 \\
\hline 52 & 0 & 0 \\
\hline 121 & 2 & 1.65 \\
\hline 78 & 0 & 0 \\
\hline 131 & 87 & 66.41 \\
\hline 103 & 0 & 0 \\
\hline 115 & 2 & 1.74 \\
\hline 17 & 0 & 0 \\
\hline 56 & 1 & 1.79 \\
\hline 56 & 3 & 5.36 \\
\hline 113 & 1 & 0.88 \\
\hline 51 & 0 & 0 \\
\hline 75 & 0 & 0 \\
\hline 75 & 0 & 0 \\
\hline 34 & 0 & 0 \\
\hline 37 & 0 & 0 \\
\hline 42 & 0 & 0 \\
\hline 31 & 0 & 0 \\
\hline 25 & 0 & 0 \\
\hline 38 & 0 & 0 \\
\hline 132 & 0 & 0 \\
\hline 105 & 0 & 0 \\
\hline 222 & 1 & 0.45 \\
\hline 152 & 3 & 1.97 \\
\hline 208 & 6 & 2.88 \\
\hline 163 & 1 & 0.61 \\
\hline 92 & 1 & 1.09 \\
\hline 70 & 5 & 7.14 \\
\hline
\end{tabular}




\begin{tabular}{rcccc} 
Continued & & & & \\
\hline Vlaams and Waals Brabant (Belgium) & Larmuseau et al., 2011 & 135 & 0 & 0 \\
Xingjiang & Zhong et al., 2011 & 244 & 11 & 4.51 \\
Xinjiang & Zhong et al., 2011 & 61 & 0 & 0 \\
Yakut & Sengupta et al., 2006 & 18 & 0 & 0 \\
Yemen & Cadenas et al., 2008 & 62 & 0 & 0 \\
Yunnan & Zhong et al., 2011 & 447 & 7 & 1.57 \\
Zhejiang & Zhong et al., 2011 & 102 & 2 & 1.96 \\
\hline
\end{tabular}

\section{References S1(c)}

Alonso, S., Flores, C., Cabrera, V., Alonso, A., Martin, P., Albarran, C. et al. (2005). The Place of the Basques in the European Y-Chromosome Diversity Landscape. European Journal of Human Genetics, 13, 1293-1302.

http://dx.doi.org/10.1038/sj.ejhg.5201482

Al-Zahery, N., Pala, M., Battaglia, V., Grugni, V., Hamod, M. A., Kashani, B. H. et al. (2011). In Search of the Genetic Footprints of Sumerians: A Survey of Y-Chromosome and mtDNA Variation in the Marsh Arabs of Iraq. BMC Evolutionary Biology, 11, 288. http://dx.doi.org/10.1186/1471-2148-11-288

Balanovsky, O., Dibirova, K., Dybo, A., Mudrak, O., Frolova, S., Pocheshkhova, E. et al. (2011). Parallel Evolution of Genes and Languages in the Caucasus Region. Molecular Biology and Evolution, 28, 2905-2920. http://dx.doi.org/10.1093/molbev/msr126

Balanovsky, O., Rootsi, S., Pshenichnov, A., Kivisild, T., Churnosov, M., Evseeva, I. et al. (2008). Two Sources of the Russian Patrilineal Heritage in Their Eurasian Context. American Journal of Human Genetics, 82, 236-250. http://dx.doi.org/10.1016/j.ajhg.2007.09.019

Battaglia, V., Fornarino, S., Al-Zahery, N., Olivieri, A., Pala, M., Myres, N. M. et al.. (2009). Y-Chromosomal Evidence of the Cultural Diffusion of Agriculture in Southeast Europe. European Journal of Human Genetics, 17, 820-830. http://dx.doi.org/10.1038/ejhg.2008.249

Cadenas, A. M., Zhivotovsky, L. A., Cavalli-Sforza, L. L., Underhill, P. A., \& Herrera, R. J. (2008). Y-Chromosome Diversity Characterizes the Gulf of Oman. European Journal of Human Genetics, 16, 374-386. http://dx.doi.org/10.1038/sj.ejhg.5201934

Capelli, C., Brisighelli, F., Scarnicci, F., Arredi, B., Caglia, A., Vetrugno, G. et al. (2007). Y Chromosome Genetic Variation in the Italian Peninsula Is Clinal and Supports an Admixture Model for the Mesolithic-Neolithic Encounter. Molecular Phylogenetics and Evolution, 44, 228-239. http://dx.doi.org/10.1016/j.ympev.2006.11.030

Cinnioğlu, C., King, R., Kivisild, T., Kalfoğlu, E., Atasoy, S., Cavalleri, G. L. et al. (2004). Excavating Y-Chromosome Haplotype Strata in Anatolia. Human genetics, 114, 127-148. http://dx.doi.org/10.1007/s00439-003-1031-4

Dulik, M. C., Osipova, L. P., \& Schurr, T. G. (2011). Y-Chromosome Variation in Altaian Kazakhs Reveals a Common Paternal Gene Pool for Kazakhs and the Influence of Mongolian Expansions. PLoS ONE, 6, e17548. http://dx.doi.org/10.1371/journal.pone.0017548

Flores, C., Maca-Meyer, N., Larruga, J. M., Cabrera, V. M., Karadsheh, N., \& Gonzalez, A. M. (2005). Isolates in a Corridor of Migrations: A High-Resolution Analysis of Y-Chromosome Variation in Jordan. Journal of Human Genetics, 50, 435441. http://dx.doi.org/10.1007/s10038-005-0274-4

Francalacci, P., Morelli, L., Underhill, P. A., Lillie, A. S., Passarino, G., Useli, A. et al. (2003). Peopling of Three Mediterranean Islands (Corsica, Sardinia, and Sicily) inferred by Y-Chromosome Biallelic Variability. American Journal of Physical Anthropology, 121, 270-279. http://dx.doi.org/10.1002/ajpa.10265

Gonçalves, R., Freitas, A., Branco, M., Rosa, A., Fernandes, A. T., Zhivotovsky, L. A. et al. (2005). Y-Chromosome Lineages from Portugal, Madeira and Açores Record Elements of Sephardim and Berber Ancestry. Annals of Human Genetics, 69, 443-454. http://dx.doi.org/10.1111/j.1529-8817.2005.00161.x

Grugni, V., Battaglia, V., Hooshiar Kashani, B., Parolo, S., Al-Zahery, N., Achilli, A. et al. (2012). Ancient Migratory Events in the Middle East: New Clues from the Y-Chromosome Variation of Modern Iranians. PLoS ONE, 7, e41252. http://dx.doi.org/10.1371/journal.pone.0041252

Hammer, M. F., Karafet, T. M., Park, H., Omoto, K., Harihara, S., Stoneking, M., \& Horai, S. (2005). Dual Origins of the Japanese: Common Ground for Hunter-Gatherer and Farmer Y Chromosomes. Journal of Human Genetics, 51, 47-58. http://dx.doi.org/10.1007/s10038-005-0322-0

Karachanak, S., Grugni, V., Fornarino, S., Nesheva, D., Al-Zahery, N., Battaglia, V. et al. (2013). Y-Chromosome Diversity in Modern Bulgarians: New Clues about Their Ancestry. PLoS ONE, 8, e56779. 
http://dx.doi.org/10.1371/journal.pone.0056779

Karafet, T. M., Osipova, L. P., Gubina, M. A., Posukh, O. L., Zegura, S. L., \& Hammer, M. F. (2002). High Levels of Y-Chromosome Differentiation among Native Siberian Populations and the Genetic Signature of a Boreal HunterGatherer Way of Life. Human Biology, 74, 761-789. http://dx.doi.org/10.1353/hub.2003.0006

Karlsson, A. O., Wallerström, T., Götherström, A., \& Holmlund, G. (2006). Y-Chromosome Diversity in Sweden-A Long-Time Perspective. European Journal of Human Genetics, 14, 963-970. http://dx.doi.org/10.1038/sj.ejhg.5201651

King, R., Özcan, S., Carter, T., Kalfoğlu, E., Atasoy, S., Triantaphyllidis, C. et al. (2008). Differential Y-Chromosome Anatolian Influences on the Greek and Cretan Neolithic. Annals of Human Genetics, 72, 205-214. http://dx.doi.org/10.1111/j.1469-1809.2007.00414.x

Kushniarevich, A., Sivitskaya, L., Danilenko, N., Novogrodskii, T., Tsybovsky, I., Kiseleva, A. et al. (2013). Uniparental Genetic Heritage of Belarusians: Encounter of Rare Middle Eastern Matrilineages with a Central European Mitochondrial DNA Pool. PLoS ONE, 8, e66499. http://dx.doi.org/10.1371/journal.pone.0066499

Lacau, H., Gayden, T., Regueiro, M., Chennakrishnaiah, S., Bukhari, A., Underhill, P. A. et al. (2012). Afghanistan from a Y-Chromosome Perspective. European Journal of Human Genetics, 20, 1063-1070. http://dx.doi.org/10.1038/ejhg.2012.59

Larmuseau, M. H., Vanderheyden, N., Jacobs, M., Coomans, M., Larno, L., \& Decorte, R. (2011). Micro-Geographic Distribution of Y-Chromosomal Variation in the Central-Western European Region Brabant. Forensic Science International: Genetics, 5, 95-99. http://dx.doi.org/10.1016/j.fsigen.2010.08.020

López-Parra, A., Gusmao, L., Tavares, L., Baeza, C., Amorim, A., Mesa, M. et al. (2009). In Search of the Pre- and Post-Neolithic Genetic Substrates in Iberia: Evidence from Y-Chromosome in Pyrenean Populations. Annals of Human Genetics, 73, 42-53. http://dx.doi.org/10.1111/j.1469-1809.2008.00478.x

Luis, J. R., Rowold, D. J., Regueiro, M., Caeiro, B., Cinnioğlu, C., Roseman, C. et al. (2004). The Levant versus the Horn of Africa: Evidence for Bidirectional Corridors of Human Migrations. The American Journal of Human Genetics, 74, 532544. http://dx.doi.org/10.1086/382286

Martinez, L., Underhill, P. A., Zhivotovsky, L. A., Gayden, T., Moschonas, N. K., Chow, C.-E. T. et al. (2007). Paleolithic Y-Haplogroup Heritage Predominates in a Cretan Highland Plateau. European Journal of Human Genetics, 15, 485-493. http://www.nature.com/ejhg/journal/v15/n4/suppinfo/5201769s1.html http://dx.doi.org/10.1038/sj.ejhg.5201769

Martinez-Cruz, B., Ioana, M., Calafell, F., Arauna, L. R., Sanz, P., Ionescu, R. et al. (2012). Y-Chromosome Analysis in Individuals Bearing the Basarab Name of the First Dynasty of Wallachian Kings. PLoS ONE, 7, e41803. http://dx.doi.org/10.1371/journal.pone.0041803

Mirabal, S., Regueiro, M., Cadenas, A. M., Cavalli-Sforza, L. L., Underhill, P. A., Verbenko, D. A. et al. (2009). Y-Chromosome Distribution within the Geo-Linguistic Landscape of Northwestern Russia. European Journal of Human Genetics, 17, 1260-1273. http://www.nature.com/ejhg/journal/v17/n10/suppinfo/ejhg20096s1.html http://dx.doi.org/10.1038/ejhg.2009.6

Noveski, P., Trivodalieva, S., Efremov, G., \& Plaseska-Karanfilska, D. (2010). Y Chromosome Single Nucleotide Polymorphisms Typing by SNaPshot Minisequencing. Balkan Journal of Medical Genetics, 13, 9-16. http://dx.doi.org/10.2478/v10034-010-0013-9

Regueiro, M., Cadenas, A., Gayden, T., Underhill, P., \& Herrera, R. (2006). Iran: Tricontinental Nexus for Y-Chromosome Driven Migration. Human Heredity, 61, 132-143. http://dx.doi.org/10.1159/000093774

Regueiro, M., Rivera, L., Damnjanovic, T., Lukovic, L., Milasin, J., \& Herrera, R. J. (2012). High Levels of Paleolithic Y-Chromosome Lineages Characterize Serbia. Gene, 498, 59-67. http://dx.doi.org/10.1016/j.gene.2012.01.030

Semino, O., Passarino, G., Oefner, P. J., Lin, A. A., Arbuzova, S., Beckman, L. E. et al. (2000). The Genetic Legacy of Paleolithic Homo Sapiens Sapiens in Extant Europeans: A Y Chromosome Perspective. Science, 290, 1155-1159. http://dx.doi.org/10.1126/science.290.5494.1155

Sengupta, S., Zhivotovsky, L. A., King, R., Mehdi, S., Edmonds, C. A., Chow, C.-E. T. et al. (2006). Polarity and Temporality of High-Resolution Y-Chromosome Distributions in India Identify Both Indigenous and Exogenous Expansions and Reveal Minor Genetic Influence of Central Asian Pastoralists. The American Journal of Human Genetics, 78, 202-221. http://dx.doi.org/10.1086/499411

Varzari, A., Kharkov, V., Nikitin, A. G., Raicu, F., Simonova, K., Stephan, W. et al. (2013). Paleo-Balkan and Slavic Contributions to the Genetic Pool of Moldavians: Insights from the Y Chromosome. PLoS ONE, 8, e53731. http://dx.doi.org/10.1371/journal.pone.0053731

Zhong, H., Shi, H., Qi, X.-B., Duan, Z.-Y., Tan, P.-P., Jin, L. et al. (2011). Extended Y Chromosome Investigation Suggests Postglacial Migrations of Modern Humans into East Asia via the Northern Route. Molecular Biology and Evolution, 28, 717-727. http://dx.doi.org/10.1093/molbev/msq247 
Table S2. Absolute and percent frequencies of mtDNA haplogroup C (a), D (b) and Z (c) used to construct spatial frequency maps. N-number of analyzed individuals.

(a)

\begin{tabular}{|c|c|c|c|c|}
\hline Country & Reference & $\mathrm{N}$ & $\mathrm{Hg} \mathrm{C}$ & $\mathrm{Hg} \mathrm{C} \mathrm{( \% )}$ \\
\hline Adam & Grosheva et al., 2014 & 54 & 2 & 3.70 \\
\hline Afghanistan & Irwin et al., 2010 & 98 & 2 & 2.04 \\
\hline Ainu & Derenko et al., 2010 & 51 & 0 & 0 \\
\hline Altaian Kazakhs & Derenko et al., 2012 & 98 & 8 & 8.16 \\
\hline Altaians & Derenko et al., 2010 & 110 & 21 & 19.09 \\
\hline Ambon & Simonson et al., 2011 & 43 & 0 & 0 \\
\hline Asian Eskimos & Gubina et al., 2013 & 40 & 0 & 0 \\
\hline Austrians & Brandsta"tter et al., 2007 & 273 & 1 & 0.37 \\
\hline Azerbaijanians & Derenko et al., 2010 & 89 & 3 & 3.37 \\
\hline Azeris & Derenko et al., 2013 & 22 & 1 & 5 \\
\hline Bai & Liu et al., 2011 & 68 & 2 & 3 \\
\hline Bali & Simonson et al., 2011 & 82 & 0 & 0 \\
\hline Baluch & Quintana-Murci et al., 2004 & 39 & 0 & 0 \\
\hline Barghuts & Derenko et al., 2012 & 149 & 30 & 20 \\
\hline Bashkirs & Derenko et al., 2010 & 207 & 25 & 12 \\
\hline Basque Country & Prieto et al., 2011 & 29 & 0 & 0 \\
\hline Basques from Northern Navarre & Cardoso et al., 2011 & 110 & 0 & 0 \\
\hline Belarusians & Kushniarevich et al., 2013 & 267 & 3 & 1 \\
\hline Borneo & Simonson et al., 2011 & 157 & 2 & 1 \\
\hline Bosnians & Malyarchuk et al., 2003 & 144 & 0 & 0 \\
\hline Brahui & Quintana-Murci et al., 2004 & 38 & 0 & 0 \\
\hline British people & Zheng et al., 2012 & 94 & 0 & 0 \\
\hline Bukharan Arabs & Comas et al., 2004 & 20 & 4 & 20.00 \\
\hline Bulgarians & Karachanak et al., 2012 & 855 & 2 & 0.23 \\
\hline Buryats & Derenko et al., 2010 & 419 & 89 & 21 \\
\hline Cantabria & Álvarez-Iglesias et al., 2009 & 135 & 0 & 0 \\
\hline Catalonia & Álvarez-Iglesias et al., 2009 & 101 & 0 & 0 \\
\hline Central Portugal & Prieto et al., 2011 & 53 & 0 & 0 \\
\hline Chaplin & Volodko et al., 2008 & 50 & 0 & 0 \\
\hline Chechens & Derenko et al., 2010 & 67 & 3 & 4 \\
\hline
\end{tabular}




\section{Continued}

\begin{tabular}{|c|c|c|c|c|}
\hline Chinese & Derenko et al., 2010 & 1930 & 87 & 4.51 \\
\hline Chukchi & Derenko et al., 2010 & 417 & 55 & 13 \\
\hline Chukchi & Gubina et al., 2013 & 159 & 22 & 13.84 \\
\hline Chukchi & Volodko et al., 2008 & 182 & 34 & 18.80 \\
\hline Chutyr' & Grosheva et al., 2014 & 63 & 3 & 4.76 \\
\hline Chuvantsi & Derenko et al., 2010 & 32 & 10 & 31 \\
\hline Chuvashis & Derenko et al., 2010 & 142 & 2 & 1.41 \\
\hline Crimean Tatars & Comas et al., 2004 & 20 & 0 & 0 \\
\hline Cypriots & Irwin et al., 2008 & 91 & 0 & 0 \\
\hline Czech & Mielnik-Sikorska et al., 2013 & 85 & 0 & 0 \\
\hline Czechs (Western Bohemia) & Malyarchuk et al., 2006 & 179 & 0 & 0 \\
\hline Dai & Liu et al., 2011 & 41 & 5 & 12.20 \\
\hline Danes & Mikkelsen et al., 2010 & 201 & 0 & 0 \\
\hline Darginians & Derenko et al., 2010 & 37 & 1 & 2.70 \\
\hline Daurs, China & Derenko et al., 2010 & 45 & 3 & 6.67 \\
\hline Dolgans & Derenko et al., 2010 & 157 & 61 & 38.85 \\
\hline Dong & Liu et al., 2011 & 28 & 0 & 0 \\
\hline Dungan & Comas et al., 2004 & 16 & 1 & 6.25 \\
\hline East Evenks & Derenko et al., 2010 & 45 & 29 & 64 \\
\hline Eskimos & Derenko et al., 2010 & 254 & 2 & 0.79 \\
\hline European Russia (Novgorod oblast) & Grzybowski et al., 2007 & 157 & 0 & 0 \\
\hline Evenks & Derenko et al., 2010 & 71 & 51 & 72 \\
\hline Evenks & Gubina et al., 2013 & 38 & 20 & 53 \\
\hline Evenks, China & Derenko et al., 2010 & 47 & 9 & 19.15 \\
\hline Evens & Derenko et al., 2010 & 191 & 72 & 38 \\
\hline Ezhevo & Grosheva et al., 2014 & 39 & 11 & 28 \\
\hline Finns & Hedman et al., 2007 & 200 & 0 & 0 \\
\hline Fins & Zheng et al., 2012 & 97 & 0 & 0 \\
\hline French people & Richard et al., 2007 & 868 & 0 & 0 \\
\hline Gelao & Liu et al., 2011 & 177 & 14 & 8 \\
\hline Georgians & Derenko et al., 2010 & 57 & 1 & 1.75 \\
\hline Gilaki & Quintana-Murci et al., 2004 & 37 & 0 & 0 \\
\hline Gujarati & Quintana-Murci et al., 2004 & 34 & 0 & 0 \\
\hline
\end{tabular}




\section{Continued}

\begin{tabular}{|c|c|c|c|c|}
\hline Han Chinese & Yao et al., 2002 & 263 & 8 & 3.04 \\
\hline Hazara & Quintana-Murci et al., 2004 & 23 & 0 & 0 \\
\hline Hui & Yao et al., 2004 & 45 & 1 & 2.22 \\
\hline Hui, China & Derenko et al., 2010 & 45 & 1 & 2.22 \\
\hline Hungarians & Derenko et al., 2010 & 344 & 2 & 0.58 \\
\hline Hunza Burusho & Quintana-Murci et al., 2004 & 44 & 1 & 2 \\
\hline Hungarians (Budapest) & Irwin et al., 2007 & 211 & 0 & 0 \\
\hline Iranians & Derenko et al., 2013 & 352 & 5 & 1.42 \\
\hline Iranians & Comas et al., 2004 & 20 & 2 & 10.00 \\
\hline Iraqis & Al-Zahery et al., 2003 & 216 & 0 & 0 \\
\hline India & Derenko et al., 2010 & 2544 & 31 & 1.22 \\
\hline Itelmens & Derenko et al., 2010 & 46 & 6 & 13.04 \\
\hline Japanese & Derenko et al., 2010 & 1312 & 7 & 0.53 \\
\hline Java & Simonson et al., 2011 & 46 & 1 & 2 \\
\hline Kabachigurt & Grosheva et al., 2014 & 57 & 3 & 5 \\
\hline Kabardinians & Derenko et al., 2010 & 51 & 1 & 2 \\
\hline Kalash & Quintana-Murci et al., 2004 & 44 & 0 & 0 \\
\hline Kalmyks & Derenko et al., 2010 & 110 & 12 & 11 \\
\hline Karakalpaks & Derenko et al., 2010 & 108 & 16 & 15 \\
\hline Kazak & Comas et al., 2004 & 20 & 1 & 5 \\
\hline Kazak & Yao et al., 2004 & 53 & 7 & 13 \\
\hline Kazakhs & Derenko et al., 2010 & 511 & 44 & 9 \\
\hline Kazakhs, China & Derenko et al., 2010 & 53 & 7 & 13 \\
\hline Kazakhstan & Irwin et al., 2010 & 256 & 20 & 8 \\
\hline Kazan Tatars (Azmakaevo) & Malyarchuk et al., 2010 & 71 & 4 & 6 \\
\hline Kets & Derenko et al., 2010 & 104 & 13 & 13 \\
\hline Khakassians & Derenko et al., 2010 & 110 & 30 & 27 \\
\hline Khakassians & Derenko et al., 2007 & 57 & 11 & 19 \\
\hline Khamnigans & Derenko et al., 2010 & 99 & 16 & 16.16 \\
\hline Khants & Derenko et al., 2010 & 106 & 11 & 10.38 \\
\hline Khanty & Pimenoff et al., 2008 & 106 & 11 & 10 \\
\hline Khoremian Uzbek & Comas et al., 2004 & 20 & 0 & 0 \\
\hline Kirghiz & Derenko et al., 2010 & 200 & 28 & 14 \\
\hline
\end{tabular}




\section{Continued}

Komis

Koreans

Koreans, China

Koryaks

Koryaks

Kurdish

Kurdish-Western Iran

Kurdish-Turkmenistan

Kuzyumovo

Kyrgyz

Kyrgyzstan

Lahu

Lesagurt

Lombok

Lower Kolyma-Indigirka

Lur

Macedonians (FYROM)

Makrani

Manchu

Mansi

Mansi

Maris

Mazandarian

Miao

Mongolian

Mongolians

Mongolians, China

Mordvins

Mulam

Nanaians

Nanais

Naukan

Naxi

Negidals
Derenko et al., 2010

Derenko et al., 2010

Derenko et al., 2010

Derenko et al., 2010

Gubina et al., 2013

Derenko et al., 2010

Quintana-Murci et al., 2004

Quintana-Murci et al., 2004

Grosheva et al., 2014

Comas et al., 2004

Irwin et al., 2010

Liu et al., 2011

Grosheva et al., 2014

Simonson et al., 2011

Volodko et al., 2008

Quintana-Murci et al., 2004

Zimmermann et al., 2007

Quintana-Murci et al., 2004

Zhao et al., 2011

Derenko et al., 2010

Pimenoff et al., 2008

Derenko et al., 2010

Quintana-Murci et al., 2004

Liu et al., 2011

Yao et al., 2004

Derenko et al., 2010

Derenko et al., 2010

Derenko et al., 2010

Liu et al., 2011

Gubina et al., 2013

Derenko et al., 2010

Volodko et al., 2008

Liu et al., 2011

Derenko et al., 2010
121

1297

48

182

69

135

20

32

72

20

249

35

63

44

82

17

200

33

47

161

63

234

21

103

49

150

97

120

39

86

85

39

45

33
5

2.00

0

36.81

23.19

0.74

0

9.38

1

30

12.85

0

11

2

$54,038 \quad 65.90$

$0 \quad 0$

0

$0 \quad 0$

24.26

$30 \quad 19$

$13 \quad 20.63$

$\begin{array}{ll}2 & 0.85\end{array}$

$$
0
$$

0

9.71

10.20

5

15

8

1.67

0

9.30

9

0

8.89

15 


\section{Continued}

Nenets
Nenets Tundra
Nganasan
Nganasans
Nivkhs
Nivkhs
Nogays

North and Central Italians

North Portugal

Northern Caucasians

Northern Greeks

$\mathrm{Nu}$

Orochens, China

Oroks

Osettins

Pakistani

Parsis

Pathan

Persian

Persians

Persians

Phillipines

Poland (Kashubians)

Poland (Podhale)

Poland Northeastern

Poland Northern

Poland Southern

$$
\text { Poles }
$$

Qashqais

Romanians

Saudi Arabs

Selkups

Serbia-Vojvodina Province

Shors

Shugnan

Shugnan, Tajikistan

Shui

Derenko et al., 2010

Derenko et al., 2010

Volodko et al., 2008

Derenko et al., 2010

Derenko et al., 2010

Gubina et al., 2013

Derenko et al., 2010

Turchi et al., 2008

Prieto et al., 2011

Derenko et al., 2010

Irwin et al., 2008

Liu et al., 2011

Derenko et al., 2010

Derenko et al., 2010

Derenko et al., 2010

Derenko et al., 2010

Quintana-Murci et al., 2004

Quintana-Murci et al., 2004

Quintana-Murci et al., 2004

Derenko et al., 2010

Derenko et al., 2013

Simonson et al., 2011

Mielnik-Sikorska et al., 2013

Mielnik-Sikorska et al., 2013

Grzybowski et al., 2007

Grzybowski et al., 2007

Grzybowski et al., 2007

Derenko et al., 2010

Derenko et al., 2013

Derenko et al., 2010

Abu-Amero et al., 2008, 2007

Derenko et al., 2010

Zgonjanin et al., 2010

Derenko et al., 2010

Quintana-Murci et al., 2004

Derenko et al., 2010

Liu et al., 2011
58

70

39

78

56

17

206

395

187

199

319

30

44

61

123

189

18

31

$34 \quad 49$

$20,007 \quad 51$

26

33

0

0

12.14

25

0

$$
0
$$

4

0

$$
0
$$

30

11

3.25

2

0

0

0

0.77

518

181

61

203

201

73

253

87

947

112

360

553

120

104

82

44

44

30

25

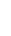

$44 \quad 0$

20

$\begin{array}{ll}4 & 0.77\end{array}$

0

$\begin{array}{ll}1 & 0.49\end{array}$

0

0

0

115

$\begin{array}{lll}9 & 1\end{array}$

2

\begin{tabular}{ll}
$10 \quad 2.78$ \\
\hline
\end{tabular}

00

$26 \quad 21.67$

040

$10 \quad 12$

$\begin{array}{ll}8 & 18\end{array}$

$\begin{array}{lll}8 & 18\end{array}$

0

0

9
1
3
0
0
14

0




\section{Continued}

\begin{tabular}{|c|c|c|c|c|}
\hline Sindhi & Quintana-Murci et al., 2004 & 23 & 0 & 0 \\
\hline Sireniki & Volodko et al., 2008 & 37 & 0 & 0 \\
\hline Slovaks & Lehocký et al., 2008 & 374 & 4 & 1.07 \\
\hline Slovenians & Malyarchuk et al., 2003 & 104 & 0 & 0 \\
\hline Sojots & Derenko et al., 2010 & 30 & 6 & 20.00 \\
\hline Southern Germany (Ulm) & Brandsta"tter et al., 2006 & 100 & 0 & 0 \\
\hline Spain & Prieto et al., 2011 & 403 & 0 & 0 \\
\hline Staraya Salya & Grosheva et al., 2014 & 54 & 4 & 7.41 \\
\hline Sulawesi & Simonson et al., 2011 & 237 & 1 & 0.42 \\
\hline Sumatra & Simonson et al., 2011 & 180 & 0 & 0 \\
\hline Sumba & Simonson et al., 2011 & 50 & 0 & 0 \\
\hline Svetozarevo & Grosheva et al., 2014 & 45 & 1 & 2 \\
\hline Taiwan & Simonson et al., 2011 & 78 & 0 & 0 \\
\hline Tajikistan & Irwin et al., 2010 & 244 & 36 & 14.75 \\
\hline Tajiks & Derenko et al., 2010 & 82 & 6 & 7.32 \\
\hline Tatars & Derenko et al., 2010 & 310 & 7 & 2.26 \\
\hline Tatars from Buinsk (Mishars) & Malyarchuk et al., 2010 & 126 & 0 & 0 \\
\hline Telenghits & Derenko et al., 2010 & 71 & 12 & 16.90 \\
\hline Teleuts & Derenko et al., 2010 & 53 & 15 & 28.30 \\
\hline Thai & Derenko et al., 2010 & 552 & 19 & 3.44 \\
\hline Tibetan & Liu et al., 2011 & 40 & 0 & 0 \\
\hline Todjins & Derenko et al., 2010 & 48 & 23 & 47.92 \\
\hline Tofalars & Derenko et al., 2010 & 58 & 36 & 62.07 \\
\hline Tsipya & Grosheva et al., 2014 & 53 & 1 & 1.89 \\
\hline Tubalars & Derenko et al., 2010 & 72 & 14 & 19.44 \\
\hline Tujia & Liu et al., 2011 & 29 & 2 & 6.90 \\
\hline Turkish & Quintana-Murci et al., 2004 & 40 & 0 & 0 \\
\hline Turkmen & Comas et al., 2004 & 20 & 1 & 5.00 \\
\hline Turkmen & Quintana-Murci et al., 2004 & 41 & 3 & 7.32 \\
\hline Turkmenistan & Irwin et al., 2010 & 249 & 17 & 6.83 \\
\hline Turkmens & Derenko et al., 2010 & 178 & 24 & 13.48 \\
\hline Turks & Derenko et al., 2010 & 218 & 4 & 1.83 \\
\hline Tuscans & Zheng et al., 2012 & 105 & 0 & 0 \\
\hline Tuscans & Achilli et al., 2007 & 322 & 0 & 0 \\
\hline Tuvinians & Derenko et al., 2010 & 231 & 109 & 47.19 \\
\hline Tuvinians & Derenko et al., 2007 & 105 & 53 & 50.48 \\
\hline
\end{tabular}




\begin{tabular}{|c|c|c|c|c|}
\hline \multicolumn{5}{|l|}{ Continued } \\
\hline Udegeys & Derenko et al., 2010 & 46 & 8 & 17.39 \\
\hline Udmurts & Derenko et al., 2010 & 189 & 5 & 2.65 \\
\hline Uighurs & Derenko et al., 2010 & 177 & 13 & 7.34 \\
\hline Uighurs, China & Derenko et al., 2010 & 47 & 3 & 6.38 \\
\hline Ukraine & Mielnik-Sikorska et al., 2013 & 159 & 2 & 1.26 \\
\hline Ulchi & Derenko et al., 2010 & 87 & 12 & 13.79 \\
\hline Upper Anadyr (Chuvantsi) & Volodko et al., 2008 & 32 & 10,016 & 31.30 \\
\hline Upper Kolyma (Yukaghirs) & Volodko et al., 2008 & 18 & 12,996 & 72.20 \\
\hline Uygur & Yao et al., 2004 & 47 & 3 & 6.38 \\
\hline Uzbek & Comas et al., 2004 & 20 & 2 & 10.00 \\
\hline Uzbek & Yao et al., 2004 & 58 & 3 & 5.17 \\
\hline Uzbek & Quintana-Murci et al., 2004 & 42 & 1 & 2.38 \\
\hline Uzbekistan & Irwin et al., 2010 & 328 & 27 & 8.23 \\
\hline Uzbeks & Derenko et al., 2010 & 130 & 8 & 6.15 \\
\hline $\mathrm{Va}$ & Liu et al., 2011 & 36 & 0 & 0 \\
\hline West Evenks & Derenko et al., 2010 & 73 & 34 & 46.58 \\
\hline Yakuts & Derenko et al., 2010 & 254 & 119 & 46.85 \\
\hline Yao & Liu et al., 2011 & 40 & 4 & 10.00 \\
\hline Yi & Liu et al., 2011 & 20 & 0 & 0 \\
\hline Yugdon & Grosheva et al., 2014 & 58 & 7 & 12.07 \\
\hline Yukaghirs & Derenko et al., 2010 & 100 & 67 & 67.00 \\
\hline Yukaghirs & Gubina et al., 2013 & 66 & 41 & 62.12 \\
\hline Zhulang & Liu et al., 2011 & 83 & 4 & 4.82 \\
\hline
\end{tabular}

\section{References S2(a)}

Abu-Amero, K. K., Gonzalez, A. M., Larruga, J. M., Bosley, T. M., \& Cabrera, V. M. (2007). Eurasian and African Mitochondrial DNA Influences in the Saudi Arabian Population. BMC Evolutionary Biology, 7, 32. http://dx.doi.org/10.1186/1471-2148-7-32

Abu-Amero, K. K., Larruga, J. M., Cabrera, V. M., \& Gonzalez, A. M. (2008). Mitochondrial DNA Structure in the Arabian Peninsula. BMC Evolutionary Biology, 8, 45. http://dx.doi.org/10.1186/1471-2148-8-45

Achilli, A., Olivieri, A., Pala, M., Metspalu, E., Fornarino, S., Battaglia, V. et al. (2007). Mitochondrial DNA Variation of Modern Tuscans Supports the Near Eastern Origin of Etruscans. American Journal of Human Genetics, 80, 759-768. http://dx.doi.org/10.1086/512822

Álvarez-Iglesias, V., Mosquera-Miguel, A., Cerezo, M., Quintáns, B., Zarrabeitia, M. T., Cuscó, I. et al. (2009). New Population and Phylogenetic Features of the Internal Variation within Mitochondrial DNA Macro-Haplogroup R0. PLoS ONE, 4, e5112. http://dx.doi.org/10.1371/journal.pone.0005112

Al-Zahery, N., Semino, O., Benuzzi, G., Magri, C., Passarino, G., Torroni, A., \& Santachiara-Benerecetti, A. S. (2003). Y-Chromosome and mtDNA Polymorphisms in Iraq, a Crossroad of the Early Human Dispersal and of Post-Neolithic 
Migrations. Molecular Phylogenetics and Evolution, 28, 458-472. http://dx.doi.org/10.1016/S1055-7903(03)00039-3

Brandstatter, A., Klein, R., Duftner, N., Wiegand, P., \& Parson, W. (2006). Application of a Quasi-Median Network Analysis for the Visualization of Character Conflicts to a Population Sample of Mitochondrial DNA Control Region Sequences from Southern Germany (Ulm). International Journal of Legal Medicine, 120, 310-314.

http://dx.doi.org/10.1007/s00414-006-0114-x

Brandstatter, A., Niederstatter, H., Pavlic, M., Grubwieser, P., \& Parson, W. (2007). Generating Population Data for the EMPOP Database-An Overview of the mtDNA Sequencing and Data Evaluation Processes Considering 273 Austrian Control Region Sequences as Example. Forensic Science International, 166, 164-175. http://dx.doi.org/10.1016/j.forsciint.2006.05.006

Comas, D., Plaza, S., Wells, R. S., Yuldaseva, N., Lao, O., Calafell, F., \& Bertranpetit, J. (2004). Admixture, Migrations, and Dispersals in Central Asia: Evidence from Maternal DNA Lineages. European Journal of Human Genetics, 12, 495504. http://dx.doi.org/10.1038/sj.ejhg.5201160

Derenko, M., Malyarchuk, B., Bahmanimehr, A., Denisova, G., Perkova, M., Farjadian, S., \& Yepiskoposyan, L. (2013). Complete Mitochondrial DNA Diversity in Iranians. PLoS ONE, 8, e80673.

http://dx.doi.org/10.1371/journal.pone.0080673

Derenko, M., Malyarchuk, B., Denisova, G., Perkova, M., Rogalla, U., Grzybowski, T. et al. (2012). Complete Mitochondrial DNA Analysis of Eastern Eurasian Haplogroups Rarely Found in Populations of Northern Asia and Eastern Europe. PLoS ONE, 7, e32179. http://dx.doi.org/10.1371/journal.pone.0032179

Derenko, M., Malyarchuk, B., Grzybowski, T., Denisova, G., Dambueva, I., Perkova, M. et al. (2007). Phylogeographic Analysis of Mitochondrial DNA in Northern Asian Populations. The American Journal of Human Genetics, 81, 10251041. http://dx.doi.org/10.1086/522933

Derenko, M., Malyarchuk, B., Grzybowski, T., Denisova, G., Rogalla, U., Perkova, M. et al. (2010). Origin and Post-Glacial Dispersal of Mitochondrial DNA Haplogroups C and D in Northern Asia. PLoS ONE, 5, e15214. http://dx.doi.org/10.1371/journal.pone.0015214

Grosheva, A., Shneider, Y. V., Zhukova, O., Morozova, I. Y., \& Rychkov, S. Y. (2014). Features of the Udmurt Mitochondrial Gene Pool in Relation to Tribal Structure. Russian Journal of Genetics, 50, 975-986.

http://dx.doi.org/10.1134/S1022795414090063

Grzybowski, T., Malyarchuk, B. A., Derenko, M. V., Perkova, M. A., Bednarek, J., \& Wozniak, M. (2007). Complex Interactions of the Eastern and Western Slavic Populations with Other European Groups as Revealed by Mitochondrial DNA Analysis. Forensic Science International: Genetics, 1, 141-147. http://dx.doi.org/10.1016/j.fsigen.2007.01.010

Gubina, M. A., Girgol'kau, L. A., Babenko, V. N., Damba, L. D., Maksimov, V. N., \& Voevoda, M. I. (2013). Mitochondrial DNA Polymorphism in Populations of Aboriginal Residents of the Far East. Russian Journal of Genetics, 49, 751-764. http://dx.doi.org/10.1134/S1022795413070065

Hedman, M., Brandstätter, A., Pimenoff, V., Sistonen, P., Palo, J. U., Parson, W., \& Sajantila, A. (2007). Finnish Mitochondrial DNA HVS-I and HVS-II Population Data. Forensic Science International, 172, 171-178. http://dx.doi.org/10.1016/j.forsciint.2006.09.012

Irwin, J. A., Ikramov, A., Saunier, J., Bodner, M., Amory, S., Rock, A. et al. (2010). The mtDNA Composition of Uzbekistan: A Microcosm of Central Asian Patterns. International Journal of Legal Medicine, 124, 195-204. http://dx.doi.org/10.1007/s00414-009-0406-Z

Irwin, J., Egyed, B., Saunier, J., Szamosi, G., O’Callaghan, J., Padar, Z., \& Parsons, T. (2007). Hungarian mtDNA Population Databases from Budapest and the Baranya County Roma. International Journal of Legal Medicine, 121, 377383. http://dx.doi.org/10.1007/s00414-006-0128-4

Irwin, J., Saunier, J., Strouss, K., Paintner, C., Diegoli, T., Sturk, K. et al. (2008). Mitochondrial Control Region Sequences from Northern Greece and Greek Cypriots. International Journal of Legal Medicine, 122, 87-89.

http://dx.doi.org/10.1007/s00414-007-0173-7

Karachanak, S., Carossa, V., Nesheva, D., Olivieri, A., Pala, M., Hooshiar Kashani, B. et al. (2012). Bulgarians vs the Other European Populations: A Mitochondrial DNA Perspective. International Journal of Legal Medicine, 126, 497-503. http://dx.doi.org/10.1007/s00414-011-0589-y

Kushniarevich, A., Sivitskaya, L., Danilenko, N., Novogrodskii, T., Tsybovsky, I., Kiseleva, A. et al. (2013). Uniparental Genetic Heritage of Belarusians: Encounter of Rare Middle Eastern Matrilineages with a Central European Mitochondrial DNA Pool. PLoS ONE, 8, e66499. http://dx.doi.org/10.1371/journal.pone.0066499

Lehocký, I., Baldovič, M., Kádaši, L., \& Metspalu, E. (2008). A Database of Mitochondrial DNA Hypervariable Regions I and II Sequences of Individuals from Slovakia. Forensic Science International: Genetics, 2, e53-e59.

http://dx.doi.org/10.1016/j.fsigen.2007.12.008 
Liu, C., Wang, S.-Y., Zhao, M., Xu, Z.-Y., Hu, Y.-H., Chen, F. et al. (2011). Mitochondrial DNA Polymorphisms in Gelao Ethnic Group Residing in Southwest China. Forensic Science International: Genetics, 5, e4-e10. http://dx.doi.org/10.1016/j.fsigen.2010.04.007

Malyarchuk, B. A., Grzybowski, T., Derenko, M. V., Czarny, J., Drobnič, K., \& Miścicka-Śliwka, D. (2003). Mitochondrial DNA Variability in Bosnians and Slovenians. Annals of Human Genetics, 67, 412-425. http://dx.doi.org/10.1046/j.1469-1809.2003.00042.x

Malyarchuk, B. A., Vanecek, T., Perkova, M. A., Derenko, M. V., \& Sip, M. (2006). Mitochondrial DNA Variability in the Czech Population, with Application to the Ethnic History of Slavs. Human Biology, 78, 681-696. http://dx.doi.org/10.1353/hub.2007.0014

Malyarchuk, B., Derenko, M., Denisova, G., \& Kravtsova, O. (2010). Mitogenomic Diversity in Tatars from the Volga-Ural Region of Russia. Molecular Biology and Evolution, 27, 2220-2226. http://dx.doi.org/10.1093/molbev/msq065

Mielnik-Sikorska, M., Daca, P., Malyarchuk, B., Derenko, M., Skonieczna, K., Perkova, M. et al. (2013). The History of Slavs Inferred from Complete Mitochondrial Genome Sequences. PLoS ONE, 8, e54360. http://dx.doi.org/10.1371/journal.pone.0054360

Mikkelsen, M., Sorensen, E., Rasmussen, E. M., \& Morling, N. (2010). Mitochondrial DNA HV1 and HV2 Variation in Danes. Forensic Science International: Genetics, 4, e87-88. http://dx.doi.org/10.1016/i.fsigen.2009.07.007

Pimenoff, V. N., Comas, D., Palo, J. U., Vershubsky, G., Kozlov, A., \& Sajantila, A. (2008). Northwest Siberian Khanty and Mansi in the Junction of West and East Eurasian Gene Pools as Revealed by Uniparental Markers. European Journal of Human Genetics, 16, 1254-1264. http://www.nature.com/ejhg/journal/v16/n10/suppinfo/ejhg2008101s1.html http://dx.doi.org/10.1038/ejhg.2008.101

Prieto, L., Zimmermann, B., Goios, A., Rodriguez-Monge, A., Paneto, G. G., Alves, C. et al. (2011). The GHEP-EMPOP Collaboration on mtDNA Population Data-A New Resource for Forensic Casework. Forensic Science International: Genetics, 5, 146-151. http://dx.doi.org/10.1016/i.fsigen.2010.10.013

Quintana-Murci, L., Chaix, R., Wells, R. S., Behar, D. M., Sayar, H., Scozzari, R. et al. (2004). Where West Meets East: The Complex mtDNA Landscape of the Southwest and Central Asian Corridor. American Journal of Human Genetics, 74, 827-845. http://dx.doi.org/10.1086/383236

Richard, C., Pennarun, E., Kivisild, T., Tambets, K., Tolk, H. V., Metspalu, E. et al. (2007). An mtDNA Perspective of French Genetic Variation. Annals of Human Biology, 34, 68-79. http://dx.doi.org/10.1080/03014460601076098

Simonson, T. S., Xing, J., Barrett, R., Jerah, E., Loa, P., Zhang, Y. et al. (2011). Ancestry of the Iban Is Predominantly Southeast Asian: Genetic Evidence from Autosomal, Mitochondrial, and Y Chromosomes. PLoS ONE, 6, e16338. http://dx.doi.org/10.1371/journal.pone.0016338

Turchi, C., Buscemi, L., Previdere, C., Grignani, P., Brandstatter, A., Achilli, A. et al. (2008). Italian Mitochondrial DNA Database: Results of a Collaborative Exercise and Proficiency Testing. International Journal of Legal Medicine, 122, 199-204. http://dx.doi.org/10.1007/s00414-007-0207-1

Volodko, N. V., Starikovskaya, E. B., Mazunin, I. O., Eltsov, N. P., Naidenko, P. V., Wallace, D. C., \& Sukernik, R. I. (2008). Mitochondrial Genome Diversity in Arctic Siberians, with Particular Reference to the Evolutionary History of Beringia and Pleistocenic Peopling of the Americas. The American Journal of Human Genetics, 82, 1084-1100. http://dx.doi.org/10.1016/j.ajhg.2008.03.019

Yao, Y. G., Kong, Q. P., Wang, C. Y., Zhu, C. L., \& Zhang, Y. P. (2004). Different Matrilineal Contributions to Genetic Structure of Ethnic Groups in the Silk Road Region in China. Molecular Biology and Evolution, 21, 2265-2280. http://dx.doi.org/10.1093/molbev/msh238

Yao, Y.-G., Kong, Q.-P., Bandelt, H.-J., Kivisild, T. et al. (2002). Phylogeographic Differentiation of Mitochondrial DNA in Han Chinese. The American Journal of Human Genetics, 70, 635-651. http://dx.doi.org/10.1086/338999

Zgonjanin, D., Veselinovic, I., Kubat, M., Furac, I., Antov, M., Loncar, E., \& Omorjan, R. (2010). Sequence Polymorphism of the Mitochondrial DNA Control Region in the Population of Vojvodina Province, Serbia. Legal medicine (Tokyo, Japan), 12, 104-107. http://dx.doi.org/10.1016/j.legalmed.2009.10.007

Zhao, Y. B., Sun, W. Y., Zhan, Y., Di, W., \& Yu, C. C. (2011). Mitochondrial DNA Evidence of Southward Migration of Manchus in China. Molekuliarnaia Biologiia (Moskva), 45, 825-830. http://dx.doi.org/10.1134/s0026893311050153

Zheng, H.-X., Yan, S., Qin, Z.-D., \& Jin, L. (2012). MtDNA Analysis of Global Populations Support That Major Population Expansions Began before Neolithic Time. Scientific Reports, 2, 745. http://dx.doi.org/10.1038/srep00745

Zimmermann, B., Brandstatter, A., Duftner, N., Niederwieser, D., Spiroski, M., Arsov, T., \& Parson, W. (2007). Mitochondrial DNA Control Region Population Data from Macedonia. Forensic Science International: Genetics, 1, e4-e9.

http://dx.doi.org/10.1016/j.fsigen.2007.03.002 
(b)

\begin{tabular}{|c|c|c|c|c|}
\hline Country & Reference & $\mathrm{N}$ & Hg D & Hg D (\%) \\
\hline Adam & Grosheva et al., 2014 & 54 & 1 & 1.85 \\
\hline Afghanistan & Irwin et al., 2010 & 98 & 2 & 2.04 \\
\hline Ainu & Derenko et al., 2010 & 51 & 9 & 17.65 \\
\hline Altaian Kazakhs & Derenko et al., 2012 & 98 & 26 & 26.53 \\
\hline Altaians-Kizhi & Derenko et al., 2007 & 90 & 8 & 8.89 \\
\hline Altaians & Derenko et al., 2010 & 110 & 17 & 15.45 \\
\hline Ambon & Simonson et al., 2011 & 43 & 2 & 4.65 \\
\hline Asian Eskimos & Gubina et al., 2013 & 40 & 6 & 15.00 \\
\hline Austrians & Brandsta"tter et al., 2007 & 273 & 0 & 0 \\
\hline Azeris & Derenko et al., 2013 & 22 & 0 & 0 \\
\hline Bai & Liu et al., 2011 & 68 & 13 & 19.12 \\
\hline Bali & Simonson et al., 2011 & 82 & 2 & 2.44 \\
\hline Baluch & Quintana-Murci et al., 2004 & 39 & 0 & 0 \\
\hline Barghuts & Derenko et al., 2012 & 149 & 53 & 35.57 \\
\hline Bashkirs & Derenko et al., 2010 & 207 & 20 & 9.66 \\
\hline Basque Country & Prieto et al., 2011 & 29 & 0 & 0 \\
\hline Basques from Northern Navarre & Cardoso et al., 2011 & 110 & 0 & 0 \\
\hline Belarusians & Kushniarevich et al., 2013 & 267 & 1 & 0.37 \\
\hline Borneo & Simonson et al., 2011 & 157 & 5 & 3.18 \\
\hline Bosnians & Malyarchuk et al., 2003 & 144 & 0 & 0 \\
\hline Brahui & Quintana-Murci et al., 2004 & 38 & 1 & 2.63 \\
\hline British people & Zheng et al., 2012 & 94 & 0 & 0 \\
\hline Bukharan Arabs & Comas et al., 2004 & 20 & 0 & 0 \\
\hline Bulgarians & Karachanak et al., 2012 & 855 & 3 & 0.35 \\
\hline Buryats & Derenko et al., 2010 & 419 & 146 & 34.84 \\
\hline Buryats & Derenko et al., 2007 & 295 & 103 & 34.92 \\
\hline Cantabria & Álvarez-Iglesias et al., 2009 & 135 & 0 & 0 \\
\hline Catalonia & Álvarez-Iglesias et al., 2009 & 101 & 2 & 1.98 \\
\hline Central Portugal & Prieto et al., 2011 & 53 & 0 & 0 \\
\hline Chaplin & Volodko et al., 2008 & 50 & 5 & 10.00 \\
\hline Chinese & Derenko et al., 2010 & 1930 & 317 & 16.42 \\
\hline Chukchi & Derenko et al., 2010 & 417 & 64 & 15.35 \\
\hline Chukchi & Gubina et al., 2013 & 159 & 31 & 19.50 \\
\hline
\end{tabular}




\begin{tabular}{|c|c|c|c|c|}
\hline \multicolumn{5}{|l|}{ Continued } \\
\hline Chukchi & Derenko et al., 2007 & 15 & 2 & 13.33 \\
\hline Chukchi & Volodko et al., 2008 & 182 & 13 & 7.14 \\
\hline Chutyr' & Grosheva et al., 2014 & 63 & 5 & 7.94 \\
\hline Chuvantsi & Derenko et al., 2010 & 32 & 3 & 9.38 \\
\hline Chuvashis & Derenko et al., 2010 & 142 & 4 & 2.82 \\
\hline Crimean Tatars & Comas et al., 2004 & 20 & 0 & 0 \\
\hline Croats & Derenko et al., 2010 & 407 & 2 & 0.49 \\
\hline Cypriots & Irwin et al., 2008 & 91 & 0 & 0 \\
\hline Czech & Mielnik-Sikorska et al., 2013 & 85 & 0 & 0 \\
\hline Czechs & Derenko et al., 2010 & 354 & 1 & 0.28 \\
\hline Czechs (Western Bohemia) & Malyarchuk et al., 2006 & 179 & 1 & 0.56 \\
\hline Dai & Liu et al., 2011 & 41 & 4 & 9.76 \\
\hline Danes & Mikkelsen et al., 2010 & 201 & 0 & 0 \\
\hline Daurs, China & Derenko et al., 2010 & 45 & 11 & 24.44 \\
\hline Dolgans & Derenko et al., 2010 & 157 & 62 & 39.49 \\
\hline Dong & Liu et al., 2011 & 28 & 0 & 0 \\
\hline Dungan & Comas et al., 2004 & 16 & 1 & 6.25 \\
\hline East Evenks & Derenko et al., 2010 & 45 & 11 & 24.44 \\
\hline East Evenks & Derenko et al., 2007 & 45 & 11 & 24.44 \\
\hline European Russia (Novgorod oblast) & Grzybowski et al., 2007 & 157 & 2 & 1.27 \\
\hline Evenks & Derenko et al., 2010 & 71 & 15 & 21.13 \\
\hline Evenks & Gubina et al., 2013 & 38 & 11 & 28.95 \\
\hline Evenks, China & Derenko et al., 2010 & 47 & 15 & 31.91 \\
\hline Evens & Derenko et al., 2010 & 191 & 41 & 21.47 \\
\hline Eskimos & Derenko et al., 2010 & 254 & 49 & 19.29 \\
\hline Ezhevo & Grosheva et al., 2014 & 39 & 3 & 7.69 \\
\hline Finns & Hedman et al., 2007 & 200 & 0 & 0 \\
\hline Fins & Zheng et al., 2012 & 97 & 0 & 0 \\
\hline French people & Richard et al., 2007 & 868 & 0 & 0 \\
\hline Gelao & Liu et al., 2011 & 177 & 27 & 15.25 \\
\hline Georgians & Derenko et al., 2010 & 57 & 3 & 5.26 \\
\hline Germans & Derenko et al., 2010 & 554 & 1 & 0.18 \\
\hline Gilaki & Quintana-Murci et al., 2004 & 37 & 1 & 2.70 \\
\hline Gujarati & Quintana-Murci et al., 2004 & 34 & 0 & 0 \\
\hline
\end{tabular}




\section{Continued}

Han Chinese

Hazara

Hui

Hui, China

Hungarians

Hunza Burusho

Hungarians (Budapest)

Iranians

Iranians

Iraqis

India

Itelmens

Japanese

Java

Kabachigurt

Kalash

Kalmyks

Kalmyks

Karakalpaks

Karelians

Kazak

Kazak

Kazakhs

Kazakhs, China

Kazakhstan

Kazan Tatars (Azmakaevo)

Kets

Khakassians

Khakassians

Khamnigans

Khamnigans

Khants

Khanty

Khoremian Uzbek
Yao et al., 2002

Quintana-Murci et al., 2004

Yao et al., 2004

Derenko et al., 2010

Derenko et al., 2010

Quintana-Murci et al., 2004

Irwin et al., 2007

Derenko et al., 2013

Comas et al., 2004

Al-Zahery et al., 2003

Derenko et al., 2010

Derenko et al., 2010

Derenko et al., 2010

Simonson et al., 2011

Grosheva et al., 2014

Quintana-Murci et al., 2004

Derenko et al., 2010

Derenko et al., 2007

Derenko et al., 2010

Derenko et al., 2010

Comas et al., 2004

Yao et al., 2004

Derenko et al., 2010

Derenko et al., 2010

Irwin et al., 2010

Malyarchuk et al., 2010

Derenko et al., 2010

Derenko et al., 2010

Derenko et al., 2007

Derenko et al., 2010

Derenko et al., 2007

Derenko et al., 2010

Pimenoff et al., 2008

Comas et al., 2004
263

23

45

45

344

44

211

352

20

216

2544

46

1312

46

57

44

110

110

108

512

20

53

511

53

256

71

104

110

57

99

99

106

106

20
21.67

0

15.56

15.56

0.58

0

0.95

1.14

5.00

0

1.81

0

37.50

2.17

8.77

0

29.09

29.09

17.59

3.71

10.00

13.21

19.96

13.21

17.19

9.86

2.88

14.55

15.79

31.31

33.33

16.04

16.04

10.00 


\section{Continued}

Kirghiz

Komis

Koreans

Koreans, China

Koreans

Koryaks

Koryaks

Kurdish

Kurdish-Western Iran

Kurdish-Turkmenistan

Kurds

Kuzyumovo

Kyrgyz

Kyrgyzstan

$$
\text { Lahu }
$$

Lesagurt

Lombok

Lower Kolyma-Indigirka

$$
\text { Lur }
$$

Macedonians (FYROM)

Makrani

Manchu

Mansi

Mansi

Maris

Mazandarian

Miao

Mongolian

Mongolians

Mongolians

Mongolians, China

Mordvins

Mulam

Nanaians
Derenko et al., 2010

Derenko et al., 2010

Derenko et al., 2010

Derenko et al., 2010

Derenko et al., 2007

Derenko et al., 2010

Gubina et al., 2013

Derenko et al., 2010

Quintana-Murci et al., 2004

Quintana-Murci et al., 2004

Derenko et al., 2007

Grosheva et al., 2014

Comas et al., 2004

Irwin et al., 2010

Liu et al., 2011

Grosheva et al., 2014

Simonson et al., 2011

Volodko et al., 2008

Quintana-Murci et al., 2004

Zimmermann et al., 2007

Quintana-Murci et al., 2004

Zhao et al., 2011

Derenko et al., 2010

Pimenoff et al., 2008

Derenko et al., 2010

Quintana-Murci et al., 2004

Liu et al., 2011

Yao et al., 2004

Derenko et al., 2010

Derenko et al., 2007

Derenko et al., 2010

Derenko et al., 2010

Liu et al., 2011

Gubina et al., 2013
200

121

1297

48

103

182

69

135

20

32

25

72

20

249

35

63

44

82

17

200

33

47

161

63

234

21

103

49

150

47

97

120

39

86
17.00

1.65

33.31

33.33

39.81

1.10

2.90

4.44

0

0

12.00

12.50

20.00

20.88

14.29

7.94

6.82

17.07

0

0

0

42.55

12.42

19.05

1.28

0

17.48

20.41

22.67

10.64

29.90

0.83

15.38

27.91 


\section{Continued}

\begin{tabular}{|c|c|c|c|c|}
\hline Nanais & Derenko et al., 2010 & 85 & 22 & 25.88 \\
\hline Naukan & Volodko et al., 2008 & 39 & 10 & 25.64 \\
\hline Naxi & Liu et al., 2011 & 45 & 2 & 4.44 \\
\hline Negidals & Derenko et al., 2010 & 33 & 8 & 24.24 \\
\hline Nenets & Derenko et al., 2010 & 58 & 13 & 22.41 \\
\hline Nenets Tundra & Derenko et al., 2010 & 70 & 4 & 5.71 \\
\hline Nganasan & Volodko et al., 2008 & 39 & 10 & 25.64 \\
\hline Nganasans & Derenko et al., 2010 & 78 & 22 & 28.21 \\
\hline Nivkhs & Derenko et al., 2010 & 56 & 16 & 28.57 \\
\hline Nivkhs & Gubina et al., 2013 & 17 & 3 & 17.65 \\
\hline Nogays & Derenko et al., 2010 & 206 & 16 & 7.77 \\
\hline North and Central Italians & Turchi et al., 2008 & 395 & 3 & 0.76 \\
\hline North Portugal & Prieto et al., 2011 & 187 & 1 & 0.53 \\
\hline Northern Caucasians & Derenko et al., 2010 & 199 & 8 & 4.02 \\
\hline Northern Greeks & Irwin et al., 2008 & 319 & 0 & 0 \\
\hline $\mathrm{Nu}$ & Liu et al., 2011 & 30 & 6 & 20.00 \\
\hline Orochens, China & Derenko et al., 2010 & 44 & 19 & 43.18 \\
\hline Oroks & Derenko et al., 2010 & 61 & 42 & 68.85 \\
\hline Pakistani & Derenko et al., 2010 & 189 & 2 & 1.06 \\
\hline Parsis & Quintana-Murci et al., 2004 & 44 & 0 & 0 \\
\hline Pathan & Quintana-Murci et al., 2004 & 44 & 0 & 0 \\
\hline Persian & Quintana-Murci et al., 2004 & 42 & 0 & 0 \\
\hline Persians & Derenko et al., 2010 & 518 & 7 & 1.35 \\
\hline Persians & Derenko et al., 2013 & 181 & 1 & 0.55 \\
\hline Phillipines & Simonson et al., 2011 & 61 & 5 & 8.20 \\
\hline Poland (Kashubians) & Mielnik-Sikorska et al., 2013 & 203 & 0 & 0 \\
\hline Poland (Podhale) & Mielnik-Sikorska et al., 2013 & 201 & 1 & 0.50 \\
\hline Poland Northeastern & Grzybowski et al., 2007 & 73 & 0 & 0 \\
\hline Poland Northern & Grzybowski et al., 2007 & 253 & 0 & 0 \\
\hline Poland Southern & Grzybowski et al., 2007 & 87 & 0 & 0 \\
\hline Poles & Derenko et al., 2010 & 947 & 3 & 0.32 \\
\hline Qashqais & Derenko et al., 2013 & 112 & 2 & 1.79 \\
\hline Romanians & Derenko et al., 2010 & 360 & 0 & 0 \\
\hline Saudi Arabs & Abu-Amero et al., 2008, 2007 & 553 & 0 & 0 \\
\hline Scandinavians & Derenko et al., 2010 & 316 & 1 & 0.32 \\
\hline Selkups & Derenko et al., 2010 & 120 & 4 & 3.33 \\
\hline Serbia-Vojvodina Province & Zgonjanin et al., 2010 & 104 & 0 & 0 \\
\hline Shors & Derenko et al., 2010 & 82 & 10 & 12.20 \\
\hline Shors & Derenko et al., 2007 & 82 & 10 & 12.20 \\
\hline Shugnan & Quintana-Murci et al., 2004 & 44 & 0 & 0 \\
\hline
\end{tabular}




\begin{tabular}{|c|c|c|c|c|}
\hline Continued & & & & \\
\hline Shui & Liu et al., 2011 & 30 & 1 & 3.33 \\
\hline Sindhi & Quintana-Murci et al., 2004 & 23 & 0 & 0 \\
\hline Sireniki & Volodko et al., 2008 & 37 & 11 & 29.73 \\
\hline Slovaks & Lehocký et al., 2008 & 374 & 0 & 0 \\
\hline Slovenians & Malyarchuk et al., 2003 & 104 & 0 & 0 \\
\hline Sojots & Derenko et al., 2010 & 30 & 14 & 46.67 \\
\hline Southern Germany (Ulm) & Brandsta"tter et al., 2006 & 100 & 0 & 0 \\
\hline Spain & Prieto et al., 2011 & 403 & 0 & 0 \\
\hline Staraya Salya & Grosheva et al., 2014 & 54 & 12 & 22.22 \\
\hline Sulawesi & Simonson et al., 2011 & 237 & 19 & 8.02 \\
\hline Sumatra & Simonson et al., 2011 & 180 & 0 & 0 \\
\hline Sumba & Simonson et al., 2011 & 50 & 1 & 2.00 \\
\hline Svetozarevo & Grosheva et al., 2014 & 45 & 6 & 13.33 \\
\hline Taiwan & Simonson et al., 2011 & 78 & 1 & 1.28 \\
\hline Tajik & Comas et al., 2004 & 20 & 3 & 15.00 \\
\hline Tajikistan & Irwin et al., 2010 & 244 & 24 & 9.84 \\
\hline Tajiks & Derenko et al., 2010 & 82 & 4 & 4.88 \\
\hline Tajiks & Derenko et al., 2007 & 44 & 3 & 6.82 \\
\hline Tatars & Derenko et al., 2010 & 310 & 9 & 2.90 \\
\hline Tatars from Buinsk (Mishars) & Malyarchuk et al., 2010 & 126 & 6 & 4.76 \\
\hline Telenghits & Derenko et al., 2010 & 71 & 15 & 21.13 \\
\hline Telenghits & Derenko et al., 2007 & 71 & 15 & 21.13 \\
\hline Teleuts & Derenko et al., 2010 & 53 & 13 & 24.53 \\
\hline Teleuts & Derenko et al., 2007 & 53 & 13 & 24.53 \\
\hline Thai & Derenko et al., 2010 & 552 & 93 & 16.85 \\
\hline Tibetan & Liu et al., 2011 & 40 & 2 & 5.00 \\
\hline Todjins & Derenko et al., 2010 & 48 & 2 & 4.17 \\
\hline Tofalars & Derenko et al., 2010 & 58 & 0 & 0 \\
\hline Tsipya & Grosheva et al., 2014 & 53 & 7 & 13.21 \\
\hline Tubalars & Derenko et al., 2010 & 72 & 14 & 19.44 \\
\hline Tujia & Liu et al., 2011 & 29 & 0 & 0 \\
\hline Turkish & Quintana-Murci et al., 2004 & 40 & 0 & 0 \\
\hline Turkmen & Comas et al., 2004 & 20 & 6 & 30.00 \\
\hline Turkmen & Quintana-Murci et al., 2004 & 41 & 9 & 21.95 \\
\hline Turkmenistan & Irwin et al., 2010 & 249 & 26 & 10.44 \\
\hline Turkmens & Derenko et al., 2010 & 178 & 29 & 16.29 \\
\hline Turks & Derenko et al., 2010 & 218 & 5 & 2.29 \\
\hline Tuscans & Zheng et al., 2012 & 105 & 1 & 0.95 \\
\hline Tuscans & Achilli et al., 2007 & 322 & 1 & 0.31 \\
\hline
\end{tabular}




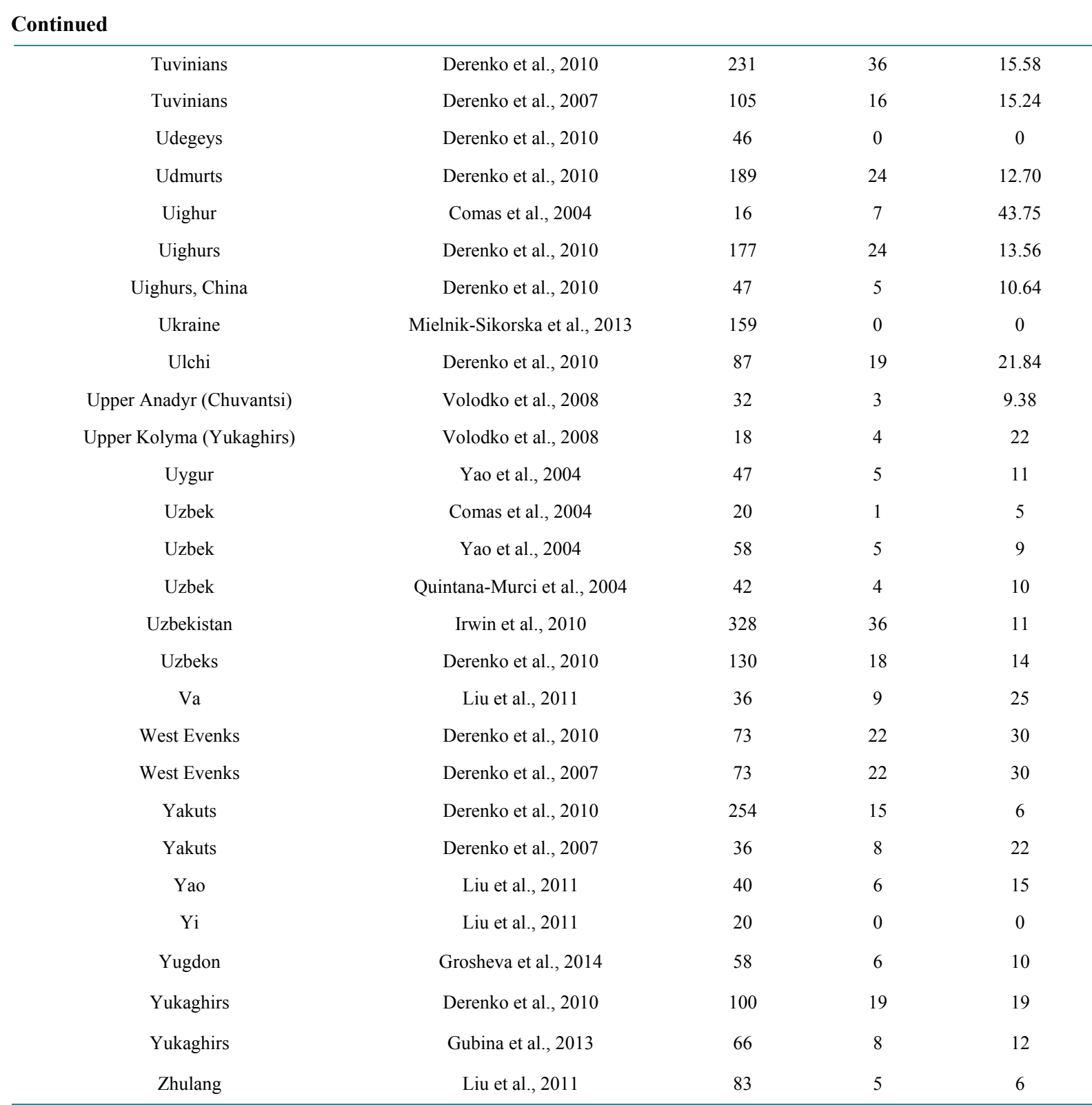

\section{References S2(b)}

Abu-Amero, K. K., Gonzalez, A. M., Larruga, J. M., Bosley, T. M., \& Cabrera, V. M. (2007). Eurasian and African Mitochondrial DNA Influences in the Saudi Arabian Population. BMC Evolutionary Biology, 7, 32. http://dx.doi.org/10.1186/1471-2148-7-32

Abu-Amero, K. K., Larruga, J. M., Cabrera, V. M., \& Gonzalez, A. M. (2008). Mitochondrial DNA Structure in the Arabian Peninsula. BMC Evolutionary Biology, 8, 45. http://dx.doi.org/10.1186/1471-2148-8-45

Achilli, A., Olivieri, A., Pala, M., Metspalu, E., Fornarino, S., Battaglia, V. et al. (2007). Mitochondrial DNA Variation of Modern Tuscans Supports the Near Eastern Origin of Etruscans. American Journal of Human Genetics, 80, 759-768. http://dx.doi.org/10.1086/512822

Álvarez-Iglesias, V., Mosquera-Miguel, A., Cerezo, M., Quintáns, B., Zarrabeitia, M. T., Cuscó, I. et al. (2009). New Population and Phylogenetic Features of the Internal Variation within Mitochondrial DNA Macro-Haplogroup R0. PLoS ONE, 4, e5112. http://dx.doi.org/10.1371/journal.pone.0005112

Al-Zahery, N., Semino, O., Benuzzi, G., Magri, C., Passarino, G., Torroni, A., \& Santachiara-Benerecetti, A. S. (2003). YChromosome and mtDNA Polymorphisms in Iraq, a Crossroad of the Early Human Dispersal and of Post-Neolithic 
Migrations. Molecular Phylogenetics and Evolution, 28, 458-472. http://dx.doi.org/10.1016/S1055-7903(03)00039-3

Brandstatter, A., Klein, R., Duftner, N., Wiegand, P., \& Parson, W. (2006). Application of a Quasi-Median Network Analysis for the Visualization of Character Conflicts to a Population Sample of Mitochondrial DNA Control Region Sequences from Southern Germany (Ulm). International Journal of Legal Medicine, 120, 310-314.

http://dx.doi.org/10.1007/s00414-006-0114-x

Brandstatter, A., Niederstatter, H., Pavlic, M., Grubwieser, P., \& Parson, W. (2007). Generating Population Data for the EMPOP Database-An Overview of the mtDNA Sequencing and Data Evaluation Processes Considering 273 Austrian Control Region Sequences as Example. Forensic Science International, 166, 164-175.

http://dx.doi.org/10.1016/j.forsciint.2006.05.006

Cardoso, S., Alfonso-Sanchez, M. A., Valverde, L., Odriozola, A., Perez-Miranda, A. M., Pena, J. A., \& de Pancorbo, M. M. (2011). The Maternal Legacy of Basques in Northern Navarre: New Insights into the Mitochondrial DNA Diversity of the Franco-Cantabrian Area. American Journal of Physical Anthropology, 145, 480-488. http://dx.doi.org/10.1002/ajpa.21532

Comas, D., Plaza, S., Wells, R. S., Yuldaseva, N., Lao, O., Calafell, F., \& Bertranpetit, J. (2004). Admixture, Migrations, and Dispersals in Central Asia: Evidence from Maternal DNA Lineages. European Journal of Human Genetics, 12, 495504. http://dx.doi.org/10.1038/sj.ejhg.5201160

Derenko, M., Malyarchuk, B., Bahmanimehr, A., Denisova, G., Perkova, M., Farjadian, S., \& Yepiskoposyan, L. (2013). Complete Mitochondrial DNA Diversity in Iranians. PLoS ONE, 8, e80673.

http://dx.doi.org/10.1371/journal.pone.0080673

Derenko, M., Malyarchuk, B., Denisova, G., Perkova, M., Rogalla, U., Grzybowski, T. et al. (2012). Complete Mitochondrial DNA Analysis of Eastern Eurasian Haplogroups Rarely Found in Populations of Northern Asia and Eastern Europe. PLoS ONE, 7, e32179. http://dx.doi.org/10.1371/journal.pone.0032179

Derenko, M., Malyarchuk, B., Grzybowski, T., Denisova, G., Dambueva, I., Perkova, M. et al. (2007). Phylogeographic Analysis of Mitochondrial DNA in Northern Asian Populations. The American Journal of Human Genetics, 81, 10251041. http://dx.doi.org/10.1086/522933

Derenko, M., Malyarchuk, B., Grzybowski, T., Denisova, G., Rogalla, U., Perkova, M. et al. (2010). Origin and Post-Glacial Dispersal of Mitochondrial DNA Haplogroups C and D in Northern Asia. PLoS ONE, 5, e15214. http://dx.doi.org/10.1371/journal.pone.0015214

Grosheva, A., Shneider, Y. V., Zhukova, O., Morozova, I. Y., \& Rychkov, S. Y. (2014). Features of the Udmurt Mitochondrial Gene Pool in Relation to Tribal Structure. Russian Journal of Genetics, 50, 975-986. http://dx.doi.org/10.1134/S1022795414090063

Grzybowski, T., Malyarchuk, B. A., Derenko, M. V., Perkova, M. A., Bednarek, J., \& Wozniak, M. (2007). Complex Interactions of the Eastern and Western Slavic Populations with Other European Groups as Revealed by Mitochondrial DNA Analysis. Forensic Science International: Genetics, 1, 141-147. http://dx.doi.org/10.1016/j.fsigen.2007.01.010

Gubina, M. A., Girgol'kau, L. A., Babenko, V. N., Damba, L. D., Maksimov, V. N., \& Voevoda, M. I. (2013). Mitochondrial DNA Polymorphism in Populations of Aboriginal Residents of the Far East. Russian Journal of Genetics, 49, 751-764. http://dx.doi.org/10.1134/S1022795413070065

Hedman, M., Brandstätter, A., Pimenoff, V., Sistonen, P., Palo, J. U., Parson, W., \& Sajantila, A. (2007). Finnish Mitochondrial DNA HVS-I and HVS-II Population Data. Forensic Science International, 172, 171-178. http://dx.doi.org/10.1016/j.forsciint.2006.09.012

Irwin, J. A., Ikramov, A., Saunier, J., Bodner, M., Amory, S., Rock, A. et al. (2010). The mtDNA Composition of Uzbekistan: A Microcosm of Central Asian Patterns. International Journal of Legal Medicine, 124, 195-204. http://dx.doi.org/10.1007/s00414-009-0406-Z

Irwin, J., Egyed, B., Saunier, J., Szamosi, G., O’Callaghan, J., Padar, Z., \& Parsons, T. (2007). Hungarian mtDNA Population Databases from Budapest and the Baranya County Roma. International Journal of Legal Medicine, 121, 377383. http://dx.doi.org/10.1007/s00414-006-0128-4

Irwin, J., Saunier, J., Strouss, K., Paintner, C., Diegoli, T., Sturk, K. et al. (2008). Mitochondrial Control Region Sequences from Northern Greece and Greek Cypriots. International Journal of Legal Medicine, 122, 87-89. http://dx.doi.org/10.1007/s00414-007-0173-7

Karachanak, S., Carossa, V., Nesheva, D., Olivieri, A., Pala, M., Hooshiar Kashani, B. et al. (2012). Bulgarians vs the Other European Populations: A Mitochondrial DNA Perspective. International Journal of Legal Medicine, 126, 497-503. http://dx.doi.org/10.1007/s00414-011-0589-y

Kushniarevich, A., Sivitskaya, L., Danilenko, N., Novogrodskii, T., Tsybovsky, I., Kiseleva, A. et al. (2013). Uniparental Genetic Heritage of Belarusians: Encounter of Rare Middle Eastern Matrilineages with a Central European Mitochondrial DNA Pool. PLoS ONE, 8, e66499. http://dx.doi.org/10.1371/journal.pone.0066499

Lehocký, I., Baldovič, M., Kádaši, L', \& Metspalu, E. (2008). A Database of Mitochondrial DNA Hypervariable Regions I and II Sequences of Individuals from Slovakia. Forensic Science International: Genetics, 2, e53-e59. 
http://dx.doi.org/10.1016/j.fsigen.2007.12.008

Liu, C., Wang, S.-Y., Zhao, M., Xu, Z.-Y., Hu, Y.-H., Chen, F. et al. (2011). Mitochondrial DNA Polymorphisms in Gelao Ethnic Group Residing in Southwest China. Forensic Science International: Genetics, 5, e4-e10. http://dx.doi.org/10.1016/j.fsigen.2010.04.007

Malyarchuk, B. A., Grzybowski, T., Derenko, M. V., Czarny, J., Drobnič, K., \& Miścicka-Śliwka, D. (2003). Mitochondrial DNA Variability in Bosnians and Slovenians. Annals of Human Genetics, 67, 412-425.

http://dx.doi.org/10.1046/j.1469-1809.2003.00042.x

Malyarchuk, B. A., Vanecek, T., Perkova, M. A., Derenko, M. V., \& Sip, M. (2006). Mitochondrial DNA Variability in the Czech Population, with Application to the Ethnic History of Slavs. Human Biology, 78, 681-696. http://dx.doi.org/10.1353/hub.2007.0014

Malyarchuk, B., Derenko, M., Denisova, G., \& Kravtsova, O. (2010). Mitogenomic Diversity in Tatars from the Volga-Ural Region of Russia. Molecular Biology and Evolution, 27, 2220-2226. http://dx.doi.org/10.1093/molbev/msq065

Mielnik-Sikorska, M., Daca, P., Malyarchuk, B., Derenko, M., Skonieczna, K., Perkova, M. et al. (2013). The History of Slavs Inferred from Complete Mitochondrial Genome Sequences. PLoS ONE, 8, e54360. http://dx.doi.org/10.1371/journal.pone.0054360

Mikkelsen, M., Sorensen, E., Rasmussen, E. M., \& Morling, N. (2010). Mitochondrial DNA HV1 and HV2 Variation in Danes. Forensic Science International: Genetics, 4, e87-88. http://dx.doi.org/10.1016/i.fsigen.2009.07.007

Pimenoff, V. N., Comas, D., Palo, J. U., Vershubsky, G., Kozlov, A., \& Sajantila, A. (2008). Northwest Siberian Khanty and Mansi in the Junction of West and East Eurasian Gene Pools as Revealed by Uniparental Markers. European Journal of Human Genetics, 16, 1254-1264. http://www.nature.com/ejhg/journal/v16/n10/suppinfo/ejhg2008101s1.html http://dx.doi.org/10.1038/ejhg.2008.101

Prieto, L., Zimmermann, B., Goios, A., Rodriguez-Monge, A., Paneto, G. G., Alves, C. et al. (2011). The GHEP-EMPOP Collaboration on mtDNA Population Data-A New Resource for Forensic Casework. Forensic Science International: Genetics, 5, 146-151. http://dx.doi.org/10.1016/j.fsigen.2010.10.013

Quintana-Murci, L., Chaix, R., Wells, R. S., Behar, D. M., Sayar, H., Scozzari, R. et al. (2004). Where West Meets East: The Complex mtDNA Landscape of the Southwest and Central Asian Corridor. American Journal of Human Genetics, 74, 827-845. http://dx.doi.org/10.1086/383236

Richard, C., Pennarun, E., Kivisild, T., Tambets, K., Tolk, H. V., Metspalu, E. et al. (2007). An mtDNA Perspective of French Genetic Variation. Annals of Human Biology, 34, 68-79. http://dx.doi.org/10.1080/03014460601076098

Simonson, T. S., Xing, J., Barrett, R., Jerah, E., Loa, P., Zhang, Y. et al. (2011). Ancestry of the Iban Is Predominantly Southeast Asian: Genetic Evidence from Autosomal, Mitochondrial, and Y Chromosomes. PLoS ONE, 6, e16338. http://dx.doi.org/10.1371/journal.pone.0016338

Turchi, C., Buscemi, L., Previdere, C., Grignani, P., Brandstatter, A., Achilli, A. et al. (2008). Italian Mitochondrial DNA Database: Results of a Collaborative Exercise and Proficiency Testing. International Journal of Legal Medicine, 122, 199-204. http://dx.doi.org/10.1007/s00414-007-0207-1

Volodko, N. V., Starikovskaya, E. B., Mazunin, I. O., Eltsov, N. P., Naidenko, P. V., Wallace, D. C., \& Sukernik, R. I. (2008). Mitochondrial Genome Diversity in Arctic Siberians, with Particular Reference to the Evolutionary History of Beringia and Pleistocenic Peopling of the Americas. The American Journal of Human Genetics, 82, 1084-1100. http://dx.doi.org/10.1016/j.ajhg.2008.03.019

Yao, Y. G., Kong, Q. P., Wang, C. Y., Zhu, C. L., \& Zhang, Y. P. (2004). Different Matrilineal Contributions to Genetic Structure of Ethnic Groups in the Silk Road Region in China. Molecular Biology and Evolution, 21, 2265-2280. http://dx.doi.org/10.1093/molbev/msh238

Yao, Y.-G., Kong, Q.-P., Bandelt, H.-J., Kivisild, T. et al. (2002). Phylogeographic Differentiation of Mitochondrial DNA in Han Chinese. The American Journal of Human Genetics, 70, 635-651. http://dx.doi.org/10.1086/338999

Zgonjanin, D., Veselinovic, I., Kubat, M., Furac, I., Antov, M., Loncar, E., \& Omorjan, R. (2010). Sequence Polymorphism of the Mitochondrial DNA Control Region in the Population of Vojvodina Province, Serbia. Legal medicine (Tokyo, Japan), 12, 104-107. http://dx.doi.org/10.1016/j.legalmed.2009.10.007

Zhao, Y. B., Sun, W. Y., Zhan, Y., Di, W., \& Yu, C. C. (2011). Mitochondrial DNA Evidence of Southward Migration of Manchus in China. Molekuliarnaia Biologiia (Moskva), 45, 825-830. http://dx.doi.org/10.1134/s0026893311050153

Zheng, H.-X., Yan, S., Qin, Z.-D., \& Jin, L. (2012). MtDNA Analysis of Global Populations Support That Major Population Expansions Began before Neolithic Time. Scientific Reports, 2, 745. http://dx.doi.org/10.1038/srep00745

Zimmermann, B., Brandstatter, A., Duftner, N., Niederwieser, D., Spiroski, M., Arsov, T., \& Parson, W. (2007). Mitochondrial DNA Control Region Population Data from Macedonia. Forensic Science International: Genetics, 1, e4-e9.

http://dx.doi.org/10.1016/j.fsigen.2007.03.002 
(c)

\begin{tabular}{|c|c|c|c|c|}
\hline Country & Reference & $\mathrm{N}$ & $\mathrm{Hg} \mathrm{Z}$ & Hg Z (\%) \\
\hline Adam & Grosheva et al., 2014 & 54 & 3 & 5.56 \\
\hline Afghanistan & Irwin et al., 2010 & 98 & 2 & 2.04 \\
\hline Altaian Kazakhs & Derenko et al., 2012 & 98 & 2 & 2.04 \\
\hline Altaians-Kizhi & Derenko et al., 2007 & 90 & 2 & 2.22 \\
\hline Ambon & Simonson et al., 2011 & 43 & 0 & 0 \\
\hline Asian Eskimos & Gubina et al., 2013 & 40 & 0 & 0 \\
\hline Austrians & Brandsta"tter et al., 2007 & 273 & 0 & 0 \\
\hline Azeris & Derenko et al., 2013 & 22 & 0 & 0 \\
\hline Bai & Liu et al., 2011 & 68 & 0 & 0 \\
\hline Bali & Simonson et al., 2011 & 82 & 0 & 0 \\
\hline Baluch & Quintana-Murci et al., 2004 & 39 & 0 & 0 \\
\hline Barghuts & Derenko et al., 2012 & 149 & 4 & 2.68 \\
\hline Basque Country & Prieto et al., 2011 & 29 & 0 & 0 \\
\hline Basques from Northern Navarre & Cardoso et al., 2011 & 110 & 0 & 0 \\
\hline Belarusians & Kushniarevich et al., 2013 & 267 & 0 & 0 \\
\hline Borneo & Simonson et al., 2011 & 157 & 0 & 0 \\
\hline Bosnians & Malyarchuk et al., 2003 & 144 & 1 & 1 \\
\hline Brahui & Quintana-Murci et al., 2004 & 38 & 0 & 0 \\
\hline British people & Zheng et al., 2012 & 94 & 0 & 0 \\
\hline Bukharan Arabs & Comas et al., 2004 & 20 & 0 & 0 \\
\hline Bulgarians & Karachanak et al., 2012 & 855 & 1 & 0.12 \\
\hline Buryats & Derenko et al., 2007 & 295 & 4 & 1 \\
\hline Cantabria & Álvarez-Iglesias et al., 2010 & 135 & 0 & 0 \\
\hline Catalonia & Álvarez-Iglesias et al., 2010 & 101 & 0 & 0 \\
\hline Central Portugal & Prieto et al., 2011 & 53 & 0 & 0 \\
\hline Chaplin & Volodko et al., 2008 & 50 & 0 & 0 \\
\hline Chukchi & Gubina et al., 2013 & 159 & 0 & 0 \\
\hline Chukchi & Derenko et al., 2007 & 15 & 0 & 0 \\
\hline Chukchi & Volodko et al., 2008 & 182 & 0 & 0 \\
\hline Chutyr' & Grosheva et al., 2014 & 63 & 6 & 9.52 \\
\hline Crimean Tatars & Comas et al., 2004 & 20 & 0 & 0 \\
\hline Cypriots & Irwin et al., 2008 & 91 & 0 & 0 \\
\hline Czech & Mielnik-Sikorska et al., 2013 & 85 & 0 & 0 \\
\hline
\end{tabular}




\section{Continued}

Czechs (Western Bohemia)

Dai

Danes

Dong

Dungan

East Evenks

European Russia (Novgorod oblast)

Evenks

Ezhevo

Finns

Fins

French people

Gelao

Gilaki

Gujarati

Han Chinese

Hazara

Hui

Hunza Burusho

Hungarians (Budapest)

Iranians

Iranians

Iraqis

Java

Kabachigurt

Kalash

Kalmyks

Kazak

Kazak

Kazakhstan

Kazan Tatars (Azmakaevo)

Khakassians

Khamnigans

Khanty
Malyarchuk et al., 2006

Liu et al., 2011

Mikkelsen et al., 2010

Liu et al., 2011

Comas et al., 2004

Derenko et al., 2007

Grzybowski et al., 2007

Gubina et al., 2013

Grosheva et al., 2014

Hedman et al., 2007

Zheng et al., 2012

Richard et al., 2007

Liu et al., 2011

Quintana-Murci et al., 2004

Quintana-Murci et al., 2004

Yao et al., 2002

Quintana-Murci et al., 2004

Yao et al., 2004

Quintana-Murci et al., 2004

Irwin et al., 2007

Derenko et al., 2013

Comas et al., 2004

Al-Zahery et al., 2003

Simonson et al., 2011

Grosheva et al., 2014

Quintana-Murci et al., 2004

Derenko et al., 2007

Comas et al., 2004

Yao et al., 2004

Irwin et al., 2010

Malyarchuk et al., 2010

Derenko et al., 2007

Derenko et al., 2010

Pimenoff et al., 2008
179

41

201

28

16

45

157

38

39

200

97

868

177

37

34

263

23

45

44

211

352

20

216

46

57

44

110

20

53

256

71

57

99

106

0

0

0

3.57

0

0

0.64

3

3

0

0

0

2

0

0

2

13.04

4.44

0

0

0

0

0

0

1.75

0

2

5

11

1.17

2.82

0

0

0 


\section{Continued}

Khoremian Uzbek

Koreans

Koryaks

Kurdish-Western Iran

Kurdish-Turkmenistan

Kurds

Kuzyumovo

Kyrgyz

Kyrgyzstan

Lahu

Lesagurt

Lombok

Lower Kolyma-Indigirka

Lur

Macedonians (FYROM)

Makrani

Manchu

Mansi

Mazandarian

Miao

Mongolian

Mongolians

Mulam

Nanaians

Naukan

Naxi

Nganasan

Nivkhs

North and Central Italians

North Portugal

Northern Greeks

$\mathrm{Nu}$

Parsis

Pathan
Comas et al., 2004

Derenko et al., 2007

Gubina et al., 2013

Quintana-Murci et al., 2004

Quintana-Murci et al., 2004

Derenko et al., 2007

Grosheva et al., 2014

Comas et al., 2004

Irwin et al., 2010

Liu et al., 2011

Grosheva et al., 2014

Simonson et al., 2011

Volodko et al., 2008

Quintana-Murci et al., 2004

Zimmermann et al., 2007

Quintana-Murci et al., 2004

Zhao et al., 2011

Pimenoff et al., 2008

Quintana-Murci et al., 2004

Liu et al., 2011

Yao et al., 2004

Derenko et al., 2007

Liu et al., 2011

Gubina et al., 2013

Volodko et al., 2008

Liu et al., 2011

Volodko et al., 2008

Gubina et al., 2013

Turchi et al., 2008

Prieto et al., 2011

Irwin et al., 2008

Liu et al., 2011

Quintana-Murci et al., 2004

Quintana-Murci et al., 2004
20

103

69

20

32

25

72

20

249

35

63

44

82

17

200

33

47

63

21

103

49

47

39

86

39

45

39

17

395

187

319

30

44

44
0

0

21.74

0

0

0

16.67

0

1.20

3

9.52

0

2.44

0

0

0

2.13

0

0

0.97

0

2.13

2.56

0

0

2.22

2.56

0

0

0

0

16.67

0

0 


\section{Continued}

\begin{tabular}{|c|c|c|c|c|}
\hline Persian & Quintana-Murci et al., 2004 & 42 & 0 & 0 \\
\hline Persians & Derenko et al., 2013 & 181 & 0 & 0 \\
\hline Phillipines & Simonson et al., 2011 & 61 & 0 & 0 \\
\hline Poland (Kashubians) & Mielnik-Sikorska et al., 2013 & 203 & 0 & 0 \\
\hline Poland (Podhale) & Mielnik-Sikorska et al., 2013 & 201 & 0 & 0 \\
\hline Poland Northeastern & Grzybowski et al., 2007 & 73 & 0 & 0 \\
\hline Poland Northern & Grzybowski et al., 2007 & 253 & 0 & 0 \\
\hline Poland Southern & Grzybowski et al., 2007 & 87 & 0 & 0 \\
\hline Qashqais & Derenko et al., 2013 & 112 & 0 & 0 \\
\hline Saudi Arabs & Abu-Amero et al., 2008, 2007 & 553 & 0 & 0 \\
\hline Serbia-Vojvodina Province & Zgonjanin et al., 2010 & 104 & 0 & 0 \\
\hline Shors & Derenko et al., 2007 & 82 & 1 & 1.22 \\
\hline Shugnan & Quintana-Murci et al., 2004 & 44 & 0 & 0 \\
\hline Shui & Liu et al., 2011 & 30 & 0 & 0 \\
\hline Sindhi & Quintana-Murci et al., 2004 & 23 & 0 & 0 \\
\hline Sireniki & Volodko et al., 2008 & 37 & 0 & 0 \\
\hline Slovaks & Lehocký et al., 2008 & 374 & 0 & 0 \\
\hline Slovenians & Malyarchuk et al., 2003 & 104 & 0 & 0 \\
\hline Southern Germany (Ulm) & Brandsta"tter et al., 2006 & 100 & 0 & 0 \\
\hline Spain & Prieto et al., 2011 & 403 & 0 & 0 \\
\hline Staraya Salya & Grosheva et al., 2014 & 54 & 2 & 4 \\
\hline Sulawesi & Simonson et al., 2011 & 237 & 0 & 0 \\
\hline Sumatra & Simonson et al., 2011 & 180 & 1 & 0.56 \\
\hline Sumba & Simonson et al., 2011 & 50 & 0 & 0 \\
\hline Svetozarevo & Grosheva et al., 2014 & 45 & 4 & 8.89 \\
\hline Taiwan & Simonson et al., 2011 & 78 & 0 & 0 \\
\hline Tajik & Comas et al., 2004 & 20 & 0 & 0 \\
\hline Tajikistan & Irwin et al., 2010 & 244 & 0 & 0 \\
\hline Tajiks & Derenko et al., 2007 & 44 & 1 & 2.27 \\
\hline Tatars from Buinsk (Mishars) & Malyarchuk et al., 2010 & 126 & 0 & 0 \\
\hline Telenghits & Derenko et al., 2007 & 71 & 0 & 0 \\
\hline Teleuts & Derenko et al., 2007 & 53 & 3 & 5.66 \\
\hline Tibetan & Liu et al., 2011 & 40 & 0 & 0 \\
\hline Tsipya & Grosheva et al., 2014 & 53 & 4 & 7.55 \\
\hline Tujia & Liu et al., 2011 & 29 & 0 & 0 \\
\hline Turkish & Quintana-Murci et al., 2004 & 40 & 0 & 0 \\
\hline
\end{tabular}




\begin{tabular}{|c|c|c|c|c|}
\hline \multicolumn{5}{|l|}{ Continued } \\
\hline Turkmen & Comas et al., 2004 & 20 & 0 & 0 \\
\hline Turkmen & Quintana-Murci et al., 2004 & 41 & 0 & 0 \\
\hline Turkmenistan & Irwin et al., 2010 & 249 & 0 & 0 \\
\hline Tuscans & Zheng et al., 2012 & 105 & 0 & 0 \\
\hline Tuscans & Achilli et al., 2007 & 322 & 0 & 0 \\
\hline Tuvinians & Derenko et al., 2010 & 231 & 1 & 0.43 \\
\hline Uighur & Comas et al., 2004 & 16 & 0 & 0 \\
\hline Ukraine & Mielnik-Sikorska et al., 2013 & 159 & 0 & 0 \\
\hline Upper Anadyr (Chuvantsi) & Volodko et al., 2008 & 32 & 2 & 6.25 \\
\hline Upper Kolyma (Yukaghirs) & Volodko et al., 2008 & 18 & 1 & 5.56 \\
\hline Uygur & Yao et al., 2004 & 47 & 0 & 0 \\
\hline Uzbek & Comas et al., 2004 & 20 & 0 & 0 \\
\hline Uzbek & Yao et al., 2004 & 58 & 0 & 0 \\
\hline Uzbek & Quintana-Murci et al., 2004 & 42 & 0 & 0 \\
\hline Uzbekistan & Irwin et al., 2010 & 328 & 2 & 0.61 \\
\hline $\mathrm{Va}$ & Liu et al., 2011 & 36 & 0 & 0 \\
\hline West Evenks & Derenko et al., 2007 & 73 & 1 & 1.37 \\
\hline Yakuts & Derenko et al., 2010 & 254 & 0 & 0 \\
\hline Yao & Liu et al., 2011 & 40 & 1 & 3 \\
\hline $\mathrm{Yi}$ & Liu et al., 2011 & 20 & 0 & 0 \\
\hline Yugdon & Grosheva et al., 2014 & 58 & 1 & 1.72 \\
\hline Yukaghirs & Gubina et al., 2013 & 66 & 1 & 1.52 \\
\hline Zhulang & Liu et al., 2011 & 83 & 0 & 0 \\
\hline
\end{tabular}

\section{References S2(c)}

Abu-Amero, K. K., Gonzalez, A. M., Larruga, J. M., Bosley, T. M., \& Cabrera, V. M. (2007). Eurasian and African Mitochondrial DNA Influences in the Saudi Arabian Population. BMC Evolutionary Biology, 7, 32. http://dx.doi.org/10.1186/1471-2148-7-32

Abu-Amero, K. K., Larruga, J. M., Cabrera, V. M., \& Gonzalez, A. M. (2008). Mitochondrial DNA Structure in the Arabian Peninsula. BMC Evolutionary Biology, 8, 45. http://dx.doi.org/10.1186/1471-2148-8-45

Achilli, A., Olivieri, A., Pala, M., Metspalu, E., Fornarino, S., Battaglia, V. et al. (2007). Mitochondrial DNA Variation of Modern Tuscans Supports the Near Eastern Origin of Etruscans. American Journal of Human Genetics, 80, 759-768. http://dx.doi.org/10.1086/512822

Álvarez-Iglesias, V., Mosquera-Miguel, A., Cerezo, M., Quintáns, B., Zarrabeitia, M. T., Cuscó, I. et al. (2009). New Population and Phylogenetic Features of the Internal Variation within Mitochondrial DNA Macro-Haplogroup R0. PLoS ONE, 4, e5112. http://dx.doi.org/10.1371/journal.pone.0005112

Al-Zahery, N., Semino, O., Benuzzi, G., Magri, C., Passarino, G., Torroni, A., \& Santachiara-Benerecetti, A. S. (2003). Y-Chromosome and mtDNA Polymorphisms in Iraq, a Crossroad of the Early Human Dispersal and of Post-Neolithic Migrations. Molecular Phylogenetics and Evolution, 28, 458-472. http://dx.doi.org/10.1016/S1055-7903(03)00039-3

Brandstatter, A., Klein, R., Duftner, N., Wiegand, P., \& Parson, W. (2006). Application of a Quasi-Median Network 
Analysis for the Visualization of Character Conflicts to a Population Sample of Mitochondrial DNA Control Region Sequences from Southern Germany (Ulm). International Journal of Legal Medicine, 120, 310-314.

http://dx.doi.org/10.1007/s00414-006-0114-x

Brandstatter, A., Niederstatter, H., Pavlic, M., Grubwieser, P., \& Parson, W. (2007). Generating Population Data for the EMPOP Database-An Overview of the mtDNA Sequencing and Data Evaluation Processes Considering 273 Austrian Control Region Sequences as Example. Forensic Science International, 166, 164-175.

http://dx.doi.org/10.1016/j.forsciint.2006.05.006

Cardoso, S., Alfonso-Sanchez, M. A., Valverde, L., Odriozola, A., Perez-Miranda, A. M., Pena, J. A., \& de Pancorbo, M. M. (2011). The Maternal Legacy of Basques in Northern Navarre: New Insights into the Mitochondrial DNA Diversity of the Franco-Cantabrian Area. American Journal of Physical Anthropology, 145, 480-488. http://dx.doi.org/10.1002/ajpa.21532

Comas, D., Plaza, S., Wells, R. S., Yuldaseva, N., Lao, O., Calafell, F., \& Bertranpetit, J. (2004). Admixture, Migrations, and Dispersals in Central Asia: Evidence from Maternal DNA Lineages. European Journal of Human Genetics, 12, 495504. http://dx.doi.org/10.1038/sj.ejhg.5201160

Derenko, M., Malyarchuk, B., Bahmanimehr, A., Denisova, G., Perkova, M., Farjadian, S., \& Yepiskoposyan, L. (2013). Complete Mitochondrial DNA Diversity in Iranians. PLoS ONE, 8, e80673. http://dx.doi.org/10.1371/journal.pone.0080673

Derenko, M., Malyarchuk, B., Denisova, G., Perkova, M., Rogalla, U., Grzybowski, T. et al. (2012). Complete Mitochondrial DNA Analysis of Eastern Eurasian Haplogroups Rarely Found in Populations of Northern Asia and Eastern Europe. PLoS ONE, 7, e32179. http://dx.doi.org/10.1371/journal.pone.0032179

Derenko, M., Malyarchuk, B., Grzybowski, T., Denisova, G., Dambueva, I., Perkova, M. et al. (2007). Phylogeographic Analysis of Mitochondrial DNA in Northern Asian Populations. The American Journal of Human Genetics, 81, 10251041. http://dx.doi.org/10.1086/522933

Derenko, M., Malyarchuk, B., Grzybowski, T., Denisova, G., Rogalla, U., Perkova, M. et al. (2010). Origin and Post-Glacial Dispersal of Mitochondrial DNA Haplogroups C and D in Northern Asia. PLoS ONE, 5, e15214. http://dx.doi.org/10.1371/journal.pone.0015214

Grosheva, A., Shneider, Y. V., Zhukova, O., Morozova, I. Y., \& Rychkov, S. Y. (2014). Features of the Udmurt Mitochondrial Gene Pool in Relation to Tribal Structure. Russian Journal of Genetics, 50, 975-986. http://dx.doi.org/10.1134/S1022795414090063

Grzybowski, T., Malyarchuk, B. A., Derenko, M. V., Perkova, M. A., Bednarek, J., \& Wozniak, M. (2007). Complex Interactions of the Eastern and Western Slavic Populations with Other European Groups as Revealed by Mitochondrial DNA Analysis. Forensic Science International: Genetics, 1, 141-147. http://dx.doi.org/10.1016/j.fsigen.2007.01.010

Gubina, M. A., Girgol'kau, L. A., Babenko, V. N., Damba, L. D., Maksimov, V. N., \& Voevoda, M. I. (2013). Mitochondrial DNA Polymorphism in Populations of Aboriginal Residents of the Far East. Russian Journal of Genetics, 49, 751-764. http://dx.doi.org/10.1134/S1022795413070065

Hedman, M., Brandstätter, A., Pimenoff, V., Sistonen, P., Palo, J. U., Parson, W., \& Sajantila, A. (2007). Finnish Mitochondrial DNA HVS-I and HVS-II Population Data. Forensic Science International, 172, 171-178. http://dx.doi.org/10.1016/j.forsciint.2006.09.012

Irwin, J. A., Ikramov, A., Saunier, J., Bodner, M., Amory, S., Rock, A. et al. (2010). The mtDNA Composition of Uzbekistan: A Microcosm of Central Asian Patterns. International Journal of Legal Medicine, 124, 195-204. http://dx.doi.org/10.1007/s00414-009-0406-z

Irwin, J., Egyed, B., Saunier, J., Szamosi, G., O’Callaghan, J., Padar, Z., \& Parsons, T. (2007). Hungarian mtDNA Population Databases from Budapest and the Baranya County Roma. International Journal of Legal Medicine, 121, 377383. http://dx.doi.org/10.1007/s00414-006-0128-4

Irwin, J., Saunier, J., Strouss, K., Paintner, C., Diegoli, T., Sturk, K. et al. (2008). Mitochondrial Control Region Sequences from Northern Greece and Greek Cypriots. International Journal of Legal Medicine, 122, 87-89.

http://dx.doi.org/10.1007/s00414-007-0173-7

Karachanak, S., Carossa, V., Nesheva, D., Olivieri, A., Pala, M., Hooshiar Kashani, B. et al. (2012). Bulgarians vs the Other European Populations: A Mitochondrial DNA Perspective. International Journal of Legal Medicine, 126, 497-503. http://dx.doi.org/10.1007/s00414-011-0589-y

Kushniarevich, A., Sivitskaya, L., Danilenko, N., Novogrodskii, T., Tsybovsky, I., Kiseleva, A. et al. (2013). Uniparental Genetic Heritage of Belarusians: Encounter of Rare Middle Eastern Matrilineages with a Central European Mitochondrial DNA Pool. PLoS ONE, 8, e66499. http://dx.doi.org/10.1371/journal.pone.0066499

Lehocký, I., Baldovič, M., Kádaši, L., \& Metspalu, E. (2008). A Database of Mitochondrial DNA Hypervariable Regions I and II Sequences of Individuals from Slovakia. Forensic Science International: Genetics, 2, e53-e59. http://dx.doi.org/10.1016/j.fsigen.2007.12.008 
Liu, C., Wang, S.-Y., Zhao, M., Xu, Z.-Y., Hu, Y.-H., Chen, F. et al. (2011). Mitochondrial DNA Polymorphisms in Gelao Ethnic Group Residing in Southwest China. Forensic Science International: Genetics, 5, e4-e10. http://dx.doi.org/10.1016/j.fsigen.2010.04.007

Malyarchuk, B. A., Grzybowski, T., Derenko, M. V., Czarny, J., Drobnič, K., \& Miścicka-Śliwka, D. (2003). Mitochondrial DNA Variability in Bosnians and Slovenians. Annals of Human Genetics, 67, 412-425. http://dx.doi.org/10.1046/j.1469-1809.2003.00042.x

Malyarchuk, B. A., Vanecek, T., Perkova, M. A., Derenko, M. V., \& Sip, M. (2006). Mitochondrial DNA Variability in the Czech Population, with Application to the Ethnic History of Slavs. Human Biology, 78, 681-696. http://dx.doi.org/10.1353/hub.2007.0014

Malyarchuk, B., Derenko, M., Denisova, G., \& Kravtsova, O. (2010). Mitogenomic Diversity in Tatars from the Volga-Ural Region of Russia. Molecular Biology and Evolution, 27, 2220-2226. http://dx.doi.org/10.1093/molbev/msq065

Mielnik-Sikorska, M., Daca, P., Malyarchuk, B., Derenko, M., Skonieczna, K., Perkova, M. et al. (2013). The History of Slavs Inferred from Complete Mitochondrial Genome Sequences. PLoS ONE, 8, e54360. http://dx.doi.org/10.1371/journal.pone.0054360

Mikkelsen, M., Sorensen, E., Rasmussen, E. M., \& Morling, N. (2010). Mitochondrial DNA HV1 and HV2 Variation in Danes. Forensic Science International: Genetics, 4, e87-88. http://dx.doi.org/10.1016/j.fsigen.2009.07.007

Pimenoff, V. N., Comas, D., Palo, J. U., Vershubsky, G., Kozlov, A., \& Sajantila, A. (2008). Northwest Siberian Khanty and Mansi in the junction of West and East Eurasian Gene Pools as Revealed by Uniparental Markers. European Journal of Human Genetics, 16, 1254-1264. http://www.nature.com/ejhg/journal/v16/n10/suppinfo/ejhg2008101s1.html http://dx.doi.org/10.1038/ejhg.2008.101

Prieto, L., Zimmermann, B., Goios, A., Rodriguez-Monge, A., Paneto, G. G., Alves, C. et al. (2011). The GHEP-EMPOP Collaboration on mtDNA Population Data-A New Resource for Forensic Casework. Forensic Science International: Genetics, 5, 146-151. http://dx.doi.org/10.1016/j.fsigen.2010.10.013

Quintana-Murci, L., Chaix, R., Wells, R. S., Behar, D. M., Sayar, H., Scozzari, R. et al. (2004). Where West Meets East: The Complex mtDNA Landscape of the Southwest and Central Asian Corridor. American Journal of Human Genetics, 74, 827-845. http://dx.doi.org/10.1086/383236

Richard, C., Pennarun, E., Kivisild, T., Tambets, K., Tolk, H. V., Metspalu, E. et al. (2007). An mtDNA Perspective of French Genetic Variation. Annals of Human Biology, 34, 68-79. http://dx.doi.org/10.1080/03014460601076098

Simonson, T. S., Xing, J., Barrett, R., Jerah, E., Loa, P., Zhang, Y. et al. (2011). Ancestry of the Iban Is Predominantly Southeast Asian: Genetic Evidence from Autosomal, Mitochondrial, and Y Chromosomes. PLoS ONE, 6, e16338. http://dx.doi.org/10.1371/journal.pone.0016338

Turchi, C., Buscemi, L., Previdere, C., Grignani, P., Brandstatter, A., Achilli, A. et al. (2008). Italian Mitochondrial DNA Database: Results of a Collaborative Exercise and Proficiency Testing. International Journal of Legal Medicine, 122, 199204. http://dx.doi.org/10.1007/s00414-007-0207-1

Volodko, N. V., Starikovskaya, E. B., Mazunin, I. O., Eltsov, N. P., Naidenko, P. V., Wallace, D. C., \& Sukernik, R. I. (2008). Mitochondrial Genome Diversity in Arctic Siberians, with Particular Reference to the Evolutionary History of Beringia and Pleistocenic Peopling of the Americas. The American Journal of Human Genetics, 82, 1084-1100. http://dx.doi.org/10.1016/j.ajhg.2008.03.019

Yao, Y. G., Kong, Q. P., Wang, C. Y., Zhu, C. L., \& Zhang, Y. P. (2004). Different Matrilineal Contributions to Genetic Structure of Ethnic Groups in the Silk Road Region in China. Molecular Biology and Evolution, 21, 2265-2280. http://dx.doi.org/10.1093/molbev/msh238

Yao, Y.-G., Kong, Q.-P., Bandelt, H.-J., Kivisild, T. et al. (2002). Phylogeographic Differentiation of Mitochondrial DNA in Han Chinese. The American Journal of Human Genetics, 70, 635-651. http://dx.doi.org/10.1086/338999

Zgonjanin, D., Veselinovic, I., Kubat, M., Furac, I., Antov, M., Loncar, E., \& Omorjan, R. (2010). Sequence Polymorphism of the Mitochondrial DNA Control Region in the Population of Vojvodina Province, Serbia. Legal medicine (Tokyo, Japan), 12, 104-107. http://dx.doi.org/10.1016/j.legalmed.2009.10.007

Zhao, Y. B., Sun, W. Y., Zhan, Y., Di, W., \& Yu, C. C. (2011). Mitochondrial DNA Evidence of Southward Migration of Manchus in China. Molekuliarnaia Biologiia (Moskva), 45, 825-830. http://dx.doi.org/10.1134/s0026893311050153

Zheng, H.-X., Yan, S., Qin, Z.-D., \& Jin, L. (2012). MtDNA Analysis of Global Populations Support That Major Population Expansions Began before Neolithic Time. Scientific Reports, 2, 745. http://dx.doi.org/10.1038/srep00745

Zimmermann, B., Brandstatter, A., Duftner, N., Niederwieser, D., Spiroski, M., Arsov, T., \& Parson, W. (2007). Mitochondrial DNA Control Region Population Data from Macedonia. Forensic Science International: Genetics, 1, e4-e9.

http://dx.doi.org/10.1016/j.fsigen.2007.03.002 
Table S3. Absolute frequencies of Y-chromosome haplogroups $\mathrm{C}, \mathrm{N}$ and $\mathrm{Q}$ in the population groups used in the chi-square test comparison. N-number of analyzed individuals; ${ }^{*}$-value population group $v s$ Bulgarians.

\begin{tabular}{|c|c|c|c|c|c|c|}
\hline Population group & $\mathrm{N}$ & $\mathrm{Hg} \mathrm{C}$ & $\mathrm{Hg} \mathrm{N}$ & $\mathrm{Hg} \mathrm{Q}$ & Reference & $p^{*}$ \\
\hline \multicolumn{7}{|l|}{ Altai populations } \\
\hline Altaian Kazakhs (Southeastern) & 89 & 54 & 0 & 0 & Dulik et al., 2012 & \\
\hline Altai & 98 & 22 & 4 & 17 & Hammer et al., 2006 & \\
\hline Altaian Kazakhs (Southwestern) & 30 & 17 & 0 & 1 & Dulik et al., 2012 & \\
\hline Altaians & 59 & 5 & 19 & 5 & Gubina et al., 2013 & \\
\hline Altai-Kizhi & 120 & 24 & 5 & 20 & Dulik et al., 2012 & \\
\hline Chelkan & 25 & 0 & 5 & 15 & Dulik et al., 2012 & \\
\hline Kumandin & 17 & 0 & 9 & 0 & Dulik et al., 2012 & \\
\hline Tubalar & 27 & 0 & 3 & 10 & Dulik et al., 2012 & \\
\hline Total & 465 & 122 & 45 & 68 & & $<0.0001$ \\
\hline \multicolumn{7}{|l|}{$\underline{\text { Siberian populations }}$} \\
\hline Evenks & 152 & 83 & 52 & 4 & Hammer et al., 2006; Fedorova et al., 2013 & \\
\hline Buryats & 298 & 119 & 143 & 0 & Kharkov et al., 2014 & \\
\hline Evens & 24 & 10 & 12 & 0 & Fedorova et al., 2013 & \\
\hline Shorians & 21 & 0 & 17 & 0 & Gubina et al., 2013 & \\
\hline Tuvinians & 102 & 27 & 46 & 5 & Gubina et al., 2013 & \\
\hline Yakuts & 234 & 14 & 205 & 0 & Sengupta et al., 2006; Fedorova et al., 2013 & \\
\hline Yukaghirs & 11 & 4 & 3 & 0 & Fedorova et al., 2013 & \\
\hline Total & 842 & 257 & 478 & 9 & & $<0.00001$ \\
\hline \multicolumn{7}{|l|}{$\underline{\text { Central Asian populations }}$} \\
\hline Khakassians & 176 & 2 & 88 & 7 & Gubina et al., 2013 & \\
\hline Kazakhs & 49 & 20 & 2 & 0 & Gubina et al., 2013 & \\
\hline Kyrgyz & 132 & 26 & 6 & 1 & Di Cristofaro et al., 2013 & \\
\hline Tajik & 142 & 13 & 5 & 5 & Di Cristofaro et al., 2013 & \\
\hline Turkmen-Jawzjan & 74 & 1 & 5 & 25 & Di Cristofaro et al., 2013 & \\
\hline Uzbek & 127 & 5 & 5 & 11 & Di Cristofaro et al., 2013 & \\
\hline Total & 700 & 67 & 111 & 49 & & $<0.00001$ \\
\hline \multicolumn{7}{|l|}{$\underline{\text { Europeans }}$} \\
\hline Belarussian-East Polesie & 96 & 0 & 8 & 0 & Kushniarevich et al., 2013 & \\
\hline Belarussian-West Polesie & 121 & 0 & 10 & 1 & Kushniarevich et al., 2013 & \\
\hline Belarussian-Centre & 88 & 0 & 7 & 0 & Kushniarevich et al., 2013 & \\
\hline Belarussian-East & 86 & 0 & 6 & 0 & Kushniarevich et al., 2013 & \\
\hline Belarussian-North & 101 & 0 & 16 & 0 & Kushniarevich et al., 2013 & \\
\hline Belarussian-West & 73 & 0 & 10 & 0 & Kushniarevich et al., 2013 & \\
\hline Bosniacs & 84 & 0 & 0 & 0 & Battaglia et al., 2009 & \\
\hline Bosnian Croats & 90 & 0 & 0 & 0 & Battaglia et al., 2009 & \\
\hline Bosnian Serbs & 81 & 0 & 5 & 0 & Battaglia et al., 2009 & \\
\hline Corsica & 34 & 0 & 0 & 0 & Francalacci et al., 2003 & \\
\hline
\end{tabular}




\section{Continued}

\begin{tabular}{|c|c|c|c|c|c|c|}
\hline Croats & 89 & 0 & 2 & 0 & Battaglia et al., 2009 & \\
\hline Croats-Osijek & 29 & 0 & 0 & 0 & Battaglia et al., 2009 & \\
\hline Czechs & 75 & 0 & 2 & 0 & Battaglia et al., 2009 & \\
\hline France & 23 & 0 & 0 & 0 & Semino et al., 2000 & \\
\hline FYROM & 211 & 0 & 1 & 0 & Noveski et al., 2010 & \\
\hline Germany & 16 & 0 & 0 & 0 & Semino et al., 2000 & \\
\hline Gipuzkoa Basques & 74 & 0 & 0 & 0 & Alonso et al., 2005 & \\
\hline Greece & 92 & 1 & 0 & 0 & Battaglia et al., 2009 & \\
\hline Hungary & 53 & 0 & 0 & 0 & Battaglia et al., 2009 & \\
\hline Italy-Central Marche & 59 & 0 & 0 & 0 & Capelli et al., 2007 & \\
\hline Italy-Elba Island (Tuscany) & 95 & 0 & 0 & 0 & Capelli et al., 2007 & \\
\hline Macedonian Greeks & 57 & 0 & 0 & 0 & Battaglia et al., 2009 & \\
\hline Poland & 99 & 0 & 1 & 0 & Battaglia et al., 2009 & \\
\hline Portugal-Central & 102 & 0 & 0 & 0 & Goncalves et al., 2005 & \\
\hline Portugal-North & 101 & 1 & 0 & 0 & Goncalves et al., 2005 & \\
\hline Romania & 149 & 0 & 1 & 1 & Martinez-Cruz et al., 2012 & \\
\hline Serbia & 103 & 0 & 2 & 0 & Regueiro et al., 2012 & \\
\hline Slovenia & 75 & 0 & 0 & 0 & Battaglia et al., 2009 & \\
\hline Spain-Cinco Villas & 42 & 0 & 0 & 0 & Lopez-Parra et al., 2009 & \\
\hline Spain-Jacetania & 31 & 0 & 0 & 0 & Lopez-Parra et al., 2009 & \\
\hline Spain-Vallede Ar'an & 25 & 0 & 0 & 0 & Lopez-Parra et al., 2009 & \\
\hline Spain-Alto Urgel & 34 & 1 & 0 & 0 & Lopez-Parra et al., 2009 & \\
\hline Spain-Cerda na & 37 & 1 & 0 & 0 & Lopez-Parra et al., 2009 & \\
\hline Ukraine & 92 & 0 & 6 & 1 & Battaglia et al., 2009 & \\
\hline Total & 2617 & 4 & 77 & 3 & & 0.000035 \\
\hline \multicolumn{7}{|l|}{$\frac{\text { European neighbouring }}{\underline{\text { countries of Bulgaria }}}$} \\
\hline FYROM & 211 & 0 & 1 & 0 & Noveski et al., 2010 & \\
\hline Macedonian Greeks & 57 & 0 & 0 & 0 & Battaglia et al., 2009 & \\
\hline Serbia & 103 & 0 & 2 & 0 & Regueiro et al., 2012 & \\
\hline Romanians & 149 & 0 & 1 & 1 & Martinez-Cruz et al., 2012 & \\
\hline Total & 520 & 0 & 4 & 1 & & 0.289988 \\
\hline Bulgarians & 808 & 4 & 4 & 4 & Karachanak et al., 2013 & \\
\hline
\end{tabular}

\section{References S3}

Abu-Amero, Alonso, S., Flores, C., Cabrera, V., Alonso, A., Martin, P., Albarran, C. et al. (2005). The Place of the Basques in the European Y-Chromosome Diversity Landscape. European Journal of Human Genetics, 13, 1293-1302.

http://dx.doi.org/10.1038/sj.ejhg.5201482 
Battaglia, V., Fornarino, S., Al-Zahery, N., Olivieri, A., Pala, M., Myres, N. M. et al. (2009). Y-Chromosomal Evidence of the Cultural Diffusion of Agriculture in Southeast Europe. European Journal of Human Genetics, 17, 820-830. http://dx.doi.org/10.1038/ejhg.2008.249

Capelli, C., Redhead, N., Abernethy, J. K., Gratrix, F., Wilson, J. F., Moen, T. et al. (2003). A Y Chromosome Census of the British Isles. Current Biology, 13, 979-984. http://dx.doi.org/10.1016/s0960-9822(03)00373-7

Di Cristofaro, J., Pennarun, E., Mazières, S., Myres, N. M., Lin, A. A., Temori, S. A. et al. (2013). Afghan Hindu Kush: Where Eurasian Sub-Continent Gene Flows Converge. PLoS ONE, 8, e76748. http://dx.doi.org/10.1371/journal.pone.0076748

Dulik, M. C., Osipova, L. P., \& Schurr, T. G. (2011). Y-Chromosome Variation in Altaian Kazakhs Reveals a Common Paternal Gene Pool for Kazakhs and the Influence of Mongolian Expansions. PLoS ONE, 6, e17548. http://dx.doi.org/10.1371/journal.pone.0017548

Dulik, M. C., Zhadanov, S. I., Osipova, L. P., Askapuli, A., Gau, L., Gokcumen, O. et al. (2012). Mitochondrial DNA and Y Chromosome Variation Provides Evidence for a Recent Common Ancestry between Native Americans and Indigenous Altaians. American Journal of Human Genetics, 90, 229-246. http://dx.doi.org/10.1016/j.ajhg.2011.12.014

Fedorova, S. A., Reidla, M., Metspalu, E., Metspalu, M., Rootsi, S., Tambets, K. et al. (2013). Autosomal and Uniparental Portraits of the Native Populations of Sakha (Yakutia): Implications for the Peopling of Northeast Eurasia. BMC Evolutionary Biology, 13, 127. http://dx.doi.org/10.1186/1471-2148-13-127

Francalacci, P., Morelli, L., Underhill, P. A., Lillie, A. S., Passarino, G., Useli, A. et al. (2003). Peopling of Three Mediterranean Islands (Corsica, Sardinia, and Sicily) Inferred by Y-Chromosome Biallelic Variability. American Journal of Physical Anthropology, 121, 270-279. http://dx.doi.org/10.1002/ajpa.10265

Gonçalves, R., Freitas, A., Branco, M., Rosa, A., Fernandes, A. T., Zhivotovsky, L. A. et al. (2005). Y-Chromosome Lineages from Portugal, Madeira and Açores Record Elements of Sephardim and Berber Ancestry. Annals of Human Genetics, 69, 443-454. http://dx.doi.org/10.1111/j.1529-8817.2005.00161.x

Gubina, M. A., Damba, L. D., Babenko, V. N., Romaschenko, A. G., \& Voevoda, M. I. (2013). Haplotype Diversity in mtDNA and Y-Chromosome in Populations of Altai-Sayan Region. Russian Journal of Genetics, 49, 329-343. http://dx.doi.org/10.1134/S1022795412120034

Hammer, M. F., Karafet, T. M., Park, H., Omoto, K., Harihara, S., Stoneking, M., \& Horai, S. (2005). Dual Origins of the Japanese: Common Ground for Hunter-Gatherer and Farmer Y Chromosomes. Journal of Human Genetics, 51, 47-58. http://dx.doi.org/10.1007/s10038-005-0322-0

Karachanak, S., Grugni, V., Fornarino, S., Nesheva, D., Al-Zahery, N., Battaglia, V. et al. (2013). Y-Chromosome Diversity in Modern Bulgarians: New Clues about Their Ancestry. PLoS ONE, 8, e 56779.

http://dx.doi.org/10.1371/journal.pone.0056779

Kharkov, V. N., Khamina, K. V., Medvedeva, O. F., Simonova, K. V., Eremina, E. R., \& Stepanov, V. A. (2014). Gene Pool of Buryats: Clinal Variability and Territorial Subdivision Based on Data of Y-Chromosome Markers. Russian Journal of Genetics, 50, 180-190. http://dx.doi.org/10.1134/S1022795413110082

Kushniarevich, A., Sivitskaya, L., Danilenko, N., Novogrodskii, T., Tsybovsky, I., Kiseleva, A. et al. (2013). Uniparental Genetic Heritage of Belarusians: Encounter of Rare Middle Eastern Matrilineages with a Central European Mitochondrial DNA Pool. PLoS ONE, 8, e66499. http://dx.doi.org/10.1371/journal.pone.0066499

Lacau, H., Gayden, T., Regueiro, M., Chennakrishnaiah, S., Bukhari, A., Underhill, P. A. et al. (2012). Afghanistan from a Y-Chromosome Perspective. European Journal of Human Genetics, 20, 1063-1070. http://dx.doi.org/10.1038/ejhg.2012.59

López-Parra, A., Gusmao, L., Tavares, L., Baeza, C., Amorim, A., Mesa, M. et al. (2009). In search of the Pre- and Post-Neolithic Genetic Substrates in Iberia: Evidence from Y-Chromosome in Pyrenean Populations. Annals of Human Genetics, 73, 42-53. http://dx.doi.org/10.1111/j.1469-1809.2008.00478.x

Martinez-Cruz, B., Ioana, M., Calafell, F., Arauna, L. R., Sanz, P., Ionescu, R. et al. (2012). Y-Chromosome Analysis in Individuals Bearing the Basarab Name of the First Dynasty of Wallachian Kings. PLoS ONE, 7, e41803. http://dx.doi.org/10.1371/journal.pone.0041803

Noveski, P., Trivodalieva, S., Efremov, G., \& Plaseska-Karanfilska, D. (2010). Y Chromosome Single Nucleotide Polymorphisms Typing by SNaPshot Minisequencing. Balkan Journal of Medical Genetics, 13, 9-16. http://dx.doi.org/10.2478/v10034-010-0013-9

Regueiro, M., Rivera, L., Damnjanovic, T., Lukovic, L., Milasin, J., \& Herrera, R. J. (2012). High Levels of Paleolithic Y-Chromosome Lineages Characterize Serbia. Gene, 498, 59-67. http://dx.doi.org/10.1016/j.gene.2012.01.030

Semino, O., Passarino, G., Oefner, P. J., Lin, A. A., Arbuzova, S., Beckman, L. E. et al. (2000). The Genetic Legacy of Paleolithic Homo Sapiens Sapiens in Extant Europeans: A Y Chromosome Perspective. Science, 290, 1155-1159. http://dx.doi.org/10.1126/science.290.5494.1155 
Table S4. Absolute frequencies of mtDNA haplogroups C and D in the population groups used in the chi-square test comparison. N-number of analyzed individuals; ${ }^{*} p$-value population group $v s$ Bulgarians

\begin{tabular}{|c|c|c|c|c|c|}
\hline Population group & $\mathrm{N}$ & $\mathrm{Hg} \mathrm{C}$ & Hg D & Reference & $p^{*}$ \\
\hline \multicolumn{6}{|l|}{ Altai populations } \\
\hline Altaian Kazakhs & 98 & 8 & 26 & Derenko et al., 2012 & \\
\hline Altaians & 110 & 21 & 17 & Derenko et al., 2010 & \\
\hline Altai-Kizhi & 324 & 98 & 58 & Dulik et al., 2012 & \\
\hline Chelkan & 91 & 14 & 21 & Dulik et al., 2012 & \\
\hline Kumandin & 52 & 22 & 10 & Dulik et al., 2012 & \\
\hline Shor & 28 & 5 & 8 & Dulik et al., 2012 & \\
\hline Telenghits & 71 & 12 & 15 & Derenko et al., 2010 & \\
\hline Telengit & 55 & 8 & 10 & Dulik et al., 2012 & \\
\hline Teleuts & 53 & 15 & 13 & Derenko et al., 2010 & \\
\hline Teleuts & 33 & 8 & 9 & Dulik et al., 2012 & \\
\hline Tubalar & 143 & 41 & 36 & Dulik et al., 2012 & \\
\hline Total & 1058 & 252 & 223 & & $<0.0001$ \\
\hline \multicolumn{6}{|l|}{$\underline{\text { Siberian populations }}$} \\
\hline Buryats & 419 & 89 & 146 & Derenko et al., 2010 & \\
\hline Dolgans & 157 & 61 & 62 & Derenko et al., 2010 & \\
\hline East Evenks & 45 & 29 & 11 & Derenko et al., 2010 & \\
\hline Evenks & 109 & 71 & 26 & Derenko et al., 2010; Gubina et al., 2013 & \\
\hline Kalmyks & 110 & 12 & 32 & Derenko et al., 2010 & \\
\hline Kets & 104 & 13 & 3 & Derenko et al., 2010 & \\
\hline Khakassians & 110 & 30 & 16 & Derenko et al., 2010 & \\
\hline Khamnigans & 99 & 16 & 31 & Derenko et al., 2010 & \\
\hline Khants & 106 & 11 & 17 & Derenko et al., 2010 & \\
\hline Mansi & 161 & 30 & 20 & Derenko et al., 2010 & \\
\hline Nenets & 58 & 18 & 13 & Derenko et al., 2010 & \\
\hline Nenets Tundra & 70 & 34 & 4 & Derenko et al., 2010 & \\
\hline Nganasans & 78 & 26 & 22 & Derenko et al., 2010 & \\
\hline Nganasans & 78 & 26 & 22 & Derenko et al., 2010 & \\
\hline Selkups & 120 & 26 & 4 & Derenko et al., 2010 & \\
\hline Shors & 82 & 10 & 10 & Derenko et al., 2010 & \\
\hline Sojots & 30 & 6 & 14 & Derenko et al., 2010 & \\
\hline Todjins & 48 & 23 & 2 & Derenko et al., 2010 & \\
\hline Tofalars & 58 & 36 & 0 & Derenko et al., 2010 & \\
\hline Tuvinians & 231 & 109 & 36 & Derenko et al., 2010 & \\
\hline
\end{tabular}




\section{Continued}

\begin{tabular}{|c|c|c|c|c|c|}
\hline West Evenks & 73 & 34 & 22 & Derenko et al., 2010 & \\
\hline Yakuts & 254 & 119 & 15 & Derenko et al., 2010 & \\
\hline Total & 2600 & 829 & 528 & & $<0.00001$ \\
\hline \multicolumn{6}{|l|}{$\underline{\text { Central Asian populations }}$} \\
\hline Bukharan Arabs & 20 & 4 & 0 & Comas et al., 2004 & \\
\hline Karakalpaks & 108 & 16 & 19 & Derenko et al., 2010 & \\
\hline Kazak & 840 & 72 & 155 & $\begin{array}{l}\text { Derenko et al., 2010; Irwin et al., 2010; } \\
\text { Comas et al., 2004; Yao et al., } 2004\end{array}$ & \\
\hline Kyrgyz & 469 & 66 & 90 & $\begin{array}{l}\text { Derenko et al., 2010; Irwin et al., 2010; } \\
\text { Comas et al., } 2004\end{array}$ & \\
\hline Tajiks & 414 & 58 & 28 & $\begin{array}{c}\text { Derenko et al., 2010; Irwin et al., 2010; } \\
\text { Quintana-Murci et al., } 2004\end{array}$ & \\
\hline Turkmens & 447 & 42 & 61 & $\begin{array}{l}\text { Derenko et al., 2010; Irwin et al., 2010; } \\
\text { Comas et al., } 2004\end{array}$ & \\
\hline Uighurs & 224 & 16 & 29 & Derenko et al., 2010; Yao et al. 2004 & \\
\hline Uzbeks & 556 & 40 & 62 & $\begin{array}{l}\text { Derenko et al., 2010; Irwin et al., 2010; } \\
\text { Comas et al., 2004; Yao et al. } 2004\end{array}$ & \\
\hline Total & 3078 & 314 & 444 & & $<0.0001$ \\
\hline \multicolumn{6}{|l|}{ Caucasus } \\
\hline Azerbaijanians & 89 & 6 & 0 & Derenko et al., 2010 & \\
\hline Chechens & 67 & 6 & 0 & Derenko et al., 2010 & \\
\hline Darginians & 37 & 2 & 0 & Derenko et al., 2010 & \\
\hline Georgians & 57 & 2 & 6 & Derenko et al., 2010 & \\
\hline Kabardinians & 51 & 2 & 0 & Derenko et al., 2010 & \\
\hline Nogays & 206 & 50 & 32 & Derenko et al., 2010 & \\
\hline Northern Caucasians & 199 & 16 & 16 & Derenko et al., 2010 & \\
\hline Osettins & 123 & 8 & 0 & Derenko et al., 2010 & \\
\hline Total & 829 & 92 & 54 & & $<0.00001$ \\
\hline Austrians & 273 & 1 & 0 & Brandsta“tter et al., 2007 & \\
\hline Basque Country & 29 & 0 & 0 & Prieto et al., 2011 & \\
\hline Basques from Northern Navarre & 110 & 0 & 0 & Cardoso et al., 2011 & \\
\hline Belarusians & 267 & 3 & 1 & Kushniarevich et al., 2013 & \\
\hline Bosnians & 144 & 0 & 0 & Malyarchuk et al., 2003 & \\
\hline British people & 94 & 0 & 0 & Zheng et al., 2012 & \\
\hline Cantabria & 135 & 0 & 0 & Álvarez-Iglesias et al., 2009 & \\
\hline Catalonia & 101 & 0 & 2 & Álvarez-Iglesias et al., 2009 & \\
\hline Central Portugal & 53 & 0 & 0 & Prieto et al., 2011 & \\
\hline Cypriots & 91 & 0 & 0 & Irwin et al., 2008 & \\
\hline Czech & 85 & 0 & 0 & Mielnik-Sikorska et al., 2013 & \\
\hline Czechs (Western Bohemia) & 179 & 0 & 1 & Malyarchuk et al., 2006 & \\
\hline
\end{tabular}




\begin{tabular}{|c|c|c|c|c|c|}
\hline Continued & & & & & \\
\hline Danes & 201 & 0 & 0 & Mikkelsen et al., 2010 & \\
\hline European Russia (Novgorod oblast) & 157 & 0 & 2 & Grzybowski et al., 2007 & \\
\hline Fins & 297 & 0 & 0 & Zheng et al., 2012; Hedman et al., 2007 & \\
\hline French people & 868 & 0 & 0 & Richard et al., 2007 & \\
\hline Hungarians & 344 & 2 & 2 & Derenko et al., 2010 & \\
\hline Hungarians (Budapest) & 211 & 0 & 2 & Irwin et al., 2007 & \\
\hline Macedonians (FYROM) & 200 & 0 & 0 & Zimmermann et al., 2007 & \\
\hline North and Central Italians & 395 & 0 & 3 & Turchi et al., 2008 & \\
\hline North Portugal & 187 & 0 & 1 & Prieto et al., 2011 & \\
\hline Northern Greeks & 319 & 1 & 0 & Irwin et al., 2008 & \\
\hline Poland (Kashubians) & 203 & 1 & 0 & Mielnik-Sikorska et al., 2013 & \\
\hline Poland (Podhale) & 201 & 0 & 1 & Mielnik-Sikorska et al., 2013 & \\
\hline Poland Northeastern & 73 & 0 & 0 & Grzybowski et al., 2007 & \\
\hline Poland Northern & 253 & 0 & 0 & Grzybowski et al., 2007 & \\
\hline Poland Southern & 87 & 1 & 0 & Grzybowski et al., 2007 & \\
\hline Poles & 947 & 5 & 3 & Derenko et al., 2010 & \\
\hline Serbia & 139 & 0 & 1 & Davidovic et al., 2014 & \\
\hline Serbia-Vojvodina Province & 104 & 0 & 0 & Zgonjanin et al., 2010 & \\
\hline Slovaks & 374 & 4 & 0 & Lehocký et al., 2008 & \\
\hline Slovenians & 104 & 0 & 0 & Malyarchuk et al., 2003 & \\
\hline Southern Germany (Ulm) & 100 & 0 & 0 & Brandsta"tter et al., 2006 & \\
\hline Spain & 403 & 0 & 0 & Prieto et al., 2011 & \\
\hline Tuscans & 105 & 0 & 1 & Zheng et al., 2012 & \\
\hline Tuscans & 322 & 0 & 1 & Achilli et al., 2007 & \\
\hline Ukraine & 159 & 2 & 0 & Mielnik-Sikorska et al., 2013 & \\
\hline Total & 8229 & 20 & 21 & & 0.873 \\
\hline \multicolumn{6}{|l|}{$\begin{array}{l}\text { European neighbouring } \\
\text { countries of Bulgaria }\end{array}$} \\
\hline FYROM & 200 & 0 & 0 & Zimmermann et al., 2007 & \\
\hline Northern Greeks & 319 & 1 & 0 & Irwin et al., 2008 & \\
\hline Serbia & 139 & 0 & 1 & Davidovic et al., 2014 & \\
\hline Total & 658 & 1 & 1 & & 0.710 \\
\hline Bulgarians & 2 & 3 & 850 & Karachanak et al., 2012 & \\
\hline
\end{tabular}

\section{References S4}

Abu-Amero, Achilli, A., Olivieri, A., Pala, M., Metspalu, E., Fornarino, S., Battaglia, V. et al. (2007). Mitochondrial DNA Variation of Modern Tuscans Supports the Near Eastern Origin of Etruscans. American Journal of Human Genetics, 80, 759-768. http://dx.doi.org/10.1086/512822 
Álvarez-Iglesias, V., Mosquera-Miguel, A., Cerezo, M., Quintáns, B., Zarrabeitia, M. T., Cuscó, I. et al. (2009). New Population and Phylogenetic Features of the Internal Variation within Mitochondrial DNA Macro-Haplogroup R0. PLoS ONE, 4, e5112. http://dx.doi.org/10.1371/journal.pone.0005112

Brandstatter, A., Klein, R., Duftner, N., Wiegand, P., \& Parson, W. (2006). Application of a Quasi-Median Network Analysis for the Visualization of Character Conflicts to a Population Sample of Mitochondrial DNA Control Region Sequences from Southern Germany (Ulm). International Journal of Legal Medicine, 120, 310-314. http://dx.doi.org/10.1007/s00414-006-0114-x

Cardoso, S., Alfonso-Sanchez, M. A., Valverde, L., Odriozola, A., Perez-Miranda, A. M., Pena, J. A., \& de Pancorbo, M. M. (2011). The Maternal Legacy of Basques in Northern Navarre: New Insights into the Mitochondrial DNA Diversity of the Franco-Cantabrian Area. American Journal of Physical Anthropology, 145, 480-488. http://dx.doi.org/10.1002/ajpa.21532

Comas, D., Plaza, S., Wells, R. S., Yuldaseva, N., Lao, O., Calafell, F., \& Bertranpetit, J. (2004). Admixture, Migrations, and Dispersals in Central Asia: Evidence from Maternal DNA Lineages. European Journal of Human Genetics, 12, 495504. http://dx.doi.org/10.1038/sj.ejhg.5201160

Davidovic, S., Malyarchuk, B., Aleksic, J. M., Derenko, M., Topalovic, V., Litvinov, A. et al. (2015). Mitochondrial DNA Perspective of Serbian Genetic Diversity. American Journal of Physical Anthropology, 156, 449-465. http://dx.doi.org/10.1002/ajpa.22670

Derenko, M., Malyarchuk, B., Grzybowski, T., Denisova, G., Rogalla, U., Perkova, M. et al. (2010). Origin and Post-Glacial Dispersal of Mitochondrial DNA Haplogroups C and D in Northern Asia. PLoS ONE, 5, e15214. http://dx.doi.org/10.1371/journal.pone.0015214

Dulik, M. C., Zhadanov, S. I., Osipova, L. P., Askapuli, A., Gau, L., Gokcumen, O. et al. (2012). Mitochondrial DNA and Y Chromosome Variation Provides Evidence for a Recent Common Ancestry between Native Americans and Indigenous Altaians. American Journal of Human Genetics, 90, 229-246. http://dx.doi.org/10.1016/j.ajhg.2011.12.014

Grzybowski, T., Malyarchuk, B. A., Derenko, M. V., Perkova, M. A., Bednarek, J., \& Wozniak, M. (2007). Complex Interactions of the Eastern and Western Slavic Populations with Other European Groups as Revealed by Mitochondrial DNA Analysis. Forensic Science International: Genetics, 1, 141-147. http://dx.doi.org/10.1016/j.fsigen.2007.01.010

Gubina, M. A., Girgol'kau, L. A., Babenko, V. N., Damba, L. D., Maksimov, V. N., \& Voevoda, M. I. (2013). Mitochondrial DNA Polymorphism in Populations of Aboriginal Residents of the Far East. Russian Journal of Genetics, 49, 751-764. http://dx.doi.org/10.1134/S1022795413070065

Hedman, M., Brandstätter, A., Pimenoff, V., Sistonen, P., Palo, J. U., Parson, W., \& Sajantila, A. (2007). Finnish Mitochondrial DNA HVS-I and HVS-II Population Data. Forensic Science International, 172, 171-178. http://dx.doi.org/10.1016/j.forsciint.2006.09.012

Irwin, J., Egyed, B., Saunier, J., Szamosi, G., O’Callaghan, J., Padar, Z., \& Parsons, T. (2007). Hungarian mtDNA Population Databases from Budapest and the Baranya County Roma. International Journal of Legal Medicine, 121, 377-383.

http://dx.doi.org/10.1007/s00414-006-0128-4

Irwin, J. A., Ikramov, A., Saunier, J., Bodner, M., Amory, S., Rock, A. et al. (2010). The mtDNA Composition of Uzbekistan: A Microcosm of Central Asian Patterns. International Journal of Legal Medicine, 124, 195-204. http://dx.doi.org/10.1007/s00414-009-0406-z

Irwin, J., Saunier, J., Strouss, K., Paintner, C., Diegoli, T., Sturk, K. et al. (2008). Mitochondrial Control Region Sequences from Northern Greece and Greek Cypriots. International Journal of Legal Medicine, 122, 87-89. http://dx.doi.org/10.1007/s00414-007-0173-7

Karachanak, S., Carossa, V., Nesheva, D., Olivieri, A., Pala, M., Hooshiar Kashani, B. et al. (2012). Bulgarians vs the Other European Populations: A Mitochondrial DNA Perspective. International Journal of Legal Medicine, 126, 497-503. http://dx.doi.org/10.1007/s00414-011-0589-y

Kushniarevich, A., Sivitskaya, L., Danilenko, N., Novogrodskii, T., Tsybovsky, I., Kiseleva, A. et al. (2013). Uniparental Genetic Heritage of Belarusians: Encounter of Rare Middle Eastern Matrilineages with a Central European Mitochondrial DNA Pool. PLoS ONE, 8, e66499. http://dx.doi.org/10.1371/journal.pone.0066499

Lehocký, I., Baldovič, M., Kádaši, L., \& Metspalu, E. (2008). A Database of Mitochondrial DNA Hypervariable Regions I and II Sequences of Individuals from Slovakia. Forensic Science International: Genetics, 2, e53-e59. http://dx.doi.org/10.1016/j.fsigen.2007.12.008

Malyarchuk, B. A., Grzybowski, T., Derenko, M. V., Czarny, J., Drobnič, K., \& Miścicka-Śliwka, D. (2003). Mitochondrial DNA Variability in Bosnians and Slovenians. Annals of Human Genetics, 67, 412-425. http://dx.doi.org/10.1046/j.1469-1809.2003.00042.x

Malyarchuk, B. A., Vanecek, T., Perkova, M. A., Derenko, M. V., \& Sip, M. (2006). Mitochondrial DNA Variability in the Czech Population, with Application to the Ethnic History of Slavs. Human Biology, 78, 681-696. http://dx.doi.org/10.1353/hub.2007.0014 
Mielnik-Sikorska, M., Daca, P., Malyarchuk, B., Derenko, M., Skonieczna, K., Perkova, M. et al. (2013). The History of Slavs Inferred from Complete Mitochondrial Genome Sequences. PLoS ONE, 8, e54360. http://dx.doi.org/10.1371/journal.pone.0054360

Mikkelsen, M., Sorensen, E., Rasmussen, E. M., \& Morling, N. (2010). Mitochondrial DNA HV1 and HV2 Variation in Danes. Forensic Science International: Genetics, 4, e87-88. http://dx.doi.org/10.1016/j.fsigen.2009.07.007

Prieto, L., Zimmermann, B., Goios, A., Rodriguez-Monge, A., Paneto, G. G., Alves, C. et al. (2011). The GHEP-EMPOP Collaboration on mtDNA Population Data-A New Resource for Forensic Casework. Forensic Science International: Genetics, 5, 146-151. http://dx.doi.org/10.1016/j.fsigen.2010.10.013

Turchi, C., Buscemi, L., Previdere, C., Grignani, P., Brandstatter, A., Achilli, A. et al. (2008). Italian Mitochondrial DNA Database: Results of a Collaborative Exercise and Proficiency Testing. International Journal of Legal Medicine, 122, 199204. http://dx.doi.org/10.1007/s00414-007-0207-1

Yao, Y. G., Kong, Q. P., Wang, C. Y., Zhu, C. L., \& Zhang, Y. P. (2004). Different Matrilineal Contributions to Genetic Structure of Ethnic Groups in the Silk Road Region in China. Molecular Biology and Evolution, 21, 2265-2280. http://dx.doi.org/10.1093/molbev/msh238

Zgonjanin, D., Veselinovic, I., Kubat, M., Furac, I., Antov, M., Loncar, E., \& Omorjan, R. (2010). Sequence Polymorphism of the Mitochondrial DNA Control Region in the Population of Vojvodina Province, Serbia. Legal Medicine (Tokyo, Japan), 12, 104-107. http://dx.doi.org/10.1016/j.legalmed.2009.10.007

Zheng, H.-X., Yan, S., Qin, Z.-D., \& Jin, L. (2012). MtDNA Analysis of Global Populations Support That Major Population Expansions Began before Neolithic Time. Scientific Reports, 2, 745. http://dx.doi.org/10.1038/srep00745

Zimmermann, B., Brandstatter, A., Duftner, N., Niederwieser, D., Spiroski, M., Arsov, T., \& Parson, W. (2007). Mitochondrial DNA Control Region Population Data from Macedonia. Forensic Science International: Genetics, 1, e4-e9. http://dx.doi.org/10.1016/j.fsigen.2007.03.002 